\title{
CONVEX ENTROPY, HOPF BIFURCATION, AND VISCOUS AND INVISCID SHOCK STABILITY
}

\author{
BLAKE BARKER, HEINRICH FREISTÜHLER, AND KEVIN ZUMBRUN
}

\begin{abstract}
We consider by a combination of analytical and numerical techniques some basic questions regarding the relations between inviscid and viscous stability and existence of a convex entropy. Specifically, for a system possessing a convex entropy, in particular for the equations of gas dynamics with a convex equation of state, we ask: (i) can inviscid instability occur? (ii) can there occur viscous instability not detected by inviscid theory? (iii) can there occur the - necessarily viscouseffect of Hopf bifurcation, or "galloping instability"? and, perhaps most important from a practical point of view, (iv) as shock amplitude is increased from the (stable) weak-amplitude limit, can there occur a first transition from viscous stability to instability that is not detected by inviscid theory? We show that (i) does occur for strictly hyperbolic, genuinely nonlinear gas dynamics with certain convex equations of state. while (ii) and (iii) do occur for an artifically constructed system with convex viscosity-compatible entropy. We do not know of an example for which (iv) occurs, leaving this as a key open question in viscous shock theory, related to the principal eigenvalue property of Sturm Liouville and related operators. In analogy with, and partly proceeding close to, the analysis of Smith on (non-)uniqueness of the Riemann problem, we obtain convenient criteria for shock (in)stability in the form of necessary and sufficient conditions on the equation of state.
\end{abstract}

\section{CONTENTS}

1. Introduction

1.1. Inviscid analysis

1.2. Viscous analysis

1.3. Discussion and open problems

2. Signed Lopatinski criterion and relations to other conditions 8

2.1. The signed Lopatinski determinant 8

2.2. The Lopatinski condition in gas dynamics 10

2.3. Sufficient global conditions for (in)stability 12

2.4. Monotonicity of the Hugoniot curve 13

3. Local conditions for (in)stability and the assumptions of Smith 14

3.1. Local conditions and their requirements 14

3.2. The assumptions of Smith

3.3. General pressure laws

3.4. Link to the Riemann Problem

4. Gas-dynamical examples and counterexamples 20

4.1. Global counterexample $\quad 20$

4.2. Local counterexample 20

4.3. Stable example

5. The Evans function 24

Date: October 15, 2018.

Research of B.B. was partially supported under NSF grant no. DMS-0300487.

Research of H.F. was partially supported under DFG Excellence Grant 2007-2012 to the University of Konstanz. Research of B.B. and K.Z. was partially supported under NSF grant no. DMS-0300487. 
5.1. Viscous vs. inviscid stability 25

5.2. Numerical stability analysis 25

6. Gas dynamical examples $\quad 26$

6.1. Numerical details

6.2. Global counterexample 28

6.3. Local counterexample 29

6.4. Stable example 29

7. Designer systems 32

7.1. The rotating model 32

7.2. Numerical results 34

Appendix A. Lopatinski computations for partial equation of state 38

Appendix B. Helmholtz energy and associated energy functions 39

Appendix C. Numerical Evans function protocol 40

C.1. Profile and Evans function equations 40

C.2. Winding number computations 41

C.3. Hardware and computational statistics 42

References 43

\section{INTRODUCTION}

Stability of shock waves has been the subject of intensive investigation over the more than 50 years since the question was opened in the inviscid setting by Landau, Kontorovich, Dy'akov, Lax, Erpenbeck, and others in the early 1960's. Before that period, shocks seem to have been assumed to be universally stable, and in fact under most reasonable conditions, they are so. See [BE] for an interesting general discusion of these early investigations, and $[\mathrm{S}, \mathrm{MP}]$ on the related question of uniqueness of Riemann solutions; we recommend also the original sources [Er1, Er2, G, M1, M2, M3. Starting in the mid-1980's, these investigations have been widened to include also viscous shock stability, or stability in the presence of regularizing transport effects such as viscosity, heat conduction, magnetic resistivity, and species diffusion. See the surveys [Z1, Z2, Z3, Z5] for general accounts of progress in this direction, and [BiB, GMWZ1, GMWZ2] for accounts of progress on the related inviscid limit problem. See also the recent investigations [TZ1, TZ2, TZ3, SS, BeSZ] on the possibility of Hopf bifurcation of viscous shocks, and stability of resulting time-periodic "galloping" shocks.

At a technical level, stability and bifurcation of shock waves is now fairly well understood, both in one and multi-dimensions. In particular, nonlinear inviscid shock stability reduces [M1] to a spectral condition that may be checked by evaluation of an associated Evans-Lopatinski function commputable by standard linear algebraic operations; see [Er1, M1, T, FP for studies of the Lopatinski function in various contexts. Likewise, determination of nonlinear viscous shock stability/bifurcation has been reduced to verification of spectral conditions on the linearized operator about the wave, that may be readily checked by efficient and well-conditioned numerical Evans function computations as described in [Br, $\mathrm{BrZ}, \mathrm{HuZ1}, \mathrm{Z4}, \mathrm{Z5}$ ] (see, for example, the recent studies [HLZ, BHRZ, BHZ1, BLeZ, BLZ, HLyZ1, HLyZ2, ZZ6). (Cf. [HuZ3, PZ, FS1, FS2, [HLZ, Thm. 1.4], [BHZ1, section 4] for situations in which the spectral conditions may be checked analytically.)

However, at a level of basic intuition/understanding, some important questions remain, even in the one-dimensional setting. In particular, for all of the above-mentioned investigations of physical systems, whether inviscid or viscous, shocks were seen to be one-dimensionally stable. Indeed, as regards physically relevant systems of continuum mechanics, one-dimensionally unstable waves have so far been found only for media permitting phase transitions [GZ, Z6, ZMRS, BM, TZ4], 
and established wisdom [Er1, BE, $\mathrm{MP}$, seems to say that instability is associated with effects not modeled in an ideal gas equation of state.

One could ask, therefore, whether there might be some simple and commonly satisfied structural condition guaranteeing one-dimensional stability. In particular, in the simplest and most familiar setting of inviscid gas dynamics, could the classical condition of thermodynamic stability, or convexity of the equation of state

$$
e=\bar{e}(\tau, S)
$$

relating internal energy e to specific volume $\tau$ and entropy $S$ by itself be sufficient to imply onedimensional stability of shock waves? Remarkably, the answer to this natural question up to now does not appear to have been known.1

A natural generalization pointed out by Lax [L] of the property of thermodynamic stability to arbitrary systems of inviscid conservation laws

$$
u_{t}+f(u)_{x}=0
$$

is existence of a convex entropy, i.e., a function $\eta(u)$ satisfying

$$
d^{2} \eta>0 \quad \text { and } \quad d \eta d f=d q
$$

for an appropriate entropy flux function $q(u)$, whence, for smooth solutions,

$$
\eta(u)_{t}+q(u)_{x}=0 .
$$

One could wonder whether for general systems (1.1), existence of a convex entropy is sufficient to imply one-dimensional stability of inviscid shock waves. This question includes the above one as a special case, as in the case of gas dynamics, one can take $\eta$ as the negative of the thermodynamical entropy $S(\tau, e)$, the Legendre transform of $\bar{e} ?^{2}$

For viscous systems of conservation laws

$$
u_{t}+f(u)_{x}=\left(B(u) u_{x}\right)_{x}
$$

shock waves are heteroclinic traveling waves. It is known [GZ, ZS that viscous stability is a stronger condition than inviscid stability. However, up to now it is not known whether this logical implication of inviscid by viscous stability is strict for any interesting general class of constituents $(f, B)$. Destabilization by viscous effects of an inviscidly stable shock wave would be a physically interesting phenomenon if it occurs, and similar phenomena arising in Orr-Sommerfeld theory and incompressible flow make this not implausible. On the other hand, if this could be shown not to occur, that would be equally interesting, and would greatly simplify the verification of stability, reducing this to the study of the simpler inviscid Lopatinski function, a linear-algebraic quantity, rather than the viscous Evans function, defined in terms of solutions of an associated eigenvalue ODE. In this connection, we were wondering whether the existence of a viscosity-compatible entropy, $\eta$ as above with now also

$$
d^{2} \eta B \geq 0
$$

might imply equivalence of viscous and inviscid instability.

As pointed out in [Z1, Z2, TZ2, TZ3], the same analysis [GZ, ZS] showing that viscous stability implies inviscid stability, shows that, as long as the viscous profile remains transverse as the intersection of the invariant manifolds as which it is defined, viscous destabilization of a stable inviscid shock necessarily must occur through the passage through the imaginary axis into the unstable (positive real part) half plane of a complex conjugate pair of eigenvalues of the linearized operator about the wave, a leading-mode Hopf bifurcation. Here, we are imagining a transition from viscous stability to instability as some bifurcation parameter, typically shock amplitude, is varied.

\footnotetext{
${ }^{1}$ The suggested counterexample of $[\mathrm{G}$ ] is incorrect, as we show below; see Remark 1.4

${ }^{2} S$ is concave if and only if $\bar{e}$ is convex. See [MP] or Lemma 6.7 in [Z1].
} 
"Galloping instabilites" arising through leading-mode Hopf bifurcations are familiar in detonation theory [TZ4], but have up to now not been observed in the shock wave context. Could it be that the existence of a viscosity-compatible entropy precludes complex, or even just purely imaginary eigenvalues? Or could it possibly imply a "[strong] principal eigenvalue property" that any non-stable eigenvalue, of the linearized operator about the wave, with largest possible real part must be real [and simple]? In both cases, leading-mode Hopf bifurcation would be impossible. Could it even be that existence of a viscosity-compatible entropy implied both transversality of the shock profile and impossiblity of leading-mode Hopf bifurcation? In that case, viscous and inviscid stability would coincide.

In this paper, we examine these and related questions using a combination of analytical and numerical techniques. The following are the main results of this examination:

Theorem A (on gas dynamics). (i) There exist equations of state $e=\bar{e}(\tau, S), \tau>0, S \in \mathbb{R}$, that (a) satisfy the standard assumptions (J1)-(J4), (G1) - (G6), and (- 1 1) $-(\mathrm{H} 4)$ detailed below, with in particular, the largest and smallest eigenvalues simple and genuinely nonlinear, entropy and shock speed monotone along the forward 1- and 3-Hugoniot curves, and a global concave entropy function $S(\tau, e)$ defined on $\tau, e>0$, and (b) admit inviscidly unstable shock waves.

(ii) The equation of state

$$
\bar{e}(\tau, S)=\frac{e^{S}}{\tau}+C^{2} e^{S / C^{2}-\tau / C}, \quad C>>1,
$$

is an example for (i).

Numerical Observation A (on gas dynamics). For the equation of state (1.3), in all cases we investigated numerically, (a) viscous [in]stability is equivalent to inviscid [in]stability,

(b) the viscous-stability problem has no non-zero imaginary eigenvalues; in particular, transitions from stability to instability occur exclusively by real eigenvalues passing through the origin, and

(c) in situations of instability, the eigenvalue with largest real part is real and simple.

Numerical Observation B (on general systems). There exist viscous systems (1.2) of conservation laws, endowed with a compatible entropy, that

(a) admit shocks that are inviscidly stable, but viscously unstable,

(b) the viscous-stability problem sometimes does have non-zero imaginary eigenvalues, while

(c) in all situations of instability we investigated numerically, the eigenvalue with largest real part is real, and transitions from stability to instability occur exclusively by real eigenvalues passing through the origin,

(d) in some cases, there are an even number of unstable (and all real) eigenvalues, and

(e) in some cases the eigenvalue with largest real part is not simple.

Our analysis divides roughly into an inviscid and a viscous part, which we now sketch.

1.1. Inviscid analysis. In the first part of the paper, we revisit the inviscid stability analysis of Erpenbeck-Majda [Er1, M1, M2, M3] and reconcile it with the analysis of Smith [S, MP] on nonuniqueness of Riemann solutions, a related but slightly weaker condition than instability of an individual component shock. Let

$$
p=\hat{p}(\tau, e):=-\bar{e}_{\tau}(\tau, \hat{S}(\tau, e))
$$

denote the pressure function determined by inversion of the equation of state $e=\bar{e}(\tau, S)$ for $\tau$ fixed 3

\footnotetext{
${ }^{3}$ A pressure law $p=\hat{p}(\tau, e)$ may also be considered in the absence of an equation of state, being sufficient by itself to close the equations of gas dynamics $\mathrm{MP}, \mathrm{Sm}$. For the observation that any pressure law can to some (interesting!) extent be interpreted as being associated with an equation of state, cf. Sec. 3.3.
} 
Smith formulates criteria on the equation of state, amounting to a weak condition

$$
-\frac{\bar{e}_{\tau s}}{\bar{e}_{S} \bar{e}_{\tau \tau}}<-\frac{2}{\bar{e}_{\tau}}, \text { or, equivalently, } \hat{p}_{\tau}<\frac{p \hat{p}_{e}}{2},
$$

a medium condition,

(Medium $\left._{U}\right)$

$$
-\frac{\bar{e}_{\tau S}}{\bar{e}_{S} \bar{e}_{\tau \tau}}<-\frac{\frac{\bar{e}_{\tau}^{2}}{2 e \bar{e}_{\tau \tau}}+1}{\bar{e}_{\tau}}, \text { or, equivalently, } \hat{p}_{\tau}<\frac{p^{2}}{2 e},
$$

and a strong condition,

$$
-\frac{\bar{e}_{\tau s}}{\bar{e}_{S} \bar{e}_{\tau \tau}}<-\frac{1}{\bar{e}_{\tau}}, \text { or, equivalently, } \hat{p}_{\tau}<0,
$$

and derives in particular that (MediumU $)$ is equivalent under mild additional hypotheses 4 to uniqueness of Riemann solutions.

Recall now some standard properties of a pressure law $p=\hat{p}(\tau, e), \tau, e \in \mathbb{R}^{+}$:

$$
\begin{gathered}
p=\hat{p}(\tau, e)>0 . \quad \text { (Positivity) } \\
\left(\partial_{\tau}-p \partial_{e}\right) \hat{p}<0 . \quad \text { (Hyperbolicity) } \\
\left(\partial_{\tau}-p \partial_{e}\right)^{2} \hat{p}>0 . \quad \text { (Genuine nonlinearity) } \\
\hat{p}_{e}>0 . \quad \text { (Weyl condition) }
\end{gathered}
$$

The following is the technical centerpiece of Part I of this paper.

Theorem 1.1. Under hypotheses (G1)-(G6), (H1) $-(\mathrm{H} 2)$ of Smith (see Sec. 3.2 below) on the equation of state $\bar{e}$, or, more generally, assumptions (J1)-(J4) on the pressure law $\hat{p}$, positivity of the signed Lopatinski determinant (see Sec. 2.1) (for all shocks) is equivalent to

$\left(\right.$ Medium $\left._{S}\right) \quad-\frac{\bar{e}_{\tau S}}{\bar{e}_{S} \bar{e}_{\tau \tau}}<-\frac{-\frac{\bar{e}_{\tau}}{\sqrt{2 e \bar{e}_{\tau \tau}}}+1}{\bar{e}_{\tau}}$, or, equivalently, $\hat{p}_{\tau}<c p / \sqrt{2 e}=p \sqrt{\frac{p \hat{p}_{e}-\hat{p}_{\tau}}{2 e}}$,

while ([Sm]:) uniqueness of Riemann solutions (for any data) is equivalent to MediumU). The four conditions are related by

$$
\text { Strong } \Rightarrow \text { Medium } \mathrm{U} \text { - } \text { Medium } \mathrm{S} \text {. }
$$

In particular, condition (Strong) by itself is sufficient to imply stability of all shocks, while violation of Weak implies existence of unstable ones.

Theorem A is a corollary of Theorem 1.1 and the following finding.

Theorem 1.2. Equation of state (1.3) satisfies (G1)- G6), (H1)- $\mathrm{H} 2$, and violates Weak).

Both the particular implication (Medium $) \Rightarrow\left(\right.$ Medium $\left._{S}\right)$ in Theorem 1.1 and the general meaning of the signed Lopatinski determinant are elucidated by the following fact, which holds for arbitrary systems.

Theorem 1.3. If the signed Lopatinski determinant $\Delta(\alpha)$ of a family $\left(U_{-}(\alpha), U_{+}(\alpha)\right), \alpha \in \mathbb{R}$ of Lax shocks undergoes a sign change at some critcal value $\alpha_{*} \in \mathbb{R}$, the Riemann problem loses uniqueness near the initial data $\left(U_{l}, U_{r}\right)=\left(U_{-}\left(\alpha_{*}\right), U_{+}\left(\alpha_{*}\right)\right)$.

\footnotetext{
${ }^{4} \mathrm{Cf}$. Theorem 1.1, within which we include Smith's result for the reader's ease.
} 
Remark 1.4. A general polytropic equation of state $\bar{e}(\tau, S)=e^{S / c_{v}} / \tau^{\Gamma}, \Gamma, c_{v}>0$, satisfies (Strong), or $\Gamma^{2}<\Gamma(\Gamma+1)$. By Theorem 1.1, we see that a local counterexample $\bar{e}(\tau, S)=e^{S} / \tau+f(S)$, $f^{\prime} \gg 1$, proposed by C. Gardner (final paragraph of [G]) is incorrect, as this equation of state likewise satisfies (Strong), by essentially the same computation. Nonetheless, Gardner's larger assertion, that "... all of the situations we have considered are logically possible, without violating the fundamental condition for local thermodynamic stability, namely that $e$ is a convex function of $\tau$ and $S$," turns out to be correct, as shown by example (1.3).

Remark 1.5. Taylor expanding $(1.3)$ in $C$, we obtain also a simplified local example

$$
\bar{e}(\tau, S)=e^{S} / \tau+S-C \tau+\tau^{2} / 2, \quad C \gg>1,
$$

of a convex equation of state permitting instabilities, for which Hugoniot curves, monotonicity of $S$ and $\sigma$, etc. may be computed explicitly, giving a concrete illustration of the theory. This does not satisfy (J1), but may be treated by similar techniques as used to establish Theorem A; see Proposition 4.1 below. On the other hand, we find that the seemingly similar example

$$
\bar{e}(\tau, S)=e^{S} / \tau-C \tau+\tau^{2} / 2, \quad C>>1,
$$

does not permit instabilities, despite violating (Weak); see Proposition 4.3 .

Remark 1.6. Example (1.3) is the more surprising in view of recent results [LV] in nearby settings showing that existence of a convex entropy implies stability. Indeed, Theorem A appears at first sight to contradict Theorem 2 of [LV], which asserts that, under some mild technical assumptions, existence of a convex entropy, simplicity of extremal characteristics, and monotonicity of entropy and shock speed along forward Hugoniot curves together imply inviscid stability of arbitrary-amplitude extremal (i.e., 1- or 3-) shocks. This would be very interesting to resolve; at the least, it shows that there is a very narrow window between negative and positive results.

Remark 1.7. Smith's weak and strong conditions are phrased in terms of the gas law $e=\check{e}(\tau, p)$ obtained by inverting $p=\hat{p}(\tau, e)$ with respect to $e$, under the assumption that $\hat{p}_{e}>0$ ((J4) below). Our versions are equivalent to those of Smith if and only if $\hat{p}_{e}>0$, through the relation $\check{e}_{\tau}=-\frac{\hat{p}_{\tau}}{\hat{p}_{e}}$, but remain valid also in the general case, as Smith's therefore do not. Similar observations are made in [MP] regarding the logical ordering of Smith's conditions, which again requires $\hat{p}_{e}>0$. Thus, our conditions above are in fact extensions of Smith's conditions into the realm $\hat{p}_{e} \leq 0$, or, equivalently (by $\left.\bar{p}_{S}=\hat{p}_{e}(d e / d S)=\hat{p}_{e} T\right), \bar{p}_{S} \leq 0$. In this case, other aspects of Smith's global theory break down, in particular, the conclusions of Proposition 3.2 and, thus, Theorem 1.1. However, we find that individual 1-shocks $\left(U_{-}, U_{+}\right)$are stable for $\left.\hat{p}_{e}\right|_{U_{+}} \leq 0$; see Corollary 2.6.

1.2. Viscous analysis. In the remainder of the paper, we investigate viscous stability of the above and other systems via numerical Evans function computations, seeking viscous instabilities not predicted by inviscid theory, and in particular purely imaginary eigenvalues of the linearized operator about the wave. The Evans function, as described for example in [AGJ, PW, GZ, is an analytic function $D(\lambda)$ associated with a viscous shock wave, defined on the nonstable complex half-plane $\{\lambda: \Re \lambda \geq 0\}$, whose zeros encode the stability properties of the wave, in particular, corresponding away from the origin with eigenvalues of the linearized operator about the wave. See [Z1, [Z2, Z3, [Z5, TZ2, TZ3, [SS, BeSZ] and references therein for more detailed discussions of the relation between the Evans function, spectral, linearized, and nonlinear shock stability, and Hopf bifurcation.

As described in [BrZ, HuZ1, Z4, ZZ], the Evans function may be efficiently computed by shooting methods as a Wronskian evaluated at $x=0$ of decaying modes at $x= \pm \infty$ of the eigenvalue ordinary differential equation (ODE) associated with the linearized operator about the wave, and this has by now been carried out successfully for a number of interesting systems/waves arising in 
continuum mechanics using the standard Runga Kutta 4-5 (ode45) routine supported in MATLAB in conjunction with the exterior product or polar coordinate algorithms [Br, $\mathrm{BrZ}$, $\mathrm{HuZ1}$, of the MATLAB-based Evans function package STABLAB [BHZ2], in particular for the computationally intensive problem of stability of detonation waves [HuZ2, BZ1, BZ2, BHLyZ2]).

However, the equations of state (1.3) and (1.4), due to the separation of scales introduced by the large parameter $C \gg 1$ and exponential dependence on parameters, induce a computational difficulty far beyond any of the systems so far considered. In particular, computations with RK45 were not practically feasible, even on a parallel supercomputing machine. To carry out Evans function computations for this system, rather, we found it necessary for the first time to use an ODE solver designed for stiff systems, namely, the ode15s routine supported in MATLAB, a recently-developed algorithm based on modern numerical differentiation formula (NDF) methods.

To indicate the relative stiffness of the systems associated with $(1.3)-(1.4)$ as compared to that of previously considered cases, the performance of Evans computations with ode 45 and ode15s are similar for all previously computed examples; however, in the present case, ode15s outperforms ode45 by 2-3 orders of magnitude, yielding performance that is not only feasible, but in the general range seen for nonstiff computations.

The results of these numerical computations, gathered above as "Numerical Observation A (on gas dynamics)" hold also for equation of state (1.4.

The other results, presented above as "Numerical Observation B (on general systems)", are obtained by computations on an artificially constructed $3 \times 3$ system of viscous conservation laws (1.2) ${ }^{5}$ of a form suggested by Stefano Bianchini [Bi]. Specifically, as shock amplitude is increased, several eigenvalues cross the origin into the unstable half-plane, some of them eventually coalescing, splitting off of the real axis as a complex conjugate pair, and crossing back into the stable halfplane through the imaginary axis at other points than 0 . This is a Hopf bifurcation, though not a leading-mode one. Note finally that the examples with an even number of unstable eigenvalues exemplify a scenario not detected even by signed versions of the inviscid stability condition, - i.e., essentially, the signed Lopatinski determinant - that find the parity of the number of unstable roots as described in [GZ, Z1, Z2].

1.3. Discussion and open problems. In conclusion, we have confirmed the claim of C. Gardner G] made almost 50 years ago, but since then apparently not paid much attention to, that the equations of gas dynamics, even under all of the usual thermodynamical and structural assumptions associated with a "normal" gas, can support unstable inviscid shock waves. Moreover, we have shown this in a somewhat stronger sense than that apparently envisioned by C. Gardner, demonstrating that this can be accomplished not only locally, but globally, with a convex equation of state $e=$ $\bar{e}(\tau, S)$ satisfying expected asymptotics as $S \rightarrow \pm \infty$ or $\tau \rightarrow 0,+\infty$. In the process, we shed new light on the important work of R. Smith [S] and Menikoff-Plohr [MP] on uniqueness of Riemann solutions.

This in a sense validates the inviscid stability theory of Erpenbeck-Majda, showing that stability does not hold "automatically" for equations of state satisfying all conditions of a classical gas. On the other hand, we find so far no distinction between viscous and inviscid stability for shock waves in gas dynamics. We do, however, show by example that such a distinction, and in particular the new phenomenon of Hopf bifurcaton, can occur for a $3 \times 3$ system with global convex entropy. Whether such phenomena arise also for Lax shocks of physical systems such as gas dynamics, magnetohydrodynamics (MHD), or viscoelasticity, must be left to further investigation. We point in particular to the viscous-stability analysis of full (nonisentropic) MHD, so far not carried out even for the classical polytropic equation of state, as a promising candidate and an important problem for further study.

\footnotetext{
5 The same size as the equations of gas dynamics.
} 
Regarding the initial transition from stability to instability, we find no distinction between viscous and inviscid behavior for any of the systems we consider. Likewise, we find no counterexample to the (weak) "principal eigenvalue property" that the largest unstable eigenvalue of the linearized operator about the wave be real. It would seem most interesting to know whether this property holds in any generality.

Finally, recalling that shock instability in the physical literature has often been associated with the phenomenon of phase transition [Er1, BE, $\mathrm{MP}$, we repeat that our examples here do not involve phase transition; rather, the principal mechanism for instability, at a technical level, i.e., the feature leading to violation of (Weak), is stiffness, or appearance of multiple scales via the parameter $C \gg 1$. Indeed, computing $p(\tau, S)=-\bar{e}_{\tau}(\tau, S)=\frac{e^{S}}{\tau^{2}}+C-\tau$ for example (1.4) reveals a similarity to a "stiffened" or "prestressed" equation of state sometimes used to model shock behavior near the liquid state. For example, water is often described by

$$
p=\Gamma \rho e-\gamma P_{0}
$$

with $\Gamma$ and the base stress $P_{0}$ determined empirically [IT, $\left.\mathrm{HVPM}\right]$; similar techniques are used to model liquid argon, nickel, mercury, etc. [H, CDM]. The careful numerical study and categorizaion of behavior of these and other more exotic equations of state such as van der Waals, Redlich-Kwong, etc., both with and without viscosity, appears to be another important direction for further study, and one in which surprisingly little has so far been done. In particular, a viscous counterpart of the inviscid analyses of stability carried out for van der Waals gas dynamics in [ZMRS, BM] would be a valuable addition to the literature.

\section{PART I. INVISCID STABILITY FOR GAS DYNAMICS}

Sections 2 and 3, the first two of the three sections of this first principal part of the paper are devoted to establishing a network of algebraic and geometric conditions on both individual shock waves and Hugoniot curves that alone or together characterize (un)stable shock waves or imply or preclude the existence of such waves in the sense of necessary or sufficient conditions. Part I culminates in Section 4 that shows the existence of inviscidly unstable shock waves in gas dynamics with certain equations of state.

\section{Signed Lopatinski CRiterion AND RELATIONS TO OTHER CONDITIONS}

In this section, we define the signed Lopatinski criterion, evaluate it for gas dynamics with very general equations of state, and characterize its connections with other conditions on the equation of state.

2.1. The signed Lopatinski determinant. We begin by recalling the Lopatinski condition of Erpenbeck-Majda [Er1, M1, M2, M3 for stability of inviscid shock waves. Consider a general system of conservation laws

$$
f^{0}(w)_{t}+f(w)_{x}=0, \quad w \in \mathbb{R}^{n}
$$

and a Lax p-shock [Sm, Se2, Se3] $U_{ \pm}$with speed $\sigma$, satisfying the Rankine-Hugoniot conditions

$$
\sigma\left[f^{0}\right]=[f]
$$

hyperbolicity

$$
\sigma\left(A_{ \pm}\right) \text {real, } \quad A_{ \pm}:=\left(f_{w}^{0}\right)^{-1} f_{w}\left(U_{ \pm}\right),
$$

of endstates $U_{ \pm}$and the Lax conditions

$$
\begin{gathered}
a_{1}^{-} \leq \cdots \leq a_{p-1}^{-}<\sigma<a_{p}^{-}<\cdots \leq a_{n}^{-} \\
a_{1}^{+} \leq \cdots<a_{p}^{+}<\sigma<a_{p+1}^{+} \leq \cdots \leq a_{n}^{+},
\end{gathered}
$$


where $a_{j}^{ \pm}$are the (ordered) eigenvalues of $A_{ \pm}:=\left(f_{w}^{0}\right)^{-1} f_{w}\left(U_{ \pm}\right)$.

In this setting, one-dimensional linearized and (under additional mild structural assumptions) nonlinear inviscid stability is addressed (see [M1, MZ1, MZ2, BS]) by the Lopatinski condition

$$
\delta:=\delta\left(U_{-}, U_{+}\right):=\operatorname{det}\left(A_{-}^{0} r_{1}^{-}, \ldots, A_{-}^{0} r_{p-1}^{-},\left[f^{0}\right], A_{+}^{0} r_{p+1}^{+}, \ldots, A_{+}^{0} r_{n}^{+}\right) \neq 0,
$$

where $r_{j}^{-}, j=1, \ldots, p-1$ are the "outgoing" modes of $A_{-}:=\left(f_{-}^{0}\right)^{-1} f_{w}\left(U_{-}\right)$relative to the shock, i. e., a basis of the invariant space associated with eigenvalues $a_{j}^{-}$such that $a_{j}^{-}-\sigma<0$ and $r_{j}^{+}$, $j=p+1, \ldots, n$ are the "outgoing" modes of $A_{+}:=\left(f_{w}^{0}\right)^{-1} f_{w}\left(U_{+}\right)$, relative to the shock, i. e., a basis of the invariant space associated with eigenvalues $a_{j}^{+}$such that $a_{j}^{+}-\sigma>0$, and $A_{ \pm}^{0}:=f_{w}^{0}$. Implicit in this description is the defining property of a Lax $p$-shock that the outgoing eigenvectors on the left (right) indeed number $p-1(n-p)$.

Remark 2.1. Note that we have allowed here by the inclusion of $f^{0}$ a somewhat more general formulation than in (1.1). In the simplest case $f^{0}(w)=w$ occurring in many applications, the computations considerably simplify. However, as we shall see in the full gas case, there can in some cases be an advantage in being able to choose coordinates more flexibly. A short proof of the case $f(w)=w$ may be found in [ZS]. Redefining $\tilde{w}:=f^{0}$, we obtain $\tilde{A}_{+}=f_{\tilde{w}}=f_{u}\left(f_{u}^{0}\right)^{-1}$, yielding the result by the observation that eigenvectors $\tilde{r}_{j}^{+}$of $\tilde{A}_{+}$are equal to $A_{+}^{0} r_{j}^{+}$. See also [Se2, Se3, M1].

Definition 2.2. Consider a given p-shock $\left(U_{-}, U_{+}\right)$for a system (2.1). Assume that there is a continuous one-parameter family $\left(U^{-}(\alpha), U^{+}(\alpha)\right), 0<\alpha \leq 1$, of p-shocks with $U^{ \pm}(1)=U_{ \pm}$and $U^{ \pm}(0+)=U_{*}$ for some state $U_{*}$, that the bases $\left\{r_{1}^{-}, \ldots, r_{p-1}^{-}\right\},\left\{r_{p+1}^{+}, \ldots, r_{n}^{+}\right\}$and thus

$$
\delta(\alpha):=\delta\left(U^{-}(\alpha), U^{+}(\alpha)\right)
$$

are (chosen) continuous in $\alpha$, and that the expression

$$
\left.\Delta \equiv \delta(1) / \lim _{\alpha \searrow 0} \operatorname{sgn} \delta(\alpha)\right),
$$

makes sense and has the same sign for all such homotopies. Then $\Delta=\Delta\left(U_{-}, U_{+}\right)$is called a signed Lopatinski determinant of $\left(U_{-}, U_{+}\right)$.

Obviously, the signed Lopatinski determinant $\Delta$ is well defined ( - more precisely, while the Lopatinski determinant $\delta$ is defined up to a non-vanishing factor, the signed Lopatinski determinant $\Delta$ is defined up to a positive factor - ) at least whenever

the system's state space is simply connected,

$a_{p}$ is an everywhere simple, genuinely nonlinear eigenvalue, and

all $p$-shocks with arbitrary fixed left state $U_{-}$group as regular directed curves $S_{p}\left(U_{-}\right)$.

In the current Part I of this paper we use the signed Lopatinski determinant to detect instability according to the following evident fact.

Corollary 2.3. If a given hyperbolic system (2.1) satisfying 2.6) admits a p-shock with $\Delta<0$, then it also admits a p-shock with $\delta=0$.

Proof. For a corresponding homotopy, obviously

$$
\Delta\left(U^{-}(\alpha), U^{+}(\alpha)\right)>0 \quad \text { for sufficiently small } \alpha>0,
$$

and the existence of an $\alpha_{*}$ with $\Delta\left(U^{-}\left(\alpha_{*}\right), U^{+}\left(\alpha_{*}\right)\right)=0$ follows by continuity.

For the (deeper) meaning of the signed Lopatinski determinant in the viscous context, see Part II, Sec. 6. 
Remark 2.4. As noted in [Fo] in the context of gas dynamics, and [Se1 more generally, a change in the sign of $\Delta$ may also be interpreted in terms of multi-dimensional inviscid theory, signalling a transition from weak multi-dimensional stability in the sense of Majda [M1, M2, M3] to exponential instability; see also [MP, Z1, Z3].

2.2. The Lopatinski condition in gas dynamics. Specializing to gas dynamics, we now compute the Lopatinski determinant and signed Lopatinski determinant explicitly.

Isentropic gas dynamics. The isentropic Euler equations in Lagrangian coordinates are

$$
\begin{aligned}
& \tau_{t}-v_{x}=0, \\
& v_{t}+p_{x}=0,
\end{aligned}
$$

with a given pressure law $p=p(\tau)$, where $\tau$ denotes specific volume, $v$ velocity, and $p$ pressure. From $A_{ \pm}=\left(\begin{array}{cc}0 & -1 \\ p_{\tau} & 0\end{array}\right)_{ \pm}$, we readily find that $a_{j}^{ \pm}=-c, c, c:=\sqrt{-p^{\prime}(\tau)}$, and $r_{j}^{ \pm}=\left(\begin{array}{c}1 \\ -a_{j}^{ \pm}\end{array}\right)$, so that hyperbolicity corresponds to $p^{\prime}<0$.

Moreover, $(\mathrm{RH})$ gives $\sigma[\tau]=-[v], \sigma[v]=[p]$, yielding $\sigma^{2}=-[p] /[\tau]$, whence, for a 1-shock, $\sigma=-\sqrt{-[p] /[\tau]}$, and so we have $\left[f^{0}\right]=[\tau]\left(\frac{1}{\sqrt{-[p] /[\tau]}}\right)$. Thus,

$$
\delta=[\tau] \operatorname{det}\left(\begin{array}{cc}
1 & 1 \\
-\sigma & -c
\end{array}\right)=[\tau](\sigma-c)<0 \quad \text { and } \quad \Delta>0
$$

for all Lax shocks. Notice that condition (2.6) is satisfied trivially. Note that $[\tau] \neq 0$, since otherwise $[v]=0$ by $(\mathrm{RH})$, and there is no shock.

Full gas dynamics. In Lagrangian coordinates, the full (nonisentropic) Euler equations are

$$
\begin{aligned}
\tau_{t}-v_{x} & =0, \\
v_{t}+p_{x} & =0, \\
\left(e+v^{2} / 2\right)_{t}+(v p)_{x} & =0,
\end{aligned}
$$

with a given pressure law $p=\hat{p}(\tau, e)$, where $\tau$ denotes specific volume, $v$ velocity, $e$ specific internal energy, and $p$ pressure. We assume here that the pressure law originates from a thermodynamic equation of state

$$
e=\bar{e}(\tau, S),
$$

where $S$ is entropy, through the relation $p=-\bar{e}_{\tau}$, assuming that temperature $T=\bar{e}_{S}$ is positive, so that 2.9 may be solved for $S=\hat{S}(\tau, e)$ to obtain $\hat{p}(\tau, e)=\bar{e}(\tau, \hat{S}(\tau, e))$.

Alternatively, for smooth solutions, we have the simple entropy form $[\mathrm{Sm}$ ]

$$
\begin{aligned}
\tau_{t}-v_{x} & =0, \\
v_{t}+p_{x} & =0, \\
S_{t} & =0, .
\end{aligned}
$$

where $p=p(\tau, S)=-\bar{e}_{\tau}(\tau, S)$ for equation of state 2.9$)$. Choosing $w=(\tau, v, S)^{T}$, we thus have

$$
A_{ \pm}=\left(\begin{array}{ccc}
0 & -1 & 0 \\
p_{\tau} & 0 & p_{S} \\
0 & 0 & 0
\end{array}\right)_{ \pm}
$$

and, essentially by inspection,

$$
a_{1}=-c, a_{2}=0, a_{3}=c, \quad c:=\sqrt{-p_{\tau}},
$$


and $r_{1}^{+}=\left(\begin{array}{l}1 \\ c \\ 0\end{array}\right), r_{2}^{+}=\left(\begin{array}{c}-p_{S} \\ 0 \\ p_{\tau}\end{array}\right), r_{3}^{+}=\left(\begin{array}{c}1 \\ -c \\ 0\end{array}\right)$, so that hyperbolicity corresponds to $p_{\tau}=-\bar{e}_{\tau \tau}<0$. Computing $A_{ \pm}^{0}=\left(\begin{array}{ccc}1 & 0 & 0 \\ 0 & 1 & 0 \\ \bar{e}_{\tau} & v & \bar{e}_{S}\end{array}\right)_{ \pm}=\left(\begin{array}{ccc}1 & 0 & 0 \\ 0 & 1 & 0 \\ -p & v & T\end{array}\right)_{ \pm}$, we obtain

$$
A_{+}^{0} r_{1}^{+}=\left(\begin{array}{c}
1 \\
c \\
-p+v c
\end{array}\right), \quad A_{+}^{0} r_{2}^{+}=\left(\begin{array}{c}
-p_{S} \\
0 \\
p p_{S}+T p_{\tau}
\end{array}\right), \quad A_{+}^{0} r_{3}^{+}=\left(\begin{array}{c}
1 \\
-c \\
-p-v c
\end{array}\right) .
$$

Next, from $(\overline{\mathrm{RH}})$, we have, taking without loss of generality $v_{-}=0$,

$$
[v]=-\sigma[\tau], \quad\left[e+v^{2} / 2\right]=\sigma^{-1}[v p]=-p_{+}[\tau], \quad \sigma^{2}=-[p] /[\tau],
$$

so that, for a 1-shock (recalling the relation $\left.p_{\tau}=-c^{2}\right), \delta=[\tau] \operatorname{det}\left(\begin{array}{ccc}1 & -p_{S} & 1 \\ -\sigma & 0 & -c \\ -p & p p_{S}-T c^{2} & -p-v c\end{array}\right)$, or

$$
\delta=[\tau]\left(p_{S} c[p]+T c^{2}(\sigma-c)\right),
$$

where all quantities are evaluated at $U_{+}$, and $c:=\sqrt{-p_{\tau}}$ as above denotes sound speed. Note that $[\tau] \neq 0$, or else $[v]=0,[p]=0$, and thus $[e]=0$, all by $(\overline{\mathrm{RH}})$. But, $[e]=[\tau]=0$ and $[\tau]=0$ by $\bar{e}_{S}>0$ implies that $[S]=0$ and there is no shock.

Note that, in the decoupled case $p_{S}=0$, we get $\delta=[\tau]\left(T c^{2}(\sigma-c)\right)<0$, by $T>0$, essentially reducing to the isentropic case.

We assume, here and henceforth, that condition (2.6) holds for the Euler equations (2.8). Note that whenever Smith's conditions (G1)-G6), (H1)-(H2) (cf. Sec. 3.2) hold, this is automatic.

Theorem 2.5. For equations 2.8 with $p_{\tau}<0$ a Lax 1-shock satisfies the signed Lopatinski condition $\Delta>0$ if and only if

$$
p_{S}[p]<T p_{\tau}(\sigma / c-1)
$$

or, alternatively,

$\left(\right.$ Lop $\left._{\text {alt }}\right)$

$$
\hat{p}_{\tau}[p]<-c \sigma,
$$

where all quantities not in [.] are evaluated at $U_{+}$.

Proof. By homotopy from the general case to the decoupled case. Equivalence of (Lop alt and (Lop follows by $p_{\tau}=-c^{2}=\hat{p}_{\tau}-p \hat{p}_{e}$ and $\hat{p}_{e}(\tau, e)=\bar{p}_{S}(\tau, S) / \bar{e}_{S}(\tau, S)$.

We state the following elementary result mainly in order to illustrate the competition between isentropic and nonisentropic effects.

Corollary 2.6. For a gas with equation of state satisfying $T, p>0, p_{\tau}<0$, and ("anti-Weyl" condition) $\left.p_{S}\right|_{U_{+}} \leq 0$, all Lax shocks satisfy the signed Lopatinski condition $\Delta>0$.

Proof. In this case, each of the terms $p_{S} c[p]$ and $-T p_{\tau}(\sigma-c)$ on the righthand side of (2.13) are strictly negative, by $T>0$ and $p_{\tau}<0$ (hyperbolicity), so that $\operatorname{sgn} \delta=\operatorname{sgn}[\tau]$. But $[\tau]<0$ for any 1-shock and $[\tau]>0$ for any 3 -shock.

Remark 2.7. Though we have restricted for clarity to the case that the pressure relation derives from a complete equation of state $e=\bar{e}(\tau, S)$, we could equally well carry out the analysis within the framework (2.8), with $p=\hat{p}(\tau, e)$, to obtain the condition $\hat{p}_{e}[p]<c^{2}(1-\sigma / c)$ or, more simply, (Lopalt); see Appendix A. This could alternatively be obtained indirectly, by the observation that, 
for any choice of positive temperature function $T=T(\tau, e)$ defined in the vicinity of $U_{+}$, we can invert the Thermodynamic relation $d e=T d S-p d \tau$ to obtain a local entropy $S$ and equation of state $e=\bar{e}(\tau, S)$; see Section 3.3 .

Remark 2.8. Though the pressure law $p=\hat{p}(\tau, e)$ is sufficient to close the Euler equations, the Navier-Stokes equations, involving the Fourier law, require also the specification of a temperature law $T=\hat{T}(\tau, e)$. Both can be obtained from an equation of state $e=\bar{e}(\tau, S)$, as described above.

Relation to Majda's condition. We now verify that, assuming the Weyl condition $p_{S}>0$, our condition (Lop) agrees with Majda's 1D stability condition (more precisely, the signed version obtained by taking into account multi-dimensiional considerations; see Remark B.6, p. 523-524, Appendix C, [Z1]):

$$
\frac{1+M}{\Gamma}>\left(\frac{\tau_{-}}{\tau}-1\right) M^{2}
$$

where $\Gamma$ is the Gruneisen coefficient $\Gamma:=\frac{\tau p_{S}}{T}$, and $M^{2}=\frac{\left(v-\sigma_{E}\right)^{2}}{c_{E}^{2}}$, the subscript $E$ denoting Eulerian values. Evidently, $\Gamma>0$ if and only if $p_{S}>0$.

Rewriting 2.14 as $\frac{\Gamma}{1+M}<\frac{\tau}{-[\tau] M^{2}}$ using positivity of $\Gamma$ and substituting $\frac{\tau p_{S}}{T}$ for $\Gamma$, we have

$$
\frac{-[\tau] p_{S}}{T}<\frac{1+M}{M^{2}} .
$$

We may compare to our condition (Lop) by expressing the (Eulerian) Mach number $M$ in terms of Lagrangian quantities. Evidently, we must verify the identity $\frac{T}{-[\tau]} \frac{1+M}{M^{2}}=\frac{T p_{\tau}(\sigma-c)}{c[p]}$, or

$$
\frac{1+M}{M^{2}}=\frac{c(c-\sigma)}{\sigma^{2}} .
$$

In Eulerian coordinates, we have $\sigma_{E}[\rho]=[\rho v]$, or $\sigma_{E}=\frac{v / \tau}{[1 / \tau]}=\frac{-v}{[\tau]}=\sigma$, in agreement with the Lagrangian value. Moreover, $v=[v]=-\sigma[\tau]$ gives $v-\sigma=-\sigma([\tau]+1)$, so that, in case $\tau_{0}=1$, we have $v-\sigma=-\sigma \tau$. On the other hand, $c_{E}=\tau c$, hence $M=\frac{v-\sigma}{\tau c}$, and the desired identity is $\frac{1+(v-\sigma) / \tau c}{(v-\sigma)^{2} /(\tau c)^{2}}=\frac{c(c-\sigma)}{\sigma^{2}}$, or $\frac{\tau c+(v-\sigma)}{(v-\sigma)^{2}}=\frac{(c-\sigma)}{\tau \sigma^{2}}$. Without loss of generality (by rescaling) taking $\tau_{-}=1$, $v-\sigma=-\sigma \tau$, this becomes $\frac{\tau c-\sigma \tau}{\sigma^{2} \tau^{2}}=\frac{(c-\sigma)}{\tau \sigma^{2}}$, which is evidently true.

Remark 2.9. Similarly as in Remark 1.7, we find that our condition (Lop) is an extension of Majda's condition into the realm $\left.p_{S}\right|_{U_{+}} \leq 0$ where Majda's condition no longer applies. Indeed, for this case $\Gamma \leq 0$ and (2.14) fails always, whereas we see by (Lop) that stability in fact always holds.

2.3. Sufficient global conditions for (in)stability. The following sufficient conditions for satisfaction and violation, respectively, of the signed Lopatinski condition are as easy to get as useful. For definiteness, we restrict to the case of 1-shocks.

Proposition 2.10. For a Lax 1-shock with $[\tau]<0$, the signed Lopatinski condition $\Delta>0$ is implied by

(Strong')

$$
-\left.\frac{\bar{e}_{\tau S}}{\bar{e}_{S} \bar{e}_{\tau \tau}}\right|_{U=U_{+}}<\frac{1}{[p]} .
$$

The opposite, $\Delta<0$, is implied by failure of

$($ Weak')

$$
-\left.\frac{\bar{e}_{\tau S}}{\bar{e}_{S} \bar{e}_{\tau \tau}}\right|_{U=U_{+}}<\frac{2}{[p]} .
$$


Proof. Because of $[\tau]<0,[p]$ is positive, and dividing both sides of (Lop) by the positive quantity $c[p]$, we obtain

$$
-\frac{\bar{e}_{\tau S}}{\bar{e}_{S} \bar{e}_{\tau \tau}}<\frac{-\frac{\sigma}{c}+1}{[p]}=\frac{\frac{|\sigma|}{c}+1}{[p]} .
$$

Recalling, 2.11), that the characteristic speeds for (2.8)-2.10 are $-c, 0, c$, we have for a Lax 1 -shock that at the right state $U_{+},-c<\sigma<0<c$, or $\sigma<0$ and $|\sigma|<c$. Hence, $1<-\frac{\sigma}{c}+1<2$, from which we obtain with $\left(\mathrm{Lop}_{1}\right)$, the stated stability conditions.

Remark 2.11. Conditions (Strong') and (Weak') are global versions of conditions (Weak and Strong of $[\underline{S}$. Evidently, (Weak $\Rightarrow$ Weak') and (Strong $\Rightarrow$ Strong').

2.4. Monotonicity of the Hugoniot curve. Using the formulation (Lop), we show how stability of shocks relates to geometric properties of the Hugoniot curve, recovering both the classical observations of Erpenbeck and Gardner [Er1, G] relating stability to geometric properties of the Hugoniot curve and the conditions of Smith for uniqueness of Riemann solutions. It remains to investigate monotonicity of the 1- (or, equivalently, the 3-) Hugoniot curve. From the assumption $\bar{e}_{S}=T>0$, we may solve $e=\bar{e}(\tau, S)$ to obtain $S=\hat{s}(\tau, e)$, and thereby

$$
p=\hat{p}(\tau, e):=-\bar{e}_{\tau}(\tau, \hat{s}(\tau, e)) .
$$

Combining the three equations $(\overline{\mathrm{RH}})$, we readily obtain the single equation

$$
\hat{H}(\tau, e)=[e]+(1 / 2)\left(p+p_{-}\right)[\tau]=0
$$

for $(e, \tau)=\left(e_{+}, \tau_{+}\right)$, implicitly determining, together with (2.15), $e$ as a function of $\tau$ or vice versa. Alternatively, using (2.12) to relate different variables, we could consider this as determining $p$ as a function of $v$, or $\tau$ as a function of $s$, whose derivatives in each case may be computed by a straightforward application of the Implicit Function Theorem. We record the results here.

Lemma 2.12. We have the change of variables formulae

$$
\hat{s}_{e}=\frac{1}{\bar{e}_{s}}, \quad \hat{s}_{\tau}=-\frac{\bar{e}_{\tau}}{\bar{e}_{s}}, \quad \hat{p}_{\tau}=-\bar{e}_{\tau \tau}-\bar{e}_{\tau s} \hat{s}_{\tau}=-\bar{e}_{\tau \tau}+\frac{\bar{e}_{\tau s} \bar{e}_{\tau}}{\bar{e}_{s}}, \quad \hat{p}_{e}=-\bar{e}_{\tau s} \hat{s}_{e}=-\frac{\bar{e}_{\tau s}}{\bar{e}_{s}},
$$

and, along the Hugoniot curve $H \equiv 0$, viewing e and $p$ as functions $e=e_{H}(\tau)$ and $p=p_{H}(\tau)$,

$$
\frac{d e_{H}}{d \tau}=\frac{-\frac{1}{2}\left(\hat{p}_{\tau}[\tau]+p+p_{-}\right)}{1+\frac{1}{2} \hat{p}_{e}[\tau]}, \quad \frac{d p_{H}}{d \tau}=\hat{p}_{\tau}+\hat{p}_{e} \frac{d e_{H}}{d \tau}=\frac{\frac{1}{2}[p] \hat{p}_{e}-c^{2}}{1+\frac{1}{2} \hat{p}_{e}[\tau]} .
$$

Alternatively, considering $v$ as a function of $p$ along $H \equiv 0$, we have, taking $v_{-}=0$,

$$
-2 v \frac{d v}{d p}=[\tau] \frac{[p] \hat{p}_{e}-c^{2}-\sigma^{2}}{\frac{1}{2}[p] \hat{p}_{e}-c^{2}} .
$$

Proof. Relations (2.17) and 2.18) follow directly from the Implicit Function Theorem applied to 2.15 and 2.16), where the final equality in 2.18) follows from $\frac{d p_{H}}{d \tau}=\hat{p}_{\tau}+\hat{p}_{e} \frac{d e_{H}}{d \tau}=\frac{-\frac{1}{2}\left(p+p_{-}\right) \hat{p}_{e}+\hat{p}_{\tau}}{1+\frac{1}{2} \hat{p}_{e}[\tau]}$ together with $c^{2}=-\hat{p}_{\tau}+p \hat{p}_{e}$. From $v=-\sqrt{-[p][\tau]}$ (a consequence of $\left(\mathrm{RH}\right.$ ) and $v_{-}=0$, through $[v]=-\sigma[\tau])$, we obtain, differentiating implicitly $v^{2}=[p][\tau],-2 v \frac{d v}{d p}=[\tau]+[p] \frac{1}{d p_{H} / d \tau}=$ $[\tau] \frac{d p_{H} / d \tau-\sigma^{2}}{d p_{H} / d \tau}=$, verifying 2.19 by 2.18.

Carrying out these computations, one may obtain various necessary or sufficient geometric conditions on the Hugoniot curve. In particular, we have the following result. 
Corollary 2.13. Monotonicity, $d v / d p<0$ at a point $U_{+}$on the 1 -Hugoniot curve through $U_{-}$is equivalent to

(Monotone)

$$
-\frac{\bar{e}_{\tau S}}{\bar{e}_{S} \bar{e}_{\tau \tau}}<\frac{\frac{\sigma^{2}}{c^{2}}+1}{[p]}, \text { or, equivalently, } \hat{p}_{e}[p]<\sigma^{2}+c^{2} .
$$

Moreover, we have the string of implications

$$
\text { Strong' } \Rightarrow \text { Monotone } \Rightarrow \text { Lop } 1 \text { Weak' } \text {. }
$$

Proof. From (2.19), we have evidently (by $v,[\tau]<0$ ) that $d v / d p<0$ is equivalent to

$$
0>\frac{d p_{H} / d \tau-\sigma^{2}}{d p_{H} / d \tau}=2+\frac{c^{2}-\sigma^{2}}{\frac{1}{2} \hat{p}_{e}[p]-c^{2}},
$$

or, rearranging, $\hat{p}_{e}[p]<\sigma^{2}+c^{2}$ as claimed, yielding (Monotone by $[p]>0$. The logical implications (2.20) then follow by the Lax condition $|\sigma|<c$.

Remark 2.14. The implication $($ Monotone $) \Rightarrow\left(\right.$ Lop $\left._{1}\right)$ is due to C. Gardner [G]. A slightly less sharp condition pointed out by Erpenbeck [Er1] is that monotonicity of $\tau$ with respect to $s$ along the forward Hugoniot curve, $p_{S}<\frac{2 T}{-[\tau]}$ ( see Remark 3.4), or

$$
-\frac{\bar{e}_{\tau S}}{\bar{e}_{S} \bar{e}_{\tau \tau}}<\frac{\frac{2 \sigma^{2}}{c^{2}}}{[p]},
$$

also implies (Lop 1$)$, by $|\sigma| / c<1$; likewise, (2.21) implies (Monotone) and uniqueness of Riemann solutions. For other geometric implications of the various conditions, see Theorem 4.5 [MP].

Remark 2.15. From 2.20, we obtain (Strong $\Rightarrow$ Lop 1 , and Strong $\Rightarrow$ Strong?

\section{Local conditions for (in)STABility And the ASSUmptions of Smith}

The above-obtained global conditions require knowledge of the Hugoniot curve in order to compute, which is in practice quite limiting. Adapting the ingenious observations of Smith, we now show that, under some simple structural assumptions on the Hugoniot curve, these global conditions may be replaced by local conditions more convenient for analysis. In this section, we establish Theorems 1.1 and 1.3. While the latter is proved in Subsection 3.4, Theorem 1.1 is an immediate consequence of Propositions 3.1 and 3.2 and Corollary 3.6.

3.1. Local conditions and their requirements. We identify the following properties, satisfied for most standard equations of state. Denoting as the backward 1-Hugoniot through $U_{+}, H_{1}^{\prime}\left(U_{+}\right)$, the set of all left states $U_{-}$connected to $U_{+}$by a Lax 1-shock, and assuming that $H_{1}^{\prime}\left(U_{+}\right)$is a directed curve, we distinguish

$$
\begin{gathered}
{[\tau]<0 \text { on } H_{1}^{\prime}\left(U_{+}\right),} \\
p \rightarrow 0 \text { as } U \text { progresses along } H_{1}^{\prime}\left(U_{+}\right), \\
e \rightarrow 0 \text { as } U \text { progresses along } H_{1}^{\prime}\left(U_{+}\right), \\
\tau \text { is increasing and } p \text { decreasing along } H_{1}^{\prime}\left(U_{+}\right) .
\end{gathered}
$$

Recalling now conditions (Strong), (Medium $)$, (Medium $)$, (Weak of the introduction, we have the following detailed local characterizations of stability. 
Proposition 3.1. (i) Assuming ( $\mathrm{P} 1)$, (Strong) is sufficient for either stability of shock waves connecting $U_{+}$to states along the backward 1-Hugoniot or uniqueness of Riemann solutions involving states along the backward 1-Hugoniot; (ii) assuming (P2), (Weak is necessary for stability or uniqueness on the backward 1-Hugoniot. (iii) Assuming (P1)-(P3), (Medium $\mathrm{S}$ is necessary and sufficient for stability and (Medium $)$ is necessary and sufficient for uniqueness along the backward 1-Hugoniot through $U_{+}$, with, moreover,

$$
\text { Strong } \Rightarrow \text { Medium } \mathrm{U} \text { (Wedium } \mathrm{S} \text { (Weak }
$$

(iv) Assuming also ( $(\mathrm{P} 4)$, there is at most one stability transition as $U$ progresses along the backward 1-Hugoniot through $U_{+}$, that is, if the signed Lopatinski determinant turns negative once, then it stays negative.

Proof. Assertion (i) follows from (Strong') and the fact that $p>[p]$ for $p_{-} \geq 0$, assertion (ii) from (Weak') in the limit as $p_{-} \rightarrow 0$. In assertion (iii), necessity follows from (Lop)-(Lopalt) and (Monotone $]^{6}$ together with the observation that, by (2.16), $e_{+}=-\frac{1}{2} p_{+}[\tau]$ in the limit as $e_{-}, p_{-} \rightarrow 0$, so that, for $e_{-}>0, \sigma^{2}=-[p] /[\tau]=p_{+}^{2} / e_{+}$.

Sufficiency follows, similarly, from (Lop)-(Lopalt and (Monotone), and the observation that their righthand sides are monotone increasing with respect $p_{-}$and monotone decreasing with respect to $\tau_{-} \cdot 7$ so that its minimum is achieved at $p_{-}=e_{-}=0$ and $\left.\tau_{-}=\tau_{\max }:=2 e_{+} / p_{+}+\tau_{+}\right]^{8}$ the implications (3.1) follow readily from Lax condition $|\sigma|<c$. If also ( $(\overline{\mathrm{P}} 4)$ holds, then the righthand sides of all conditions are decreasing along the backward 1-Hugoniot curve, yielding (iv).

3.2. The assumptions of Smith. We now recall the assumptions of Smith and show that they imply (P1)-( $\mathrm{P} 4)$. These include, for $\tau>0$ and $S \in \mathbb{R}$, the structural conditions:

$$
\begin{gathered}
\bar{e}(\tau, S)>0 . \quad \text { (Positive energy) } \\
p=-\bar{e}_{\tau}(\tau, S)>0 . \quad \text { (Positive pressure) } \\
T=\bar{e}_{s}(\tau, S)>0 . \quad \text { (Positive temperature) } \\
p_{\tau}=-\bar{e}_{\tau \tau}(\tau, S)<0 . \quad \text { (Hyperbolicity) } \\
p_{\tau \tau}=-\bar{e}_{\tau \tau \tau}(\tau, S)>0 . \quad \text { (Genuine nonlinearity) } \\
p_{S}=-\bar{e}_{\tau S}(\tau, S)>0 . \quad \text { (Weyl condition) }
\end{gathered}
$$

Assuming (G6), we may solve $p=-\bar{e}_{\tau}(\tau, S)$ for $S=\check{S}(\tau, p)$, to obtain $e=\check{e}(\tau, p):=\bar{e}(\tau, \check{S}(\tau, p))$.

Besides the above structural assumptions, Smith imposes the asymptotic conditions:

$$
\begin{aligned}
\lim _{s \rightarrow-\infty} \bar{e}(s, \tau) & =0, \quad \lim _{s \rightarrow+\infty} \bar{e}(s, \tau)=\infty, \\
\lim _{s \rightarrow-\infty}-\bar{e}_{\tau}(s, \tau) & =0, \quad \lim _{s \rightarrow+\infty}-\bar{e}_{\tau}(s, \tau)=\infty,
\end{aligned}
$$

We shall not require Smith's further assumptions that

$$
\lim _{\tau \rightarrow 0^{+}} \check{e}(\tau, p)=\infty \text { for } p>0,
$$

\footnotetext{
${ }^{6}$ Here, we are using the standard fact that uniqueness of Riemann solutions is equivalent to monotonicity of the 1-Hugoniot curve (hence, by symmetry, the 3-Hugoniot) in terms of pressure vs. velocity [S, Sm, MP].

7 Note that dependence on $\left(\tau_{-}, p_{-}\right)$is through factors $\sigma^{2} /[p]=-1 /[\tau], \sigma /[p]=1 / \sqrt{-[p][\tau]}$, and $1 /[p]$.

8 Here we are using that, by $(2.16), \tau_{-}$is monotone decreasing with respect to both $p_{-}$and $e_{-}$, so that its maximum is achieved at the minimum $\left(p_{-}, e_{-}\right)=(0,0)$.
} 
and

$$
\lim _{\tau \rightarrow+\infty} \bar{e}(s, \tau)=0,
$$

which appear to be used for existence and not uniqueness in [S].

We complete our treatment with the following streamlined version of observations of [W], $\mathrm{S}$.

Proposition $3.2([\mathrm{~W}, \mid \mathrm{S}])$. Assumptions $(\overline{\mathrm{G} 1})-(\mathrm{G} 6),(\overline{\mathrm{H} 1})-(\overline{\mathrm{H}} 2)$ imply $(\overline{\mathrm{P} 1})-(\overline{\mathrm{P}} 4)$ : in particular, (i) the backward 1-Hugoniot is $C^{1}$, extending till $S \rightarrow-\infty, p \rightarrow 0, e \rightarrow 0$, (ii) $\tau$ and $v$ increase and $p, S$, and $\sigma^{2}$ decrease along the backward Hugoniot.

Proof. Recall that the Lax condition is $c_{-}^{2}<\sigma^{2}<c_{+}^{2}$ (with the additional condition $\sigma<0$ distinguishing 1-shocks from 3-shocks). By standard hyperbolic theory [Sm, (G4)-(G5) imply that in the vicinity of $\left(\tau_{+}, S_{+}\right)$, the backward 1-Hugoniot set of states $\left(\tau_{-}, S_{-}\right)$satisfying $(2.16)$ and the Lax condition consists of a smooth curve on which $[\tau]<0,[S]>0$. Let us focus on the region $[\tau]<0$, therefore. Rewriting the Hugoniot relation as $e_{+}-e=(1 / 2)\left(p+p_{+}\right)[\tau]$, we see readily that, assuming G1 - G2 $, \tau_{-} \leq \tau_{\max }:=2 e_{+} / p_{+}+\tau_{+}$. whence $\tau_{+} \leq \tau_{-} \leq \tau_{\max }$.

We shall show that the backward Hugoniot curve in the vicinity of $\left(\tau_{+}, S_{+}\right)$extends as a smooth curve, monotone in $\tau$ and $S$, globally on $\tau \in\left[\tau_{+}, \tau_{\max }\right), S \in\left[S_{+},-\infty\right)$ on which $(\tau, S)$ satisfy both (2.16) and the Lax condition. Moreover, we shall show that this curve contains all of the points satisfying 2.16) (without the Lax condition) on the region $[\tau]<0$. From these two latter properties, we find, reversing the roles of $\left(\tau_{-}, S_{-}\right)$and $\left(\tau_{+}, S_{+}\right)$, that any points on $[\tau]>0$ satisfying $(2.16)$ consist of reversed Lax shocks, so are not in the backward Hugoniot set, and so we can conclude that the curve we have constructed is the entire backward Hugoniot set.

Applying (G5)-G6), we find that

$$
[p]=\left(p\left(\tau_{+}, S_{+}\right)-p\left(\tau_{+}, S_{-}\right)\right)+\left(p\left(\tau_{+}, S_{-}\right)-p\left(\tau_{-}, S_{-}\right)\right) \geq p_{\tau}\left(\tau_{-}, S_{-}\right)[\tau]
$$

whenever $[S]>0$, whence $-[p] /[\tau]>p_{\tau}\left(\tau_{-}, S_{-}\right)$whenever $[\tau]<0$, or $\sigma^{2}>c_{-}^{2}$, verifying the first Lax condition so long as $[S] \geq 0,[\tau]<0$.

(i) Differentiating 2.16, we have $H_{S_{-}}=-e_{S_{-}}+\frac{1}{2} p_{S_{-}}[\tau]<0$ by (G3) and (G6), whence, by the Implicit Function Theorem, the local backward Hugoniot curve extends as a graph $S(\tau)$ so long as $S$ remains finite. Continuing, we compute that

$$
H_{\tau_{-}}=-e_{\tau_{-}}+\frac{1}{2}\left(p_{\tau_{-}}[\tau]-\left(p_{+}+p_{-}\right)\right)=\frac{1}{2}\left(p_{\tau_{-}}[\tau]-[p]\right)=\frac{1}{2}[\tau]\left(\sigma^{2}-c_{-}^{2}\right)<0
$$

so long as $[S] \geq 0$, by the (already-verified) first Lax condition, $c_{-}^{2}<\sigma^{2}$, and thus $\frac{d S_{-}}{d \tau_{-}}=-\frac{H_{\tau_{-}}}{H_{S_{-}}}<0$ so long as $[S] \geq 0$, from which we find that $\operatorname{sgn}[S]=-\operatorname{sgn}[\tau]>0$ persists along the entire curve, i.e., so long as $S$ remains finite. Rewriting the Hugoniot relation as $e_{0}-e=(1 / 2)\left(p+p_{0}\right)[\tau]$, we see readily that, assuming G1 - G2

$$
\tau_{-} \leq \tau_{\max }:=2 e_{0} / p_{0}+\tau_{0} .
$$

whence $\tau_{+} \leq \tau_{-} \leq \tau_{\max }$, with $S \rightarrow-\infty$ as $\tau \rightarrow \tau_{\max }$. Thus, the backward Hugoniot consists of the points $(\tau, S(\tau))$ for $\tau \in\left[\tau_{+}, \tau_{\max }\right)$ and this curve terminates at $(\tau, S) \rightarrow\left(\tau_{\max },-\infty\right)$ with $e, p \rightarrow 0$, by (H1) $-\mathrm{H} 2)$. Moreover, the global inequality $H_{S_{-}}<0$ implies that, as claimed, there are no other solutions of $(2.16$ for $[\tau]<0$.

(ii) We have already shown that $d S / d \tau<0$, whence we obtain also $d p / d \tau=p_{S}(d S / d \tau)+p_{\tau}<$ 0 , by $p_{\tau}<0$ and $p_{s}>0$. This verifies monotonicity of $\tau, p$, and $S$. From $[v]=-\sqrt{-[p][\tau]}$ (computed from $(\overline{\mathrm{RH}})$ ), we obtain also the stated monotonicity of $v$. Next, we compute that $\frac{d}{d S_{-}} \frac{[p]}{[\tau]}=\frac{\left.[\tau]\left(d / d S_{-}\right)[p]-p\right]\left(d / d S_{-}\right)[\tau]}{[\tau]^{2}}$ has the sign of

$$
[\tau]\left(d / d S_{-}\right)[p]-[p]\left(d / d S_{-}\right)[\tau]=\left([p]-p_{\tau}[\tau]\right)\left(d \tau / d S_{-}\right)-[\tau] p_{S_{-}}=[\tau]\left(\left(c_{-}^{2}-\sigma^{2}\right)(d \tau / d S)-p_{S}\right),
$$


or, substituting $(d \tau / d S)=-H_{S_{-}} / H_{\tau_{-}},[\tau]\left(\frac{\left(c_{-}^{2}-\sigma^{2}\right)}{\left(c_{-}^{2}-\sigma^{2}\right)} \frac{\left(-e_{S}+\frac{1}{2} p_{S}[\tau]\right)}{\frac{1}{2}[\tau]}-p_{S}\right)=-2 e_{S}<0$. Thus (since $S$ is decreasing) $\sigma^{2}=-[p] /[\tau]$ is monotone decreasing along the backward Hugoniot. Noting that the initial value of $\sigma^{2}$ at $\left(\tau_{+}, S_{+}\right)$is $c_{+}^{2}$, we have that $c_{+}^{2}>\delta^{2}$, verifying the second Lax condition along the backward Hugoniot and completing the proof.

Remark 3.3 (Monotonicity of $S, \sigma$ along forward Hugoniot). Symmetric computations show that $H_{\tau_{+}}=\frac{1}{2}\left(\sigma^{2}-c_{+}^{2}\right)[\tau]>0$ for $[\tau]<0$, where we are here using the fact already shown that the Lax conditions hold whenever $[\tau]<0$, which yields that $S$ parametrizes the forward Hugoniot curve. Similarly, $\left(d / d S_{+}\right) \frac{[p]}{[\tau]}=-2 e_{S_{+}}<0$, so that $S$ and $\sigma^{2}$ are increasing along the forward Hugoniot curve, recovering the results of Weyl [W] under assumptions (G1)-(G6).

Remark 3.4 (Monotonicity of $\tau$ along forward Hugoniot). Monotonicity of $\tau$ does not always hold in the forward direction. Differentiating (2.16) with respect to + variables, we have

$$
H_{\tau_{+}}=e_{\tau_{+}}+\frac{1}{2}\left(p_{\tau_{+}}[\tau]+\left(p_{+}+p_{-}\right)\right)=\frac{1}{2}\left(p_{\tau_{+}}[\tau]-[p]\right)=\frac{1}{2}[\tau]\left(c_{+}^{2}-\sigma^{2}\right)<0
$$

by the second Lax condition, $\sigma^{2}<c_{+}^{2}$, and $H_{S_{+}}=e_{S_{+}}+\frac{1}{2} p_{S_{+}}[\tau]$, hence $\tau$ is a monotone decreasing function of $S$ along the forward Hugoniot curve precisely so long as $-H_{S_{+}} / H_{\tau_{+}}<0$, or $p_{S}<$ $-2 T /[\tau]$. Recall that this is Erpenbeck's condition, mentioned in Remark 2.14, which is already sufficient to imply (Lop 1 ) and stability. Likewise, $v$ and $p$ need not be monotonically related.

3.3. General pressure laws. We now consider cases of general pressure laws defined in the absence of a complete equation of state $\bar{e}$. We look at two types of such laws.

3.3.1. We begin with the question of when a general pressure law

$$
p=\hat{p}(\tau, e)
$$

determines a full equation of state $e=\bar{e}(\tau, S)$, or integral of the fundamental thermodynamic relation

$$
d e=T d S-p d \tau
$$

with positive temperature $T=\bar{e}_{S}$, or, equivalently, when we may construct an entropy function $S=\hat{S}(\tau, e)$, with $S_{e}=\bar{e}_{s}^{-1}>0, S(\tau, \cdot): \mathbb{R}^{+} \rightarrow \mathbb{R}$, satisfying the transport equation

$$
S_{\tau}-\hat{p}(\tau, e) S_{e}=0 .
$$

As noted in Remark 2.7, for $\hat{p} \in C^{1}$, (3.3) may always be solved locally to particular values $\left(\tau_{0}, e_{0}\right)$, by prescribing an arbitrary monotone increasing profile $\hat{S}\left(\tau_{0}, \cdot\right)$ at $\tau_{0}$, taking $\mathbb{R}^{+} \rightarrow \mathbb{R}$, and evolving in forward and backward $\tau$ by the method of characteristics, so long as characteristics continue to cover the full half-line $e \in \overline{\mathbb{R}}^{+}$(they do not intersect, by uniqueness of solutions of the characteristic equation $d e / d \tau=-p(\tau, e))$, monotonicity being preserved by construction. Equivalently, we may prescribe an arbitrary positive temperature profile $\hat{T}\left(\tau_{0}, \cdot\right)$ at $\tau_{0}$, such that $\int(1 / T)$ de diverges at both $e \rightarrow 0^{+}$and $e \rightarrow+\infty$.

The following result, the key observation of this section, states that under the Weyl condition we may obtain also global existence in the direction of decreasing $\tau$.

Lemma 3.5. For $\hat{p} \in C^{1}\left(\mathbb{R}^{+} \times \mathbb{R}^{-}\right)$satisfying the Weyl condition $\hat{p}_{e}>0$ and $\hat{p}(\tau, \cdot) \equiv 0$, and any temperature profile $\hat{T}\left(\tau_{0}, \cdot\right)>0$ such that $\int(1 / T)$ de diverges at both $e \rightarrow 0^{+}$and $e \rightarrow+\infty$, there exists a unique entropy function $S=\hat{S}(\tau, e)$ satisfying $(3.3)$ on $0<\tau \leq \tau_{0}$, e $\in \mathbb{R}^{+}$, with $\hat{T}(\tau, e):=\left(\hat{S}_{e}\right)^{-1}>0$. Equivaelently, there is a unique equation of state $\hat{e}(\tau, S)$ defined for $0<\tau \leq \tau_{0}$ and $S \in \mathbb{R}$ with $\hat{T}=\left(\hat{S}_{e}\right)^{-1}=\hat{e}_{s}$ and $\hat{p}=-\bar{e}_{\tau}$. 
Proof. Under $\hat{p}_{e}>0$, we find that characteristics $e(\tau), d e / d \tau=-\hat{p}(\tau, e)$ diverge in the backward (decreasing $\tau$ ) direction, hence continue to cover a half-line $[a(\tau),+\infty)$. Moreover, the property $\hat{p}(\tau, \cdot) \equiv 0$ ensures that the characteristic emanating from $\left(\tau_{0}, 0\right)$ remains identically zero, so that, by continuous dependence of characteristics, $a(\tau) \equiv 0$ and the property $\lim _{e \rightarrow 0^{+}} \hat{S}(\tau, e)=-\infty$ is preserved. Meanwhile, the property $\lim _{e \rightarrow+\infty} \hat{S}(\tau, e)=+\infty$ is preserved by monotonicity of characteristics with respect to $e$ and by the fact that $\hat{S}$ by construction is constant on characteristics.

Corollary 3.6. For a $C^{2}$ pressure law $\hat{p}$, assumptions $(\mathrm{J} 1)-(\mathrm{J} 4)$ imply $(\mathrm{P} 1)-(\mathrm{P} 4)$.

Proof. Applying Lemma 3.5 for any fixed right state $\tau_{+}$, we obtain an equation of state $\bar{e}$ satisfying (G1) - G6) an (H1)-(H2) for $0<\tau \leq \tau_{\max }$, where $\tau_{\max }$ is defined as in (3.2), which generates the pressure law $\hat{p}$ through the relation $p:=-\bar{e}_{\tau}$. Noting that only $t_{+} \leq \tau \leq \tau_{\max }$ is relevant for the backward Hugoniot curve through $U_{+}$, we find by Proposition 3.2 that this implies $(\mathrm{P} 1)-(\mathrm{P} 4)$.

Remark 3.7. Under the slightly extended set of assumptions (G1)-(G6), (H1)-(H4), Smith [S] obtains not only uniqueness but also existence. It would be interesting to determine what conditions beyond (J1)-(J4) would be necessary to obtain existence for a general pressure law $\hat{p}$.

3.3.2. Secondly, we briefly consider the situation of a pressure relation

$$
p=\tilde{p}(\tau, T)
$$

defined in terms of temperature $T$ instead of internal energy $e$, for example an ideal gas law

$$
p=\tilde{p}(\tau, T):=R T / \tau, R>0 \text { constant. }
$$

Using a result of $[\mathrm{CF}]$ on the form of possible extensions of the pressure law to a complete equation of state, (see (6.77)-(6.80) of [CF]), Smith [S] shows that an ideal gas necessarily satisfies (Strong), hence the form of the pressure law imposes both uniqueness and shock stability, independent of the particular extension to a full equation of state $e=\bar{e}(\tau, S)$.

We note that similar computations may be carried out for an arbitrary relation $p=\tilde{p}(\tau, T)$; namely, conversion to a general pressure law $p=\hat{p}(\tau, e)$ amounts to the construction of an energy function

$$
e=\tilde{e}(\tau, T), \quad \tilde{e}_{T}>0
$$

from which we obtain $T=\hat{T}(\tau, e)$ by inversion with respect to $e$, and thus $\hat{p}(\tau, e):=\tilde{p}(\tau, \hat{T}(\tau, e))$. This yields the relations

$$
\hat{p}_{\tau}=\tilde{p}_{\tau}+\tilde{p}_{T} \hat{T}_{\tau}, \quad \hat{p}_{e}=\tilde{p}_{T} \hat{T}_{e}, \quad \hat{T}_{\tau}=-\frac{\tilde{e}_{\tau}}{\tilde{e}_{T}}, \quad \hat{T}_{e}=\frac{1}{\tilde{e}_{T}} .
$$

Recalling the thermodynamic relation $\hat{S}_{\tau}-p \hat{S}_{e}=0$ and $\hat{S}_{e}=\hat{T}^{-1}$, we obtain $\hat{T}_{\tau}-p \hat{T}_{e}+T \hat{p}_{e}=0$, hence, substituting from (3.4),

$$
\tilde{e}_{\tau}=T \tilde{p}_{T}-p
$$

hence $\tilde{e}_{T \tau}=\tilde{e}_{\tau T}=T \tilde{p}_{T T}$, and we can solve globally for a positive temperature $e$ and derivative $e_{T}$ for $\tau \geq \tau_{0}$ provided $\tilde{p}_{T T} \geq 0$, and $\tilde{p}(\tau, 0) \equiv 0$, which together imply also $T \tilde{p}_{T}-p \geq 0$. For further discussion, particularly of relation (3.5), see the discussion of Helmholtz energy in Appendix B.

In the ideal gas case, we have equality, $\tilde{p}_{T T}=T \tilde{p}_{T}-p=0$, so find that $\tilde{e}(\tau, T)=g(T)$ depends on $T$ alone, where $g$ is any monotone increasing function one-to-one on $\mathbb{R}^{+}$to $\mathbb{R}^{+}$. We thus have $\hat{T}_{\tau}=0$, and, from (3.4), $\hat{p}_{\tau}=\tilde{p}_{\tau}$, so that Strong is equivalent to $\tilde{p}_{\tau}<0$, independent of the choice of $g$. Moreover, we have by direct computation $\tilde{p}_{\tau}=-R T / \tau^{2}<0$, recovering somewhat more simply the result of Smith.

However, note that any admissible temperature function $\tilde{e}(\tau, T)$ may be modified by the addition of any nondecreasing function $h(T)$ with $h(0)=0$ to yield another admissible temperature function 
$\tilde{e}_{1}(\tau, T):=\tilde{e}(\tau, T)+h(T)$. Moreover, except in the special situation $\tilde{e}_{\tau} \equiv 0$ just considered, or when $\tilde{p}_{T} \equiv 0$, such modification will result in a change in $\hat{T}_{\tau}$ and $\hat{p}_{\tau}$, so that satisfaction of our conditions (Weak), Strong, (Medium $)$, and (Medium $\mathrm{U}$ may be affected by the choice of $\tilde{e}$, in contrast with the situation of pressure laws of "primary" type $p=\hat{p}(\tau, e)$.

Lemma 3.8. In terms of a pressure law $\tilde{p}(\tau, T)$, hyperbolicity becomes $\tilde{p}_{\tau}<\frac{T \tilde{p}_{T}^{2}}{\tilde{e}_{T}}$, or

$$
\hat{p}_{\tau}=\tilde{p}_{\tau}-\tilde{p}_{T} \tilde{e}_{\tau} / \tilde{e}_{T}<p \tilde{p}_{T} / \tilde{e}_{T}=p \hat{p}_{e},
$$

while Strong and Weak become, respectively

$$
\hat{p}_{\tau}=\tilde{p}_{\tau}-\tilde{p}_{T} \tilde{e}_{\tau} / \tilde{e}_{T}<0 \text { (strong) and } \hat{p}_{\tau}=\tilde{p}_{\tau}-\tilde{p}_{T} \tilde{e}_{\tau} / \tilde{e}_{T}<p \tilde{p}_{T} / 2 \tilde{e}_{T}=p \hat{p}_{e} / 2 \text { (weak). }
$$

Proof. Immediate, from (3.4),

Remark 3.9. These findings are useful for the general van der Waals gas law $\left(p+a / \tau^{2}\right)(\tau-b)=R T$, $a, b, R>0$, where $\tilde{p}(\tau, T)=\frac{R T}{\tau-b}-\frac{a}{\tau^{2}}$. This will be pursued elsewhere.

3.4. Link to the Riemann Problem. The previous subsections have revealed a certain parallel between the transition from stability to instability for individual shocks on the hand and that from uniqueness to non-uniqueness for the Riemann problem on the other. Clearly, these two transitions are not generally equivalent to each other: there are equations of state for which all shocks have positive signed Lopatinski determinant and yet for certain data, the Riemann problem has more than one solution. On the other hand, according to Theorem 1.1, the mere existence of shocks with negative signed Lopatinski determinant readily implies the existence of Riemann data admitting several solutions. This section serves to make this connection transparent.

As pointed out already by C. Gardner [G] in the context of gas dynamics, vanishing of the Lopatinski determinant has a natural interpretation in terms of bifurcation of Riemann solutions.

Proof of Theorem 1.3. For single-shock data, the Lopatinski determinant coincides with the determinant of the local Jacobian of Lax's wave map [L]. The assertion now follows from the following general fact that we briefly prove as we know no reference for it.

Lemma 3.10. For any $C^{1}$ mapping $F: \mathbb{R}^{n} \rightarrow \mathbb{R}^{n}$, local uniqueness prevents $\operatorname{det}(D F)$ from undergoing a local strict change of sign.

Proof. Local uniqueness at a point $p$ implies the existence of an open ball $B$ around $p$ such that $F(B) \cap F(\partial B)=\emptyset$. With $d$ denoting the Brouwer degree, the function $B \rightarrow \mathbb{R}, q \mapsto d(F, B, F(q))$ is thus a constant. As $d(F, B, F(q))=\operatorname{sgn} \operatorname{det} D F(q)$ whenever $\operatorname{det} D F(q) \neq 0, B$ cannot simultaneously contain points $q^{ \pm}$with $\pm \operatorname{det} D F\left(q^{ \pm}\right)>0$.

Remark 3.11 (Stability as selection principle). The above-described bifurcation analysis bears on the question posed in [MP] whether shock stability could serve in the situation of multiple Riemann solutions as a selection principle. For, generically, the bifurcation local to the single-shock solution should be of fold type, with $\Delta$ taking different signs (corresponding to opposite orientations) on either side of the fold; see section 6.2, [Z1], for an analysis of the general $2 \times 2$ case, and [B-G] for an extension to the $3 \times 3$ gas dynamical case. Assuming that additional small-amplitude waves in other family are stable, as is expected to be the case, we find that sign of $\Delta$, and thus stability of the large component shock near $\left(U_{-}, U_{+}, \sigma\right)$, determines stability of the associated Riemann patterns, so that, at the level of Riemann patterns, this is a transcritical bifurcation featuring exchange of stability from one branch to another, and indeed serves as a selection principle. 


\section{Gas-dynamical examples and COUnterexamples}

4.1. Global counterexample. Recall the equation of state

$$
\bar{e}(\tau, S)=\frac{e^{S}}{\tau}+C^{2} e^{S / C^{2}-\tau / C}, \quad C>>1
$$

asserted to exhibit instability in Theorem A.

4.1.1. Proof of Theorem 1.2. The function $\bar{e}$ is evidently convex. Computing, we have

$$
\begin{gathered}
T=\bar{e}_{S}=e^{S} / \tau+e^{S / C^{2}-\tau / C}>0, \\
p=-\bar{e}_{\tau}=e^{S} / \tau^{2}+C e^{S / C^{2}-\tau / C}>0, \\
p_{S}=-\bar{e}_{S \tau}=e^{S} / \tau^{2}+C^{-1} e^{S / C^{2}-\tau / C}<0, \\
p_{\tau}=-\bar{e}_{\tau \tau}=-2 e^{S} / \tau^{3}-e^{S / C^{2}-\tau / C}<0, \\
p_{\tau \tau}=-\bar{e}_{\tau \tau \tau}=6 e^{S} / \tau^{4}+C^{-1} e^{S / C^{2}-\tau / C}>0,
\end{gathered}
$$

whence this model satisfies the basic properties (G1)-G6 assumed by Smith: positivity of $e, T$, $p$, genuine nonlinearity, and the Weyl condition.

Continuing, it is readily seen that

$$
\lim _{S \rightarrow-\infty} \bar{e}(S, \tau)=0, \quad \lim _{S \rightarrow+\infty} \bar{e}(S, \tau)=\infty, \text { and } \lim _{\tau \rightarrow 0^{+}} \bar{e}(S, \tau)=\infty, \quad \lim _{\tau \rightarrow+\infty} \bar{e}(S, \tau)=0,
$$

validating Smith's asymptotic hypotheses (H1) and (H2). Boundedness of $p$ as $\tau \rightarrow 0^{+}$clearly implies that $S \rightarrow-\infty$, or else the first term $e^{S} / \tau^{2}$ would blow up. Noting that for $e^{S} / \tau^{2} \leq C, e^{S} / \tau \leq$ $C \tau \rightarrow 0$ as $\tau \rightarrow 0^{+}$, we thus obtain $e \rightarrow 0$, verifying Smith's hypothesis (H3): $\lim _{\tau \rightarrow 0^{+}} \hat{e}(p, \tau)=0$. Likewise, (H4) is readily verified: $\lim _{\tau \rightarrow+\infty} \bar{e}(s, \tau)=0$. Thus, $\bar{e}$ satisfies all of Smith's hypotheses (G1)- G6 and (H1)-(

By Proposition 3.2 and Remark 3.4, we thus obtain monotonicity of $S$ and $\sigma$ along forward and backward Hugoniot curves. Evaluating at $\tau_{+}=1, S_{+}=0$, and using the assumed asymptotics $C>1$, we obtain

$$
\frac{-\bar{e}_{S \tau}}{\bar{e}_{\tau \tau}}-\frac{2 \bar{e}_{S}}{-e_{\tau}}=\frac{1+O\left(C^{-1}\right)}{3+O\left(C^{-1}\right)}-\frac{2+O\left(C^{-1}\right)}{C+O(1)} \sim \frac{1}{3}>0,
$$

giving failure of Smith's condition (Weak).

4.1.2. Numerical illustration In Figures 1 and 2 below, we illustrate the theory presented in Theorem A by numerical computation, displaying the forward and backward Hugoniot curves for an unstable 1-shock of (1.3), with endstates $\left(\tau_{+}, S_{+}\right)=(1,0)$ and $\left(\tau_{-}, S_{-}\right)=(12.089548257354499,-6)$, described as plots of $\tau, p, v, e, \sigma$ against $S$. Here, the Hugoniot curve is obtained numerically by solving (2.16) for $\tau$ as a function of $S$ using the bisection method. The transition to instability is computed along the backward Hugoniot using (Medium $\mathrm{S}$.

4.2. Local counterexample. Taylor expanding the equation of state 4.1 in the quantitiy $1 / C$ about the limiting value $1 / C=0$ as $C \rightarrow \infty$, we obtain the simplified, local model

$$
\bar{e}(\tau, S)=e^{S} / \tau+S-C \tau+\tau^{2} / 2, \quad C>>1,
$$

described in Remark 1.5. It is readily seen that the flow of $(2.8)$ is independent of the value of $C$, with $C$ only serving to shift pressure by positive $C$ but not affecting dynamics. Thus, dropping the assumptions of positive pressure and energy, we may replace 4.2 by the canonical model

$$
\bar{e}(\tau, S)=e^{S} / \tau+S+\tau^{2} / 2
$$


(a)

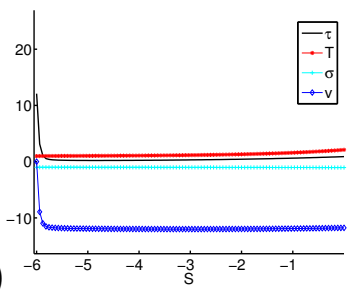

(d)

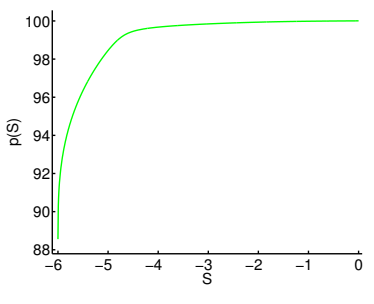

(b)

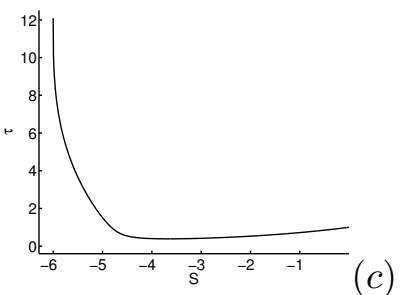

$(e)$

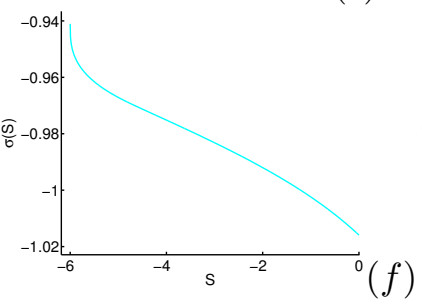

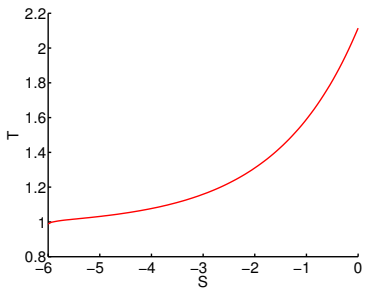

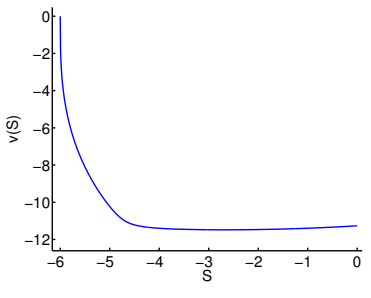

Figure 1. (a) The forward 1-Hugoniot curve through $\left(\tau_{-}, S_{-}\right) \approx(12.09,-6)$ for global model $\bar{e}(\tau, S)=e^{S} / \tau+e^{S / C^{2}-\tau / C}$ of points $(\tau, S)$ to which $\left(\tau_{-}, S_{-}\right)$connects by a Lax 1-shock, displayed as a graph $(\tau, p, v, e, \sigma)$ over $S$ plotted with respective colors (black, green, blue, red, cyan). We zoom in to see (b) the Hugoniot curve, (c) $T$ over $S$, (d) $p$ over $S$, (e) $\sigma$ over $S$, and (f) $v$ over $S$. Note nonmonotonicity of $\tau$ and $v$ with respect to $S$.

(a)

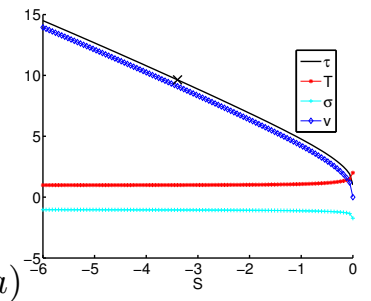

(d)

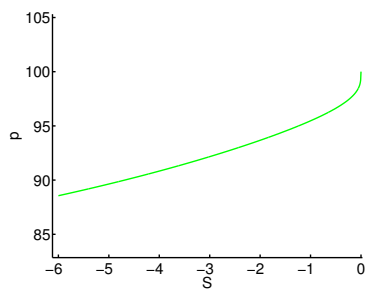

(b)

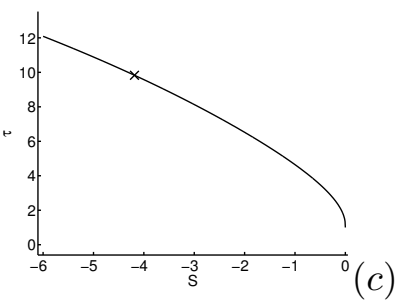

$(e)$

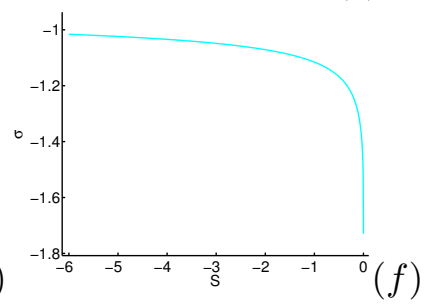

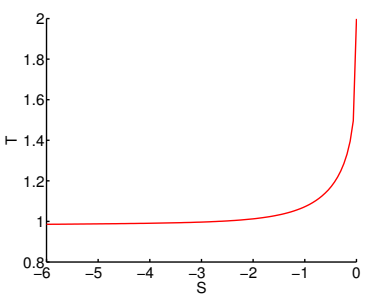

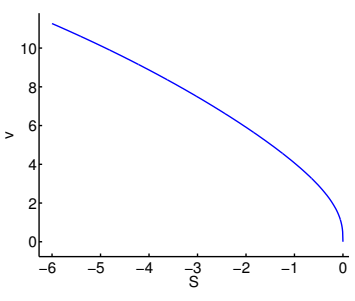

Figure 2. The backward 1-Hugoniot curve through $\left(\tau_{+}, S_{+}\right)=(1,0)$ for global model $\bar{e}(\tau, S)=e^{S} / \tau+e^{S / C^{2}-\tau / C}$ of points $(\tau, S)$ connecting to $\left(\tau_{+}, S_{+}\right)$by a Lax 1shock, displayed as a graph $(\tau, p, v, e, \sigma)$ over $S$ plotted with respective colors (black, green, blue, red, cyan). We zoom in to see (b) the Hugoniot curve, (c) $T$ over $S$, (d) $p$ over $S$, (e) $\sigma$ over $S$, and (f) $v$ over $S$. The value of $\left(\tau_{-}, S_{-}\right)$along the backward Hugoniot curve at which transition to instability occurs is marked by a black X.

for which $C$ no longer appears, capturing the large- $C$ behavior of $(1.3)$. This is perhaps the simplest model exhibiting a convex entropy, monotonicity of shock speed and $S$ along the forward Hugoniot, but also unstable Lax shock waves.

Model (4.3) satisfies (G1) and (G3)-(G6), but not $(\bar{G} 2),(\sqrt{\mathrm{H} 1})-(\sqrt{\mathrm{H}} 2)$, or $(\overline{\mathrm{P} 1})-(\overline{\mathrm{P}} 3)$. From $(\mathrm{G} 3)-$ (G6), we find by the proof of Proposition 3.2 that the backward Hugoniot through any $\left(\tau_{+}, S_{+}\right)$ extends for all $S \in\left[S_{+},-\infty\right)$ as a graph of $\tau$ as a function of $S$, on which the Lax conditions hold 

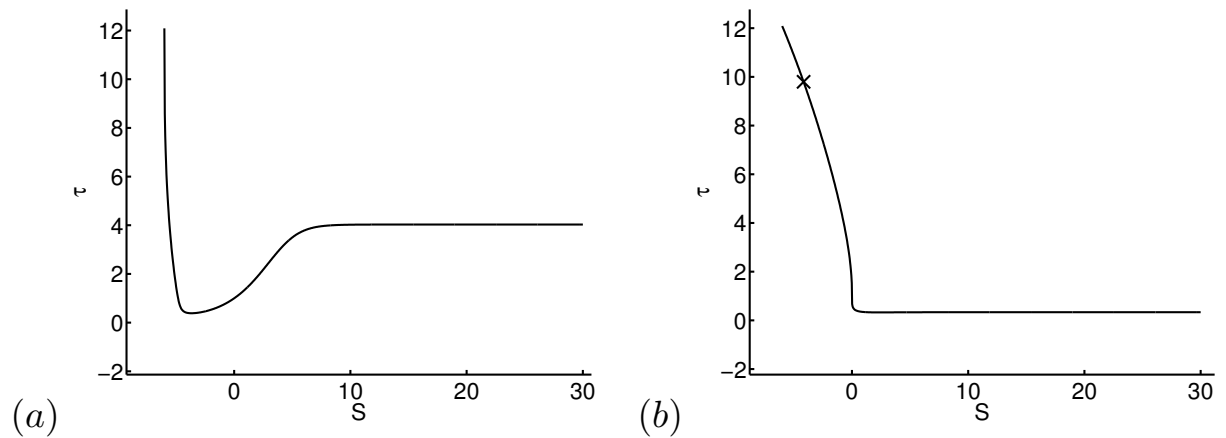

Figure 3. Extended plots of the (a) forward and (b) backward Hugoniot curves shown in Figures 1 and 2 for global model $\bar{e}(\tau, S)=e^{S} / \tau+e^{S / C^{2}-\tau / C}$.

and $p, \sigma^{2}$ are monotone decreasing; likewise, the forward Hugoniot is parametrized by increasing $S$, with $\sigma$ monotone decreasing ( $\sigma^{2}$ increasing). However, $p$ and $e$ do not approach zero as $S \rightarrow-\infty$, instead becoming negative.

Indeed, the Hugoniot curve for (4.2) - 4.3 may be solved explicitly as a cubic in $\tau$. For example, setting $\left(\tau_{+}, S_{+}\right)=(1,0)$ and noting that $\bar{p}(\tau, S)=-\bar{e}_{\tau}=\frac{\mathrm{e}^{S}}{\tau^{2}}-\tau$, we find that $p_{+}=0$ and $e_{+}=3 / 2$. Substituting into $H(\tau, s)=e-e_{+}+(1 / 2)\left(p+p_{+}\right)\left(\tau-\tau_{+}\right)=0$ yields

$$
H(\tau, S)=\frac{\mathrm{e}^{S}}{\tau}+S+1 / 2 \tau^{2}-3 / 2+1 / 2\left(\frac{\mathrm{e}^{S}}{\tau^{2}}-\tau\right)(\tau-1)=0
$$

or, multiplying by $\tau^{2}$,

$$
\tau^{3}+3 \mathrm{e}^{S} \tau+(2 S-3) \tau^{2}-\mathrm{e}^{S}=0 .
$$

Thus an explicit solution $\tau(S)$ is available through the cubic formula. Moreover, along the backward Hugoniot, we find as $S \rightarrow-\infty$ that 4.4 reduces asymptotically to $\tau^{3}+2 S \tau^{2}=0$, so that, asymptotically, $\tau(S) \sim-2 S \rightarrow+\infty$, and $e \sim 2 S^{2} \rightarrow+\infty, p \sim-\tau \rightarrow-\infty$.

Proposition 4.1. For model (4.3) (or, equivalently, (4.2), the backward Hugoniot through any $\left(\tau_{+}, S_{+}\right)$extends for all $S \in\left[S_{+},-\infty\right)$ as a graph of $\tau$ as a function of $S$, on which the Lax conditions hold, $\tau$ is monotone increasing, and $p, \sigma^{2}$ are monotone decreasing; likewise, $\sigma$ is monotone decreasing and $S$ monotone increasing along the forward Hugoniot. Yet, for $\left(\tau_{+}, S_{+}\right)=(1,0)$, there exist $\left(\tau_{-}, S_{-}\right)$along the backward Hugoniot such that the Lax 1-shock connecting $(\tau, S)_{ \pm}$is Lopatinski (i.e., Hadamard) unstable.

Proof. By direct computation (see further calculations just below), we obtain

$$
\left(\frac{-\bar{e}_{S \tau}}{\bar{e}_{\tau \tau}}-\frac{2 \bar{e}_{S}}{[p]}\right)_{(\tau, S)=\left(\tau_{+}, S_{+}\right)}=\frac{1}{3}-\frac{4}{-p_{-}} \sim \frac{1}{3}>0
$$

as $S \rightarrow-\infty, \tau \rightarrow+\infty$, and $p_{-} \rightarrow-\infty$, yielding the result by Proposition 2.10 and failure of (Weak'.

4.2.1. Estimating the stability transition. From (4.3), we obtain

$$
p=e^{S} / \tau^{2}-\tau, \quad e_{S}=e^{S} / \tau+1, \quad e_{\tau \tau}=2 e^{S} / \tau^{3}+1, \quad e_{\tau S}=-e^{S} / \tau^{2},
$$

hence, evaluating at $\left(S_{+}, \tau_{+}\right)=(0,1)$,

$$
p_{+}=0, \quad\left(e_{S}\right)_{+}=2, \quad\left(e_{\tau \tau}\right)_{+}=3, \quad\left(e_{\tau S}\right)_{+}=-1,
$$


so that $\phi:=-\left.\frac{\bar{e}_{S} \bar{e}_{\tau \tau}}{\bar{e}_{\tau S}}\right|_{\left(S_{+}, \tau_{+}\right)}=-\frac{(2)(3)}{-1}=6$, and $\theta:=1+\sqrt{\frac{[p] /[\tau]}{p_{\tau}}}=1+\sqrt{\frac{p}{-3\left(\tau_{-}-1\right)}}$.

Thus, change in stability correponds to change in sign of

$$
\delta:=p_{+}-p_{-}-\theta \phi=-\frac{\mathrm{e}^{S}}{\tau^{2}}+\tau-6-2 \sqrt{-\left(3 \frac{\mathrm{e}^{S}}{\tau^{2}}-3 \tau\right)(\tau-1)^{-1}} .
$$

Noting that $p \approx-\tau$, for $S_{-}$large and negative, so that $[p] /[\tau] \approx \frac{-\tau_{-}}{\tau_{-}-\tau_{+}}$, and recalling that $c_{+}^{2}=$ $-p_{\tau,+}=3$ we may well approximate $\theta \approx 1+\sqrt{\left.\frac{\tau_{-}}{6\left(\tau_{-}-1\right.}\right)} \approx 1.6$, so that the stability transition occurs approximately at $\tau_{-}=9.6$ and, estimating $S \approx \frac{1}{2}(3-\tau)$ for $e^{S}<<1$ in 4.4 , $S_{-} \approx-3.3$. In fact, numerically we determine that $\delta=0$ occurs for $S_{-} \in\left(S_{1}, S_{2}\right)=(-3.3348293,-3.334829)$, $\tau_{-} \in\left(\tau_{2}, \tau_{1}\right)=(9.6589763,9.6589769)$. See Figure 4 below for a plot of the Hugoniot curve computed by the Bisection Method, with the stability transition indicated on the curve.

Remark 4.2. From $c_{+}^{2}=3, \sigma^{2}=-\frac{[p]}{[\tau]} \sim \frac{\tau_{-}}{\tau_{-}-\tau_{+}} \sim 1+\frac{1}{\tau_{-}}$, and $c_{-}^{2}=1+O\left(e^{S_{-}}\right)$, we verify directly the Lax conditions $c_{-}^{2}<\sigma^{2}<c_{+}^{2}$ for $S_{-}>>1$, with the shock nearly characteristic at $U_{-}$.

(a)

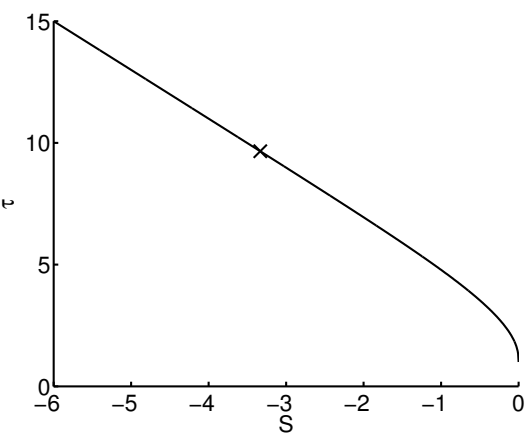

(b)

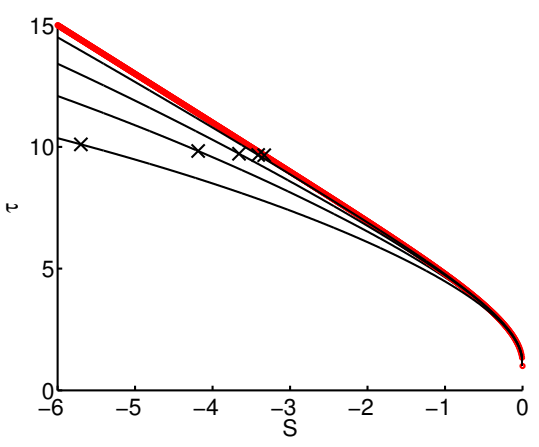

Figure 4. We mark transition to instability with a thick X. (a) Plot of the Hugoniot curve for the local model, $\bar{e}(S, \tau)=e^{S} / \tau+S+\tau^{2} / 2,\left(\tau_{0}, S_{0}\right)=(1,0)$. (b) Plot of the the Hugoniot curve for the local model with a thick red line and that of the global model for $C=40,100,250$. Note that as $C \rightarrow \infty$, the Hugoniot curve of the local model matches well that of the global model.

4.3. Stable example. For comparison, consider finally the closely related model

$$
\bar{e}(\tau, S)=e^{S} / \tau-C \tau+\tau^{2} / 2, \quad C>>1 .
$$

Here, likewise the flow of 2.8 is independent of the value of $C$, so that it is equivalent to consider the simpler version

$$
\bar{e}(\tau, s)=e^{S} / \tau+\tau^{2} / 2 .
$$

Proposition 4.3. For model 4.6) (or, equivalently, 4.5), the Hugoniot through any $\left(\tau_{+}, S_{+}\right)$ extends for all $S \in(-\infty,-\infty)$ as a monotone graph of $\tau$ as a function of $S$, on which the Lax conditions hold precisely for $[\tau]<0$. Moreover, all Lax shocks have positive signed Lopatinski determinant.

Proof. Again, from (G3)-(G6), we find by the proof of Proposition 3.2 that the backward Hugoniot through any $\left(\tau_{+}, S_{+}\right)$extends for all $S \in\left[S_{+},-\infty\right)$ as a graph of $\tau$ as a function of $S$, on which the Lax conditions hold and $p, \sigma^{2}$ are monotone decreasing; by similar reasoning, the forward Hugoniot 
is parametrized by increasing $S$, with $\sigma$ monotone decreasing ( $\sigma^{2}$ increasing). Indeed, the Hugoniot curve for 4.6) can be determined explicitly by solving

$$
\begin{aligned}
0=H(\tau, S) & =e^{S} / \tau+\tau^{2} / 2-e_{+}-\frac{1}{2}\left(e^{S} / \tau^{2}-\tau+p_{+}\right)\left(\tau_{+}-\tau\right) \\
& =e^{S} / \tau-e_{+}-\frac{1}{2}\left(e^{S} / \tau^{2}+p_{+}\right)\left(\tau_{+}-\tau\right)+\frac{1}{2} \tau \tau_{+}
\end{aligned}
$$

or, using the identities $p_{+}+\tau_{+}=\frac{e^{S_{+}}}{\tau_{+}}$and $e_{+}+\frac{p_{+} \tau_{+}}{2}=\frac{3}{2} \frac{e^{S_{+}}}{\tau_{+}}, e^{S-S_{+}}=\left(\frac{3 \tau_{+}-\tau}{3 \tau-\tau_{+}}\right) \frac{\tau^{2}}{\tau_{+}^{2}}$, for

$$
S-S_{+}=\log \left(\frac{3 \tau_{+}-\tau}{3 \tau-\tau_{+}}\right) \frac{\tau^{2}}{\tau_{+}^{2}}
$$

Computing $(d / d \tau)\left(S-S_{+}\right)=-\frac{6(x-1)^{2}}{x(3-x)(3 x-1) \tau_{+}}<0$ for $x:=\tau / \tau_{+}$and $1 / 3<x<3$, we find that $S$ is a monotone function of $\tau$ along the entire (forward and backward) Hugoniot curve. But, monotonicity along the forward Hugoniot implies stability by Remarks 2.14 and 3.4 .

$(a)$

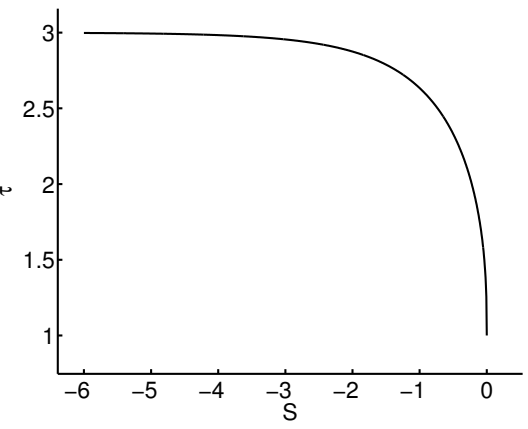

(b)

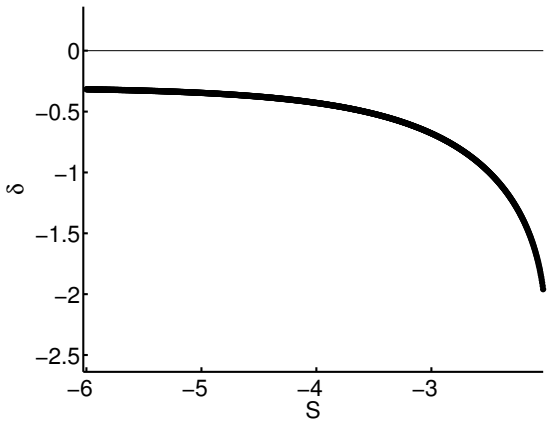

Figure 5. (a) Plot of the Hugoniot curve for the local model $\bar{e}(S, \tau)=e^{S} / \tau-$ $C \tau+\tau^{2} / 2$ with $C=100,\left(\tau_{0}, S_{0}\right)=(1,0)$. (b) Plot of $\delta$ against $S$ where $\delta<0$ corresponds to stability.

\section{PART II. VISCOUS STABILITY}

Stepping outside the inviscid setting, we can extract further interesting information from the sign of the signed Lopatinski determinant. This fact and the Evans function, to which it refers, are discussed in Section 5. Section 6 presents the findings that were summarized as Numerical Observation A in Sec. 1. The phenomenologically most interesting piece of Part II might be Section 7, where the findings of Numerical Observation B are detailed, on the rich stability behavior of shock waves in an artifically designed system.

\section{The Evans Function}

We now turn to the study of viscous stability, or stability of continuous traveling shock fronts

$$
w(x, t)=\bar{w}(x-\sigma t), \quad \lim _{z \rightarrow \pm \infty} \bar{w}(z)=U_{ \pm}
$$

of a hyperbolic-parabolic system of conservation laws

$$
f^{0}(w)_{t}+f(w)_{x}=\left(B(w) w_{x}\right)_{x},
$$


corresponding to an inviscid shock $\left(U_{-}, U_{+}, \sigma\right)$ of (2.1), that is, a solution of the traveling-wave ODE

$$
B(\bar{w}) \bar{w}^{\prime}=f(\bar{w})-\sigma f^{0}(\bar{w})-\left(f\left(U_{-}\right)-\sigma f^{0}\left(U_{-}\right)\right) .
$$

Recall [GZ, ZZ, MaZ1, MaZ2, Z1, BLeZ, Z5] that, under rather general circumstances, shared by most equations of continuum mechanics- in particular, the equations of gas dynamics, MHD, and viscoelasticity- Lax-type traveling-wave profiles $\bar{w}$, when they exist, (i) are generically smooth and converging at exponential rate as $z \rightarrow \pm \infty$ to their endstates $U_{ \pm}$, and (ii) are nonlinearly orbitally stable as solutions of (5.2) if an only if they satisfy an Evans function condition:

$$
D(\lambda) \text { has no zeros on } \Re \lambda \geq 0 \text { except for a multiplicity one zero at } \lambda=0,
$$

where the Evans function $D$ is a Wronskian at $x=0$ of appropriately chosen bases of solutions of the linearized eigenvalue equations about $\bar{w}$ that decay at plus and minus spatial infinity, respectively, that is analytic in $\lambda$ on a neighborhood of $\Re \lambda \geq 0$, with complex symmetry $\overline{D(\lambda)}=D(\bar{\lambda})$, whose zeros agree on $\{\Re \lambda \geq 0\} \backslash\{0\}$ in location and multiplicity with the eigenvalues of the linearized operator about the wave.

5.1. Viscous vs. inviscid stability. Like inviscid stability, viscous stability holds always for Lax type shocks in the small-amplitude limit [ZH, HuZ3, [PZ, FS1, FS2]. Moreover, as found in GZ, ZS], we have the fundamental relation

$$
D(\lambda)=\lambda \nu \delta+O\left(\lambda^{2}\right)
$$

for $\lambda$ sufficiently small, where $\nu$ is a Wronskian associated with the linearized traveling-wave ODE, with nonvanishing of $\nu$ corresponding to transversality of the connecting viscous profile, and $\delta$ is the Lopatinski determinant (2.4) associated with the inviscid stability problem. Without loss of generality, normalize $\nu$ and $\delta$ so as to be real. Then, moving along the Hugoniot curve in the direction of increasing amplitude, we find that, so long as there persists a transversal traveling-wave connection, so that $\nu$ cannot change sign, that real zeros of the Evans function can cross through the origin into the unstable half-plane if and only if the Lopatinski determinant $\delta$ changes sign, that is, there is a corresponding stability transition at the inviscid level $[\mathrm{GZ}, \mathrm{ZH}, \mathbf{Z S}, \mathbf{Z 1}, \mathbf{Z 3}$.

More precisely, we see that, assuming persistence of transversal connections, inviscid stability transitions correspond at viscous level to the passage of an odd number of unstable roots through the origin, so can never occur without a corresponding viscous stability transition. On the other hand, there can occur viscous stability transitions corresponding to passage of an even number of real roots, or of one or more complex conjugate pairs of unstable roots through the imaginary axis, which are not detected at the inviscid level. Thus, viscous stability is a logically stronger condition than inviscid stability [ZS. Our goal in this part of the paper is to investigate whether this logical implication can be strict for systems (5.2) possessing an associated convex entropy, and in particular whether complex conjugate pairs of roots can cross, signaling Hopf bifurcation to time-periodic "galloping" behavior [TZ1, TZ2, TZ3, BeSZ].

5.2. Numerical stability analysis. The Evans function, or, for that matter, the underlying profile (5.1), is only rarely computable analytically. However, as described, e.g., in [Br, BrZ, HuZ1, Z4, these may be approximated numerically in a well-conditioned and efficient manner. Here, we carry out numerical experiments using MATLAB and the MATLAB-based Evans function package STABLAB [BHZ2] developed for this purpose by Barker, Humpherys, Zumbrun, and collaborators. More precisely, we study the integrated Evans function $\tilde{D}(\lambda)$, a Wronskian associated with the integrated eigenvalue equations, whose zeros on $\{\Re \lambda \geq 0\} \backslash\{0\}$ likewise agree in location and 
multiplicity with the eigenvalues of the linearized operator about the wave, but which does not have a zero at the origin, obeying a small-frequency expansion

$$
\tilde{D}(\lambda)=\nu \delta+O(\lambda)
$$

in place of $(5.5)$.

Descriptions of the underlying algorithms and other information useful in reproducing these computations may be found in Appendix C.

\section{Gas Dynamical EXAMPLES}

The compressible Navier-Stokes equations, augmenting (2.8) with the transport effects of viscosity and heat conduction, appear in Lagrangian coordinates as

$$
\begin{aligned}
\tau_{t}-v_{x} & =0 \\
v_{t}+p_{x} & =\left(\frac{\mu v_{x}}{\tau}\right)_{x}, \\
\left(e+v^{2} / 2\right)_{t}+(v p)_{x} & =\left(\frac{\mu v v_{x}}{\tau}\right)_{x}+\left(\frac{\kappa T_{x}}{\tau}\right)_{x},
\end{aligned}
$$

where $\tau$ denotes specific volume, $v$ velocity, $e$ specific internal energy, $p$ pressure, and $T$ temperature, and $\mu$ and $\kappa$ are coefficients of viscosity and heat conductivity, here taken constant.

To close these equations requires both a pressure law $p=\hat{p}(\tau, e)$ and a temperature law $T=$ $\hat{T}(\tau, e)$, in contrast to the case of the Euler equations $(2.8)$, that is, a complete equation of state. Indeed, the most convenient formulation for our purposes here is in terms of the variable $w:=$ $(\tau, v, T)$ replacing $e$ with $T$. We will use equations of state given in the form

$$
p=\check{p}(\tau, T), \quad e=\check{e}(\tau, T)
$$

arising through the Helmholtz energy formulation described in Appendix B, for which the equations take the convenient block structure described in Appendix C.1. Note that besides hyperbolicity, $\bar{p}_{\tau}<0$, the Navier-Stokes equations (6.1) require also parabolicity,

$$
\bar{e}_{S S}=(\partial T / \partial e)(\partial e / \partial S)>0,
$$

in order to be well-posed, which is the condition needed to obtain laws 6.2 from a given energy function $e=\bar{e}(\tau, S)$ of the form considered in previous sections.

Substituting (5.1) into (5.2) and solving the first equation of (5.3) for

$$
w_{1}=\tau=\tau_{-}+\sigma^{-1}\left(v_{-}-v\right)
$$

as described in Appendix C.1. we obtain for a profile corresponding to an inviscid shock $\left(U_{-}, U_{+}, \sigma\right)$ of (2.8) the traveling-wave equations

$$
\tau^{-1}\left(\begin{array}{cc}
\mu & 0 \\
\mu v & \kappa
\end{array}\right)\left(\begin{array}{c}
v \\
T
\end{array}\right)^{\prime}=\left(\begin{array}{c}
-\sigma\left(v-v_{-}\right)+p-p_{-} \\
-\sigma\left(e+v^{2} / 2-e_{-}-v_{-}^{2} / 2\right)+p v-p_{-} v_{-}
\end{array}\right) .
$$

Proposition 6.1 (Gi]). For a $C^{2}$ energy function $\bar{e}(\tau, S)$ satisfying (G3)-(G6), every Lax type shock of (2.8) has a unique continuous shock profile (5.1) of (6.1)-(6.2), which is a transversal connection of (6.4) converging exponentially to endstates $U_{ \pm}$as $x \rightarrow \pm \infty$ in up to two derivatives.

Proof. More generally, Gilbarg shows that, assuming the Weyl condition $\bar{p}_{S}>0$, a Lax-type inviscid 1-shock for which the pressure at the righthand state $U_{+}$is minimal among all connections to $U_{-}$ with the same shock speed $\sigma$ possesses a unique viscous profile (5.1). Since (G3)-(G6) imply uniqueness of inviscid Lax shocks for a given speed, we thus obtain existence of a unique profile; transversality then follows by the observation that this connection must be of saddle-node type, so transversal whenever it exists and exponentially decaying by standard stable/unstable manifold theory. See also [W] and [MP, Appendix C]. 
Proposition 6.2 (Kaw,KSh). For a convex $C^{2}$ energy function $\bar{e}(\tau, S)$ satisfying (G3) -(G6), (6.1) possesses the convex viscosity-compatible entropy $\hat{S}(\tau, e)$, globally on $\tau, e>0$.

Proof. See, e.g., [Kaw, KSh, MP, Z1, Z2].

6.1. Numerical details. Despite the familiarity of the gas dynamical equations (6.1), and the straightforward analytical treatment under the assumptions we impose, we find the numerical Evans function analysis of our gas dynamical examples to be quite challenging, due to the presence of multiple scales. Indeed, this turns out to be the most computationally intensive problem that we have so far considered, even including the famously intensive problem of detonation stability considered in [Er3, LS, BHLyZ1, BZ2]. We thus find it necessary to include some extra details on how the computations are carried out, despite that this is not our main emphasis here.

6.1.1. Computation of profiles. To approximate numerically the profiles guaranteed by Proposition 6.1 for a given equation of state $\bar{e}(\tau, S)$, we see two basic possible approaches. The more direct, but numerically somewhat cumbersome approach is to evaluate the functions $\check{p}(\tau, T)=\bar{p}(\tau, \check{S}(\tau, T)$ and $\check{e}(\tau, T)=\bar{e}\left(\tau, \check{S}(\tau, T)\right.$ by numerically inverting the relation $T=\bar{e}_{S}(\tau, S)$ to solve for $S=\check{S}(\tau, T)$, using $\bar{e}_{S S}>0$. As computation of the profile is a one-time cost, the associated cost in function calls is perhaps not too serious, but this still seems preferable to avoid.

The approach we follow here is, rather, to compute the profile in $(\tau, S)$ coordinates using the change of variables formula $\left(\begin{array}{c}\tau \\ S\end{array}\right)^{\prime}=\left(\begin{array}{cc}-\sigma & 0 \\ \bar{e}_{S \tau} & \bar{e}_{S S}\end{array}\right)^{-1}\left(\begin{array}{c}v \\ T\end{array}\right)^{\prime}$ deriving from $T^{\prime}=\bar{e}_{S \tau} \tau^{\prime}+\bar{e}_{S S} S^{\prime}$ and the relation $\tau^{\prime}=-\sigma^{-1} v^{\prime}$ obtained from (6.3), yielding, finally,

$$
\left(\begin{array}{l}
\tau \\
S
\end{array}\right)^{\prime}=\tau\left(\begin{array}{cc}
-\sigma & 0 \\
\bar{e}_{S \tau} & \bar{e}_{S S}
\end{array}\right)^{-1}\left(\begin{array}{cc}
\mu & 0 \\
\mu v & \kappa
\end{array}\right)^{-1}\left(\begin{array}{c}
-\sigma\left(v-v_{-}\right)+p-p_{-} \\
-\sigma\left(e+v^{2} / 2-e_{-}-v_{-}^{2} / 2\right)+p v-p_{-} v_{-}
\end{array}\right) .
$$

From $(\bar{\tau}, \bar{S})(x)$, we can recover all other variables through $(6.3)$ and the equation of state.

A final detail is that in the large-amplitude limit $S \rightarrow-\infty$, the traveling-wave ODE 6.5 features a wide separation of scales. Specifically, the linearized equations about endstates $U_{ \pm}$,

$$
\left(\begin{array}{c}
\tau \\
S
\end{array}\right)^{\prime}=\left(\begin{array}{cc}
-\frac{1}{\sigma} & 0 \\
\frac{\bar{e}}{\bar{e}_{S T}} & \frac{1}{\bar{e}_{S S}}
\end{array}\right)_{ \pm}\left(\begin{array}{cc}
\frac{1}{\mu} & 0 \\
-\frac{v}{\kappa} & \frac{1}{\kappa}
\end{array}\right)_{ \pm}\left(\begin{array}{cc}
\sigma^{2}+c^{2} & \bar{p}_{S} \\
v\left(2 \sigma^{2}-c^{2}\right) & -\sigma T+\bar{p}_{S} v
\end{array}\right)_{ \pm}\left(\begin{array}{c}
\tau \\
S
\end{array}\right)
$$

for global model (4.1) in the regime $1<<\left|S_{-}\right|<<C$ considered in the crucial stability transition regime, is asymptotic at $U_{-}(x \rightarrow-\infty)$ to (computing $|\sigma| \sim 1, c=-\bar{e}_{\tau \tau} \sim 1, \bar{e}_{S} \sim 1, \bar{e}_{S \tau} \sim C^{-1}$, $\left.\bar{e}_{S S} \sim C^{-2}\right)$

$$
\left(\begin{array}{c}
\tau \\
S
\end{array}\right)^{\prime} \sim\left(\begin{array}{cc}
\frac{2}{\mu} & 0 \\
-\frac{v_{-} C}{\kappa} & \frac{C^{2}}{\kappa}
\end{array}\right)\left(\begin{array}{c}
\tau \\
S
\end{array}\right)
$$

which exhibits growth modes with exponential rates $\sim 1 / \mu$ and $C^{2} / \kappa$ differing by two or more orders of magnitude. Similar but more extreme behavior is inherited by the local model (4.3) in the regime $\tau_{-} \rightarrow+\infty, S_{-} \sim \frac{1}{2}(3-\tau) \rightarrow-\infty$ roughly corresponding to $C \rightarrow \infty$ for 4.1 , with growth rates $\sim 1 / \mu$ and $\tau_{-} e^{\tau_{-} / 2} / \kappa$.

We find that this gives trouble for the standard method (described further in Appendix C.2) of solving for profiles with a boundary-value solver with projective boundary conditions; indeed, we are unable to compute by this method beyond approximately the range $|C| \leq 75$ for 4.1 or $\tau_{-} \leq 19$ for 4.3 .9

Above these values, we rely on a more primitive, shooting method, starting at a point distance $10^{-8}$ from the saddle $U_{+}$in the direction of the direction of the stable subspace of $(6.6)$, and

\footnotetext{
9 This is with initial guess given by a simple tanh interpolation. We can go slightly farther by continuing along the Hugoniot curve, using previous profile as initial guess; however, the computations are still extremely slow.
} 
integrating backward in $x$ the traveling-wave ODE (6.5) using an appropriate stiff numerical ODE solver until the solution comes within $10^{-8}$ of the repellor $U_{-}$. This simple solution gives excellent results for essentially arbitrary values of $S_{-}$.

6.1.2. Evans computations. In computing the Evans function, we face the same numerical stiffness issues that we faced for the profile equation (recall that at freqency $\lambda=0$, the eigenvalue equation reduces to the linearized traveling-wave ODE). Though it is standard practice for us to compute the Evans function by shooting, we find it necessary to carry out this shooting algorithm using a stiff ODE solver rather than the standard RK45. For further discussion, see Appendix C.2.

A related issue is that for $C>10$ or so in (4.1), or for $\tau_{-}>6$ in 4.3 , the standard Evans function experiences exponential growth/decay arising from spatial variation in the coefficient matrix of the Evans (i.e., first-order eigenvalue) system so great as to go out of numerical range- in this case, returning a value that is to numerical approximation identically zero. Similar phenomena have been seen to arise in other numerically difficult Evans computations [BZ1, BZ2, BHLyZ1. We follow the simple solution here (mentioned also in the cited references) of turning off growth; that is, computing using the polar coordinate method of [HuZ1], we suppress variation in the radius and simply evolve orthonormal bases of the subspaces of decaying solutions at plus and minus spatial infinity (the "polar no radial" setting in the STABLAB Evans package). This amounts to the continuous orthogonalization method of Drury [Dr], which preserves winding numbers/zeroes of the Evans function, but loses the desirable property of analyticity [Z4]. (It is in fact $C^{\infty}$ in $\lambda$.)

The same numerical stiffness issues prevent degrade our usual estimates excluding unstable eigenvalues outside a semicircle of radius $R$. In this section, therefore, we often compute for a fixed radius $R$ without guarantees that there are no unstable eigenvalues of larger modulus.

Remark 6.3. The presence of the very slowly decaying term $e^{S / C^{2}-\tau / C}$ in the Evans function coefficient arixing in our global counterexample (4.1) degrades the standard convergence estimates described in, e.g., [Z4, Z5], wich depends on uniform exponential convergence of the coefficient matrix of (C.7) to its limits as $x \rightarrow \pm \infty$. However, uniform-in- $C$ estimates are easily recovered similarly as (but much more simply than) in [HLZ], by the incorporation of an additional "tracking" argument in the slowly-varying far-field regime. A similar argument, though we do not carry it out here, may be used to rigorously verify convergence as $C \rightarrow \infty$ of the Evans function for the global counterexample (suitably normalized) to the Evans function for the local example of Section 3.1 .

6.2. Global counterexample. Returning to the equation of state

$$
\bar{e}(\tau, S)=\frac{e^{S}}{\tau}+C^{2} e^{S / C^{2}-\tau / C}, \quad C>1
$$

shown to exhibit inviscid instability in Theorem A, we report the results of our numerical Evans function investigations. In our study we examined the parameter set $S_{-}=\{-1,-1.1, \ldots,-50\}$, $\left(\tau_{+}, S_{+}\right)=(1,0)$, for $C=10$. We solved the profile using Matlab's boundary value solver bvp5c. In addition, we computed the Evans function on a smaller radius for $\left(\tau_{+}, S_{+}\right)=(1,0)$ and several values of $S_{-}$including $S_{-}=-10^{5}$ for $C=100$ with $R=4$, solving for the profile using Matlab's implicit ODE solver, ode15s.

The results of these investigations were, in all cases, that, as shock amplitude was increased (i.e., $S_{-}$decreased), there was, similarly as in the inviscid stability analysis, a single stability transition consisting of a real root of multiplicity one passing through the origin into the unstable complex halfplane $\Re \lambda>0$, where it remained thereafter as the only unstable eigenvalue. In particular, there were seen no complex unstable eigenvalues at any point in the process. That is, in this case, viscous and inviscid stability predictions appear to agree.

For $C=10$, the computed inviscid stability transition occurs approximately at $S_{-}=-23.2$, whereas the computed viscous stability transition occurs for $S_{-} \in(-22.1,-22.2)$; recall that, by 
the analytical theory, these two values should agree, as parameters for which a real root passes through the origin. Except for this difference in location of the transition point, the results of inviscid and viscous numerical stability analyses agreed. The relatively large size of this discrepancy we ascribe to the stiffness of the underlying eigenvalue equation both for viscous and inviscid cases; compare for example to the excellent agreement found in the investigations of the nonstiff system considered in Section 7 .

The study was further challenged by the slow rate at which the Evans function converges to its high frequency behavior $D(\lambda) \sim C_{1} e^{C_{2} \sqrt{\lambda}}$, as described in C.8), Appendix C.2. In tables 1 and 2 we provide data about curve fitting the Evans function with $C_{1} e^{C_{2} \sqrt{\lambda}}$ as $\lambda \rightarrow \infty$. As seen in the tables, the needed radius for convergence for large $C$ exceeds the range where numerical computations can be carried out accurately. Consequently, we arbitrarily chose $R=250$ for the Evans function study. Figure 6 demonstrates typical output from our study.

6.3. Local counterexample. Next, we turn to the local model (4.3) obtained by expansion of the global model in the $C \rightarrow \infty$ limit, describing the results of a corresponding numerical Evans function investigation in that case. In our study we examined the parameter set $S_{-}=\{-1,-1.1, \ldots,-8.1\} \backslash\{-3.3\}$. Numerically, we found that both the inviscid and viscous stability transitions occurred around $S_{-}=-10 / 3$. For all parameters covered in our study, the viscous Evans function had one root or no root according as the shock was inviscid unstable or stable. As with the global model, we could not carry out a numerical high frequency study, and so we arbitrarily chose $R=250$ for the contour radius in our Evans function computations. Figure 7 demonstrates typical output from our study. In conclusion, the results of our numerical study indicate in this case too that viscous and inviscid stability agree.

6.4. Stable example. Finally, we briefly discuss the case of example (4.6) seen to be inviscidly stable. In our study we examined the parameter set $S_{-} \in\{-0.1,-0.2, \ldots,-11.5\}$. For a few values the boundary value solver could not solve the profile equation, returning a "singular Jacobian"

\begin{tabular}{|c|c|c|c|}
\hline$R$ & error & $C_{1}$ & $C_{2}$ \\
\hline 2 & 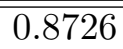 & $\overline{0.03233}$ & 2.427 \\
\hline 4 & 1.693 & 0.002496 & 2.996 \\
\hline 8 & 2.103 & $3.386 \mathrm{e}-05$ & 3.639 \\
\hline 16 & 6.33 & $3.659 \mathrm{e}-08$ & 4.281 \\
\hline 32 & 12.47 & $1.476 \mathrm{e}-12$ & 4.816 \\
\hline 64 & 17.38 & $1.784 \mathrm{e}-18$ & 5.108 \\
\hline 128 & 4.211 & $3.922 \mathrm{e}-26$ & 5.171 \\
\hline 256 & 0.9991 & $7.957 \mathrm{e}-36$ & 5.051 \\
\hline 512 & 0.9949 & $3.594 \mathrm{e}-48$ & 4.828 \\
\hline 1024 & 0.9994 & $8.181 \mathrm{e}-65$ & 4.611 \\
\hline 2048 & 0.9945 & $2.664 \mathrm{e}-88$ & 4.456 \\
\hline 4096 & 0.9805 & $5.719 \mathrm{e}-122$ & 4.362 \\
\hline 8192 & 0.948 & $3.166 \mathrm{e}-170$ & 4.312 \\
\hline $1.638 \mathrm{e}+04$ & & $\mathrm{NaN}$ & $\mathrm{NaN}$ \\
\hline
\end{tabular}

TABle 1. For $C=10, S_{-}=-15$ in the global model we compare how well $C_{1} e^{C_{2} \sqrt{\lambda}}$ approximates $D(\lambda)$ for $|\lambda|=R$. Here $R$ is the radius of the semicircle on which we compute the Evans function, $C_{1}$ and $C_{2}$ are the curve fit returned, and the convergence error is the maximum relative error between $D(\lambda)$ and $C_{1} e^{C_{2} \sqrt{\lambda}}$ evaluated at $\lambda=R, R i$. 
(a)

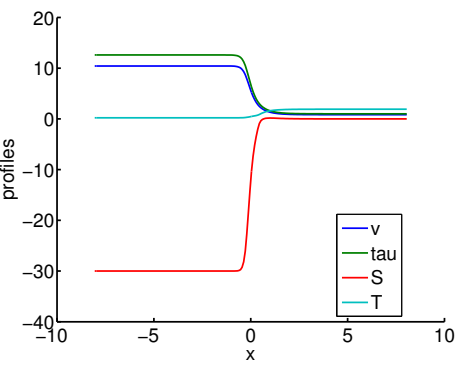

(b)

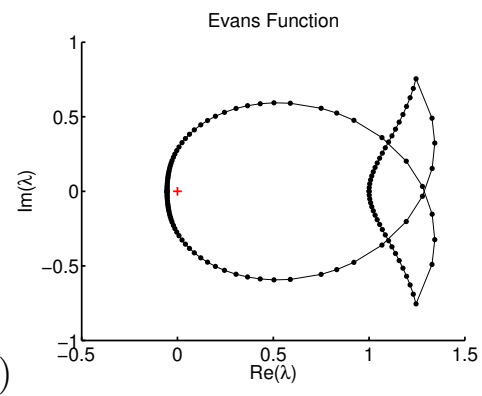

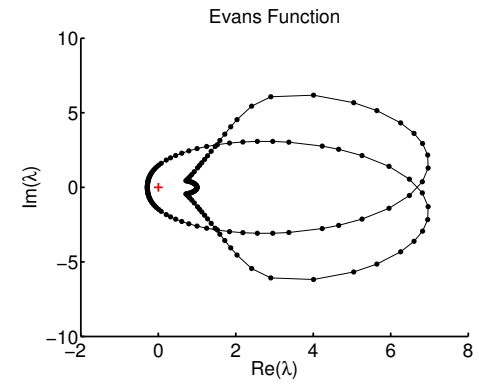

Figure 6. Viscous study results for $\bar{e}(S, \tau)=e^{S} / \tau+C^{2} e^{C^{-1}\left(C^{-1} S-\tau\right)}$ with $S_{-}=$ $-30, C=10, \mu=\kappa=1,\left(\tau_{0}, S_{0}\right)=(1,0)$. (a) Viscous profile. (b) Integrated Evans function computed with the adjoint polar coordinate method, evaluated on a semi-circle with radius $R=1$. Winding number is 1. (c) As in (b) but with $R=250$.

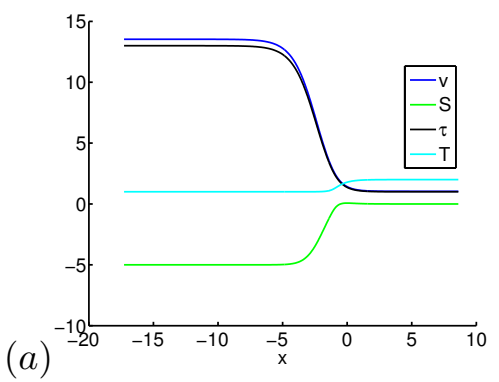

(b)

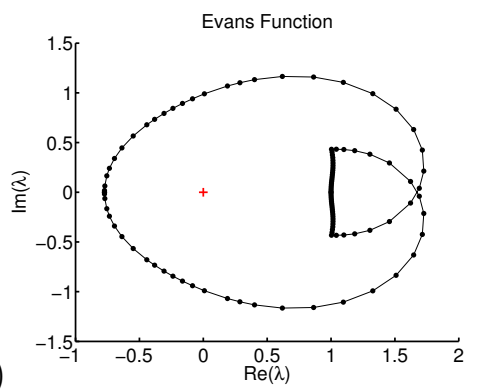

(c)

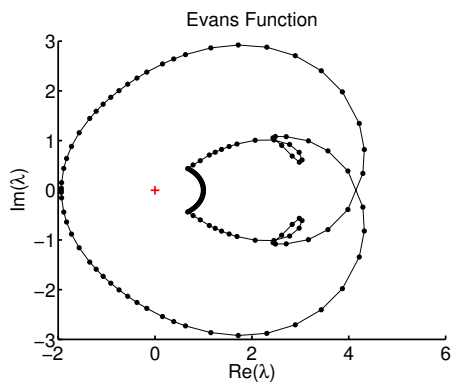

Figure 7. Viscous study results for $\bar{e}(S, \tau)=e^{S} / \tau+S+\tau^{2} / 2, S_{-}=-5, \mu=\kappa=1$, $\left(\tau_{0}, S_{0}\right)=(1,0)$. (a) Viscous profile. (b) Integrated Evans function computed with the adjoint polar coordinate method, evaluated on a semi-circle with radius $R=1$. Winding number is 1 . (c) As in (b) but with $R=250$.

error. For these, we solved the profile using MATLAB's implicit ODE solver, ode15s. To ensure that we computed the Evans function on a contour enclosing any possible unstable eigenvalues, we performed a high-frequency study curve-fitting with $C_{1} e^{C_{2} \sqrt{\lambda}}$, requiring that relative error be no

\begin{tabular}{|c|c|c|c|}
\hline$R$ & error & $C_{1}$ & $C_{2}$ \\
\hline \hline 2 & 0.3373 & 0.711 & 0.2412 \\
4 & 0.3457 & 0.9109 & 0.04668 \\
8 & 0.287 & 1.268 & -0.08408 \\
16 & 0.2102 & 1.876 & -0.1572 \\
32 & 0.1479 & 3.003 & -0.1944 \\
64 & 0.1055 & 5.439 & -0.2117 \\
128 & 0.0779 & 12.07 & -0.2202 \\
256 & 0.05837 & 36.77 & -0.2253 \\
\hline
\end{tabular}

TABle 2. For $C=2$ in the global model we compare how well $C_{1} e^{C_{2} \sqrt{\lambda}}$ approximates $D(\lambda)$ for $|\lambda|=R$. Here $R$ is the radius of the semicircle on which we compute the Evans function, $C_{1}$ and $C_{2}$ are the curve fit returned, and the convergence error is the maximum relative error between $D(\lambda)$ and $C_{1} e^{C_{2} \sqrt{\lambda}}$ evaluated at $\lambda=R, R i$. 
greater than 0.2 between the Evans function and the approximating function. Table 3 demonstrates high-frequency convergence for a typical parameter. In addition we used an adaptive $\lambda$ mesh to ensure that the change in argument was no greater than 0.2 between successive $\lambda$ points. We found for all parameters studied that the Evans function has no root in the right half complex plane, consistent with stability. The maximum relative change in $\lambda$ for each contour computed was 0.1005. The average value of $R$ was 4 and the average number of points computed was 40 (80 points after using conjugate symmetry of the Evans function to reflect the values computed on the upper half-plane $\Im \lambda \geq 0$ to the lower half-plane). The average time to solve a profile was 12.8 seconds and the average time to solve the Evans function was 6.76 seconds using 8 cores on a Mac Pro. Evans function output for a typical point in parameter space is given in Figure 8, In all cases, we found a winding number of zero, indicating viscous stability in agreement with our inviscid findings.
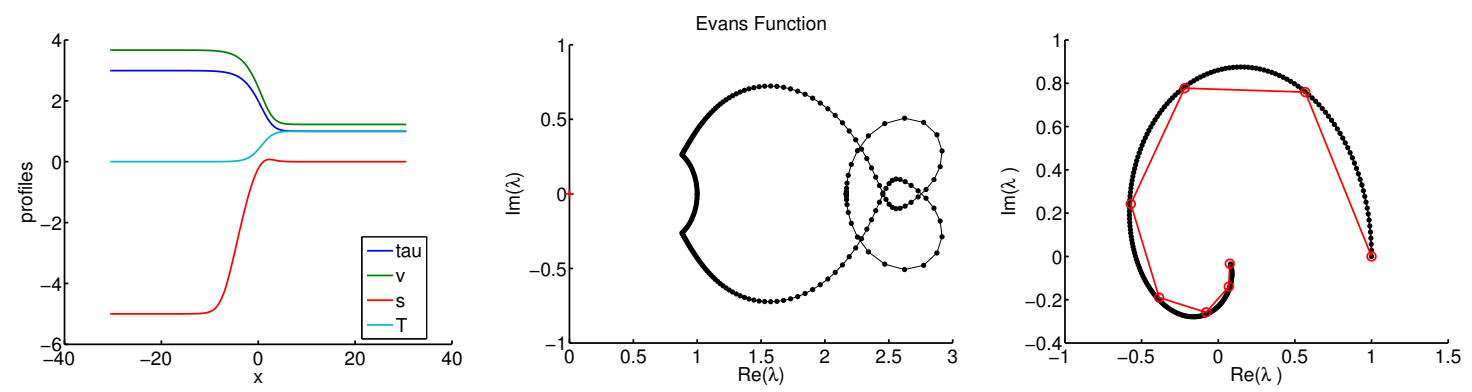

Figure 8. Viscous study results for $\bar{e}(S, \tau)=e^{S} / \tau+\tau^{2} / 2$ with $\left(\tau_{0}, S_{0}\right)=(1,0)$, and $S_{-}=-5$. (a) Viscous profile. (b)Integrated Evans function computed with the adjoint polar coordinate method, evaluated on a semi-circle with radius $R=250$. Winding number is 1 . (c) The Evans function evaluated at 8 points along a semicircle of radius $\mathrm{R}=256$ is plotted with open circles. The best curve fit of the Evans function with $C_{1} e^{C_{2} \sqrt{\lambda}}$ is plotted with closed dots. The relative error between the two is 0.069 .

\begin{tabular}{|c|c|c|c|}
\hline$R$ & error & $C_{1}$ & $C_{2}$ \\
\hline \hline 2 & 0.04501 & 0.6019 & 0.359 \\
4 & 0.08243 & 0.4554 & 0.3933 \\
8 & 0.1244 & 0.2953 & 0.4312 \\
16 & 0.147 & 0.1556 & 0.4651 \\
32 & 0.1416 & 0.06249 & 0.4901 \\
64 & 0.1179 & 0.01757 & 0.5052 \\
128 & 0.09174 & 0.002994 & 0.5136 \\
256 & 0.06888 & 0.0002471 & 0.5191 \\
512 & 0.05042 & $7.366 \mathrm{e}-06$ & 0.5223 \\
\hline
\end{tabular}

TABLE 3. For $S_{-}=-5$ in the stable model we compare how well $C_{1} e^{C_{2} \sqrt{\lambda}}$ approximates $D(\lambda)$ for $|\lambda|=R$. Here $R$ is the radius of the semicircle on which we compute the Evans function, $C_{1}$ and $C_{2}$ are the curve fit returned, and the convergence error is the maximum relative error between $D(\lambda)$ and $C_{1} e^{C_{2} \sqrt{\lambda}}$ evaluated at $\lambda=R, R i$. 


\section{Designer systems}

We conclude by investigating the range of possible behaviors for general systems possessing a convex entropy. Let $A(x)$ be a given real symmetric $n \times n$ matrix-valued function, and $\mathcal{B}(x)$ a given real positive definite $n \times n$ matrix-valued function, in the sense that $\Re \mathcal{B}:=\frac{1}{2}\left(\mathcal{B}+\mathcal{B}^{T}\right)>0$, each converging exponentially as $x \rightarrow \pm \infty$ to limits $\mathcal{A}_{ \pm}$and $\mathcal{B}_{ \pm}$, and consider the eigenvalue problem

$$
\lambda u=\partial_{x}\left(\mathcal{B}(x) \partial_{x}-\mathcal{A}(x)\right) u, \quad u \in \mathbb{C}^{n},
$$

resembling those found in the stability analysis of viscous shock waves. This might be considered as a model for the type of eigenvalue equation that would arise from a symmetrizable system, in particular a system possessing a convex entropy.

Our first observation is that, by an ingenious construction of Bianchini $[\mathrm{B}$, any such system can in fact be realized as the eigenvalue problem for a viscous shock wave solution of a system of conservation laws with a viscosity-compatible strictly convex entropy. Specifically, consider the system

$$
\begin{aligned}
u_{t}+(A(v) u)_{x} & =\left(B(v) u_{x}\right)_{x}, \\
v_{t}+\left(\frac{1}{2} u \cdot A^{\prime}(v) u+\frac{1}{2} v^{2}\right)_{x} & =v_{x x},
\end{aligned}
$$

where $v \in \mathbb{R}^{1}$.

This contains a trivial standing viscous shock solution $(\bar{u}, \bar{v})=(0, \bar{v}(x))$, where $\bar{v}(x):=-\tanh (x / 2)$ is a shock of Burgers equation $v_{t}+\left(v^{2} / 2\right)_{x}=v_{x x}$. Linearizing (7.2) about $(\bar{u}, \bar{v})$, we obtain

$$
\left(\begin{array}{l}
u \\
v
\end{array}\right)_{t}+\left(\begin{array}{cc}
A(\bar{v}) & 0 \\
0 & \bar{v}
\end{array}\right)\left(\begin{array}{l}
u \\
v
\end{array}\right)_{x}=\left(\left(\begin{array}{cc}
B(\bar{v}) & 0 \\
0 & 1
\end{array}\right)\left(\begin{array}{l}
u \\
v
\end{array}\right)_{x}\right)_{x}
$$

which decouples into a (stable) linearized Burgers equation in $v$ and an equation

$$
u_{t}+(A(\bar{v}(x)) u)_{x}=\left(B(\bar{v}(x)) u_{x}\right)_{x},
$$

that has the associated eigenvalue equation (7.1) provided that we choose $A(v):=\mathcal{A}(2 \arctan (-v))$ and $B(v):=\mathcal{B}(2 \arctan (-v))$. Moreover, it is easily verified that $(7.2$ possesses a viscositycompatible stricly convex entropy $\eta(u, v):=\frac{1}{2}|u|^{2}+\frac{1}{2}|v|^{2}$, with associated entropy flux $q(u, v):=$ $\frac{1}{2}\left\langle u, A((v) u\rangle+\frac{1}{3}|v|^{3}+\frac{1}{2} v\left\langle u, A^{\prime}(v) u\right.\right.$. That is, $d \eta d F=d q$ and $d^{2} \eta\left(\begin{array}{cc}B & 0 \\ 0 & 1\end{array}\right)=\left(\begin{array}{cc}B & 0 \\ 0 & 1\end{array}\right)$ is positive definite, where $F(u, v)=\left(\begin{array}{c}A(v) u \\ \frac{1}{2} u \cdot A^{\prime}(v) u+\frac{1}{2} v^{2}\end{array}\right)$. This means that we may search for stability phenomena of symmetrizable systems possessing a viscosity-compatible strictly convex entropy, by considering the linear problem (7.1), which is free to our specifications.

7.1. The rotating model. A natural choice in seeking examples of instability is the rotating model consisting of $(7.2)$ with

$$
A(v)=R_{\theta(v)} A_{m} R_{-\theta(v)}, \quad B(v) \equiv \mathrm{Id},
$$

where $R_{\theta}:=\left(\begin{array}{cc}\cos \theta & -\sin \theta \\ \sin \theta & \cos \theta\end{array}\right), A_{m}=\left(\begin{array}{cc}1 & 0 \\ 0 & -1\end{array}\right)$, and

$$
\theta(v)=M \pi v
$$

$M$ an arbitrary real number, considering the family of stationary shocks $\bar{v}_{\gamma}(x):=-\gamma \tanh (\gamma x / 2)$.

For, it is readily seen that these are Lax 2-shocks, with a Lopatinski determinant

$$
\delta_{\gamma, M}=\operatorname{det}\left(\begin{array}{ccc}
\cos -M \pi \gamma & -\sin (M \pi \gamma) & 0 \\
\sin -M \pi \gamma & \cos (M \pi \gamma) & 0 \\
0 & 0 & -2 \gamma
\end{array}\right)=-2 \gamma\left(\cos ^{2}(M \pi \gamma)-\sin ^{2}(M \pi \gamma)\right)
$$


that changes sign from negative to positive and back infinitely often as $\gamma$ increases from the smallamplitude limit $\gamma=0$, with the first stability transition occuring at $M \pi \gamma_{*}=\pi / 4$, or $\gamma_{*}=1 / 4 M$. This in passing gives another example of a $3 \times 3$ system with convex entropy exhibiting inviscidly unstable shock waves,

7.1.1. Evans system. For profile $\bar{u} \equiv 0, \bar{v}=-\gamma \tanh (\gamma x / 2)$, the integrated Evans system for the decoupled, $u$ equation $(7.4)$ is

$$
W^{\prime}=\mathcal{A}(x, \lambda) W, \quad \mathcal{A}=\left(\begin{array}{cc}
0 & I \\
\lambda B^{-1} & B^{-1} A
\end{array}\right)(\bar{v}(x)) .
$$

Remark 7.1. Setting $W=T Y$, where $T:=\left(\begin{array}{cc}R_{\theta} & 0 \\ 0 & R_{\theta}\end{array}\right)$, we may convert (7.7) to $Y^{\prime}=\mathbb{B} Y$, where

$$
\mathbb{B}=T^{-1} \mathbb{A} T-T^{-1} T^{\prime}=\left(\begin{array}{cc}
0 & I \\
\lambda & A_{m}
\end{array}\right)-M \pi \bar{v}^{\prime}(x)\left(\begin{array}{cc}
J & 0 \\
0 & J
\end{array}\right),
$$

and $J:=\left(\begin{array}{cc}0 & -1 \\ 1 & 0\end{array}\right)$. Involving only exponential functions in $x$ entering through the scalar multiplier $\bar{v}^{\prime}$, this seems possibly amenable to exact solution, an interesting direction for further study.

7.1.2. Transversality of profiles. Again appealing to the triangular form of the equations, we find that transversality of profiles is equivalent to nonexistence of asymptotically decaying solutions of the $u$-component $u^{\prime}=A(\bar{v}(x)) u$ of the linearized standing-wave equation. More, the transversality coefficient $\nu$, like the Lopatinski determinant, factors into $-2 \gamma$ times a transversality coefficient for this decoupled $u$-component.

Lemma 7.2. For $|M \gamma|$ bounded, profiles are transversal for $\gamma$ sufficiently small. More, when appropriately normalized, (i) $\nu /(-2 \gamma) \rightarrow 1$ as $\gamma \rightarrow 0^{+}$and (ii) $\nu /(-2 \gamma) \rightarrow \delta /(-2 \gamma)$ as $\gamma \rightarrow+\infty$, while (iii) $\nu /(-2 \gamma)$ changes sign at least $[4(M \gamma-1)]$ times as $\gamma$ increases from $0^{+}$to $+\infty$, where $[\cdot]$ denotes the greatest integer function.

Proof. (i) (Basic tracking argument; see [Z5].) Setting $u=R_{\theta} z$, similarly as in Remark 7.1, we obtain

$$
z^{\prime}=A_{m} z+M \bar{v}^{\prime}(x) J, \text { with } J:=\left(\begin{array}{cc}
0 & -1 \\
1 & 0
\end{array}\right) .
$$

Observing that $\left|M \bar{v}^{\prime}(x)\right| \leq M \gamma^{2} \leq C \gamma \rightarrow 0$ for $\gamma \rightarrow 0$ and $|M \gamma| \leq C$, we obtain the result by the Tracking Lemma of [ZH], a standard estimate for slowly-varying-coefficient systems, after factoring out the (real) exponential growth rates in growing and decaying modes.

(ii) Noting, for (7.5)-(7.6) that Evans system (7.7) satisfies $\left|\mathcal{A}(x)-\mathcal{A}_{ \pm}\right| \leq C M \gamma e^{-\gamma|x|} \leq C_{2} e^{-\gamma|x|}$ for $x \gtrless 0$, we obtain by the Convergence Lemma of [PZ] that the Wronskian $\nu$ converges up to an error of order $\left\|C_{2} e^{-\gamma|x|}\right\|_{L^{1}}=O(C / \gamma) \rightarrow 0$ to the determinant of a (smoothly chosen real) stable eigenvector of $\mathcal{A}_{+}$and a (smoothly chosen real) unstable eigenvector of $\mathcal{A}_{-}$, i.e., $\delta /(-2 \gamma)$.

(iii) Reviewing the previous arguments more closely, we find that for $\gamma$ sufficiently small the decaying mode as $x \rightarrow-\infty$ rotates angle $2 M \gamma \pi$, while for $\gamma$ sufficiently large it rotates at most $\pi$, with the directions of stable and unstable subspaces as $x \rightarrow \pm \infty$ held fixed for all $\gamma$. Thus, as $\gamma$ goes from 0 to $+\infty$, the decaying mode as $x \rightarrow \infty$ crosses the decaying mode as $x \rightarrow+\infty$ at least $[4(M \gamma-1)]$ times, each time signaling an associated change in sign.

From Lemma 7.2 , we find that, fixing $K$ sufficiently large, taking $\gamma_{*}$ sufficiently small and $M:=K / \gamma_{*}$, and varying $0 \leq \gamma \leq \gamma_{*}$, the profiles $\bar{v}_{\gamma}$ remain transversal, while the Lopatinski determinant changes sign $\approx 2 K$ times, signaling (recall (5.6)) $2 K$ passages of an eigenvalue through the imaginary axis, hence appearance of up to $2 K$ unstable roots. (Our numerical experiments indicate that these roots all cross in one direction, similarly as in standard Sturm-Liouville theory.) 
On the other hand, fixing $M \gamma=$ constant, and increasing $\gamma$ from $0^{+}$, we find that $\delta$ stays constant, while $\nu$ changes sign at least $\approx 4(M \gamma-1)$ times. More, varying $M \gamma$ within a bounded set for $\gamma$ sufficiently large, we find that changes of sign in $\delta$ and $\nu$ may be made to occur arbitrarily close together, increasing the chance of collision of roots and subsequent splitting into a complex conjugate pair. (Recall that changes of sign of $\delta$ and $\nu$ signal crossings through the origin of roots of the Evans function $D$.) This simple rule of thumb guides our strategy in searching for complex roots and Hopf bifurcation, and indeed gives a reasonable a posteriori fit to the data that we see. Why some colliding roots split into the complex plane while others appear to stay real is an interesting question to which we do not have an answer.

7.1.3. High-frequency bounds. We start by bounding the modulus of unstable eigenvalues.

Lemma 7.3. For system (7.3) with the choices (7.5)-(7.6), there are no unstable eigenvalues with modulus $|\lambda| \geq 4$.

Proof. Let $A$ be as in 7.5 and consider the integrated eigenvalue problem, $\lambda u+A u^{\prime}=u^{\prime \prime}$. Multiplying by the conjugate $\bar{u}$ and integrating over $\mathbb{R}$ yields

$$
\lambda\left\|u_{j}\right\|^{2}+\int_{\mathbb{R}} a_{j 1} \bar{u}_{j} u_{1}^{\prime} d x+\int_{\mathbb{R}} a_{j 2} \bar{u}_{j} u_{2}^{\prime} d x=\int_{\mathbb{R}} \bar{u}_{1} u_{1}^{\prime \prime} d x, \quad j=1,2,
$$

where $a_{j i}$ is the $j$-i entry of $A$. Integrating the righthand side by parts and rearranging gives

$$
\lambda\left\|u_{j}\right\|^{2}+\left\|u_{j}^{\prime}\right\|^{2}=-\int_{\mathbb{R}} a_{j 1} \bar{u}_{j} u_{1}^{\prime} d x-\int_{\mathbb{R}} a_{j 2} \bar{u}_{j} u_{2}^{\prime} d x, j=1,2 .
$$

Taking the real part of 7.10 for $\Re(\lambda) \geq 0$, and applying Cauchy's inequality, we have

$$
\Re(\lambda)\left\|u_{j}\right\|^{2}+\left\|u_{j}^{\prime}\right\|^{2} \leq C\left(\int_{\mathbb{R}}\left|u_{j}\left\|u_{1}^{\prime}\left|d x+\int_{\mathbb{R}}\right| u_{j}\right\| u_{2}^{\prime}\right| d x\right) \leq C\left(2 \varepsilon\left\|u_{j}\right\|^{2}+\frac{1}{4 \varepsilon}\left\|u_{1}^{\prime}\right\|^{2}+\frac{1}{4 \varepsilon}\left\|u_{2}^{\prime}\right\|^{2}\right),
$$

for $j=1,2$ where (using $\sin 2 \theta=2 \cos \theta \sin \theta) C=\max _{x \in \mathbb{R}}\left|a_{j, i}(x)\right|=1$. Similarly,

$$
|\Im(\lambda)|\left\|u_{j}\right\|^{2} \leq C\left(2 \varepsilon\left\|u_{j}\right\|^{2}+\frac{1}{4 \varepsilon}\left\|u_{1}^{\prime}\right\|^{2}+\frac{1}{4 \varepsilon}\left\|u_{2}^{\prime}\right\|^{2}\right),
$$

Summing (7.11) and (7.12) for $j=1,2$ we have

$$
(\Re(\lambda)+|\Im(\lambda)|)\left(\left\|u_{1}\right\|^{2}+\left\|u_{2}\right\|^{2}\right)+\left\|u_{1}^{\prime}\right\|^{2}+\left\|u_{2}^{\prime}\right\|^{2} \leq C\left(4 \varepsilon|| u_{1}\left\|^{2}+4 \varepsilon\right\| u_{2}\left\|^{2}+\frac{1}{\varepsilon}\right\| u_{1}^{\prime}\left\|^{2}+\frac{1}{\varepsilon}\right\| u_{2}^{\prime} \|^{2}\right) .
$$

Taking $\varepsilon=C$, we obtain $(\Re(\lambda)+|\Im(\lambda)|)\left(\left\|u_{1}\right\|^{2}+\left\|u_{2}\right\|^{2}\right) \leq 4 C^{2}\left(\left\|u_{1}\right\|^{2}+\left\|u_{2}\right\|^{2}\right)$, so that for $\Re(\lambda) \geq 0,|\lambda| \leq 4 C^{2} \leq 4$.

7.2. Numerical results. Here we describe our numerical studies for the designer system (7.2).

7.2.1. Comparison of $D(0), \nu, \delta$. For system (7.7) with $\lambda=0$, explicit orthonormal initializing bases $R_{ \pm}$for the Evans function, i.e., bases for the stable (unstable) subspaces of $\mathcal{A}(-\infty)(\mathcal{A}(\infty))$, are given by

$$
R_{-}=\left\{\frac{1}{2}\left(\begin{array}{c}
\cos (M \gamma \pi) \\
\sin (M \gamma \pi) \\
\cos (M \gamma \pi) \\
\sin (M \gamma \pi)
\end{array}\right),\left(\begin{array}{c}
-\sin (M \gamma \pi) \\
\cos (M \gamma \pi) \\
0 \\
0
\end{array}\right)\right\}_{34}, \quad R_{+}=\left\{\frac{1}{2}\left(\begin{array}{c}
\sin (M \gamma \pi) \\
\cos (M \gamma \pi) \\
-\sin (M \gamma \pi) \\
-\cos (M \gamma \pi)
\end{array}\right),\left(\begin{array}{c}
\cos (M \gamma \pi) \\
-\sin (M \gamma \pi) \\
0 \\
0
\end{array}\right)\right\} .
$$


Likewise, initializing bases $r_{ \pm}$for the linearized traveling-wave ODE $w^{\prime}=A w$ of the unstable (stable) subspaces of $A( \pm \infty)$ are given by $r_{-}=\frac{1}{2}(\sin (M \gamma \pi))$, and $r_{+}=\frac{1}{2}\left(\begin{array}{l}-\sin (M \gamma \pi) \\ -\cos (M \gamma \pi)\end{array}\right)$. We compute $D(0)$ and $\nu$ with these choices, for which $(5.6)$ becomes essentially a tautology ${ }^{10}$

Figure 9 demonstrates the effects of the rotation in solving the Evans function ODE.

(a)

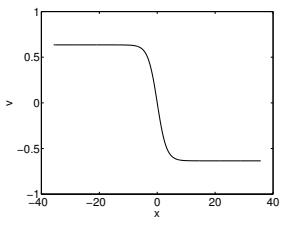

(b)

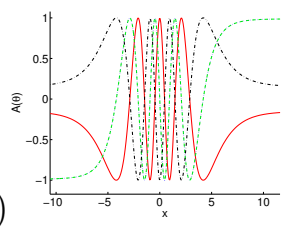

(c)

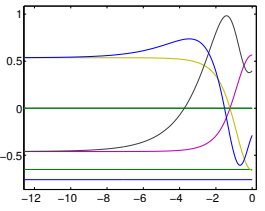

$(d)$

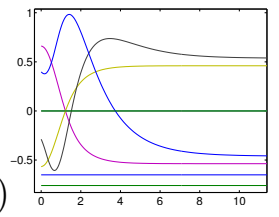

Figure 9. Rotating model with $M=2.7174, \gamma=0.635$. (a) Plot of profile $\bar{v}_{\gamma}(x)=$ $-\gamma \tanh (\gamma x / 2)$ against $x$. (b) Zoomed in plot of components of $A(\theta(x))$ against $x$. (c)-(d) Zoomed in plot of components of the evolved manifolds in the Evans function computations for $\lambda=27 / 2$.

$(a)$

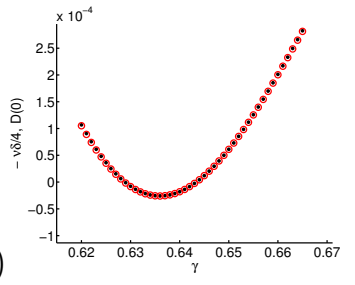

(b)

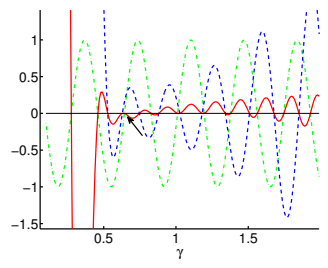

(c)

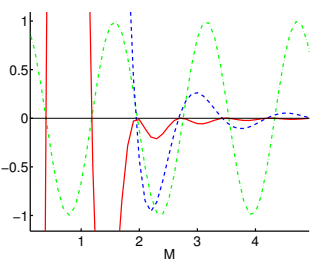

$(d)$

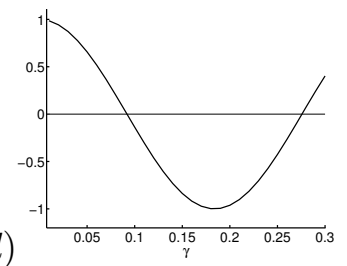

Figure 10. Rotating model. (a) Plot of $D(0)$ (solid black dots) and $-\delta \nu / 4$ (red circles) against $\gamma$ for $M=2.72$ demonstrating the identity $D(0)=-\nu \delta / 4$. (b) Plot of $D(0)$ (solid red), $\delta$ (dot-dashed green), and $\nu$ (dashed blue) against $\gamma$ for $M=2.72$. We mark with an arrow the general region where the Hopf bifurcation occurs. (c) Plot of $D(0)$ (solid red), $\delta$ (dot-dashed green), and $\nu$ (dashed blue) against $M$ with $\gamma=0.635$. (d) Plot of $\delta(\gamma)$ for $M=2.72$. Note that the first root of $\delta$ occurs at $\delta_{*}=1 /(4 M) \approx 0.09$.

7.2.2. Evans function computations. We now detail the results of our numerical study for the designer model. For all our Evans function computations we used the integrated form 7.7 of the eigenvalue problem. We used MATLABs implicit ODE solver ode15s with relative and absolute error bounds set respectively to $1 \mathrm{e}-6$ and $1 \mathrm{e}-8$. We used winding number computations and a divide and conquer scheme described in C.2 to find roots of the Evans function. To obtain a global picture of roots, we used Lemma 7.3 to ensure that the radius of the contour on which the Evans function is computed was sufficiently large to enclose all possible unstable eigenvalues. We also sometimes used a smaller radius for speed when looking just for the existence of Hopf bifurcations.

To search for complex roots and or Hopf bifurcation, we held fixed one of the parameters $\gamma, M$, and $M \gamma$ while letting a second parameter vary, examining the resulting motion of eigenvalues along these curves. The results of such experiments can be fairly complicated, and can involve a large number of roots (up to 16 in the experiment of Figure 11(b)). However, most of these roots are real for most of the time, and, when they do collide and split into a complex pair, tend quickly to rejoin

\footnotetext{
${ }^{10}$ Note that vectors $(*, *, 0,0)^{T}$ are stationary solutions of 7.7$)$, so that the Wronskian of the associated modes simplifies to $\delta$ times a $2 \times 2$ block involving third and fourth components, i.e., the Wronskian $\nu$ of $w^{\prime}=A w$.
} 
and become again real. Thus, to find complex roots, and in particular to identify Hopf bifurcations (roots that are not only complex, but cross the imaginary axis as a conjugate pair) involves finding a rather small-measure subset of parameter space, and requires a systematic search; they were not easy to find! An approach that proved quite useful in identifying Hopf bifurcations was to count the number of unstable roots at a selection of mesh points in the $\gamma-M \gamma$ plane and look for jumps of 2 across boundaries of different regions in the number of roots, then check with a refined study that (as generically should hold) these correspond to crossing of the imaginary axis by a non-real conjugate pair.

We note in Figure 10 that for $M=2.72$ the first root of $\delta(\gamma)$ occurs at $\delta_{*}=1 /(4 M) \approx 0.09$. Figure 11 (a) demonstrates that Hopf bifurcations exist and occur roughly periodically in the parameter $M \gamma$, with $\gamma$ near special values. We used $R=16$ for the contour radius and checked that $\delta(\gamma)$ had no roots in the vicinity of the jumps by 2 to verify that indeed they correspond to Hopf bifurcations. Figure 11 (b) shows that for $M \gamma$ fixed the number of Hopf bifurcations occurring increases as $\gamma \rightarrow 0$. Figure 11 (c) shows a Hopf bifurcation occurring as $M$ increases, for $\gamma$ fixed. Figure 12 (c) shows a Hopf bifurcation occurring as $\gamma$ increases, for $M$ fixed. (The latter corresponds to variation in shock amplitude for a fixed set of equations, hence is our true interest.) Figure 12 (d) shows crossing of the two eigenvalues with largest real part, with a double-multiplicity eigenvalue occurring at the point of crossing, violating simplicity of the top eigenvalue.

$(a)$

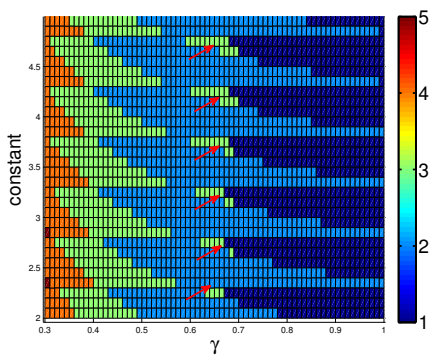

(b)

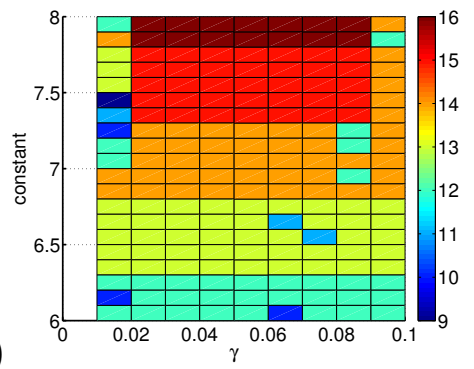

$(c)$

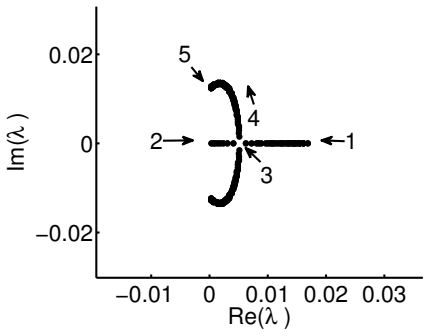

Figure 11. Designer system. (a) Color plot of the number of roots present for $(\gamma, M \gamma=$ constant $)$. Arrows indicate where the number of roots jumps by 2 corresponding to a Hopf bifurcation. (b) Color plot of the number of roots present for $(\gamma, M \gamma=$ constant). (c) Plot in the complex plane of the path two roots of the Evans function take as they cross the imaginary axis. Here $\gamma=0.65$ is fixed and $M$ varies. There is a root at about $\lambda=0.09$ not in the viewing window. (1) When $M=2.57$ there is one root in the viewing window traveling left, (2) at $M=2.5815$ a root passes through the origin traveling right, (3) the two roots collide at $M \approx 2.585$, (4) upon collision, the roots split off of the real axis, $(5)$ at $M \approx 2.661$ the roots pass through the imaginary axis.

Remark 7.4. Vanishing of $\nu$ appears to be closely related to both Hopf bifurcation and appearance of complex roots; hence, unlike the situation for gas dynamics, we have evidently violation of transversality of profiles within the parameter regime under study.

Acknowledgement: The numerical Evans function computations performed in this paper were carried out using STABLAB [BHZ2], a MATLAB-based numerical stability package developed by Jeffrey Humpherys together with Barker and Zumbrun. We gratefully acknowledge his contribution. Thanks also to Stefano Bianchini, Fedja Nazarov, and Ben Texier for interesting conversations on this topic, and in particular to Bianchini for his contribution through system (7.2) [Bi]. The second 
(a)

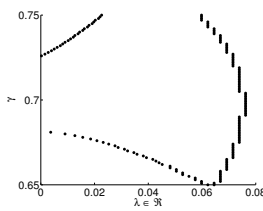

(b)

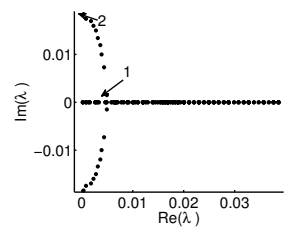

(c)

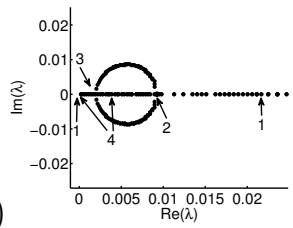

$(d)$

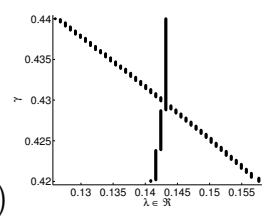

FiguRE 12. Designer system. In plots (a)-(c) there is a real root to the right of the viewing window. (a) Plot of $\gamma$ against root location for $M=3.1$. The two small modulus roots of the Evans function do not collide and split in the right half plane, but presumably they do in the left half-plane. (b) For $M=3.2836$ two roots of the Evans function collide and split into a non real complex conjugate pair which cross the imaginary axis indicating a Hopf bifurcation. The arrows correspond to the location of the roots of the Evans function for the following values of $\gamma,(1)$ $\gamma \approx 0.6545,(2) \gamma \approx 0.664$. (c) For $M=3.51$ two roots collide and split into a non real complex conjugate pair, but they rejoin in the right half plane. The arrows correspond to the location of roots for the following values of $\gamma,(1) \gamma \approx 0.6252,(2)$ $\gamma \approx 0.629,(3) \gamma \approx 0.6409$, (4) $\gamma=0.641$. (d) Plot of the location of the two largest modulus roots of the Evans function against $\gamma$ for $M=3.2836$. Note that the roots pass through each other.

(a)

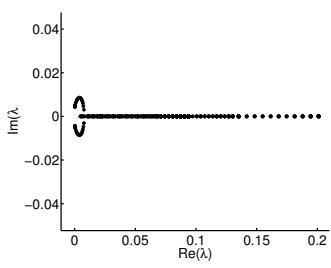

$(d)$

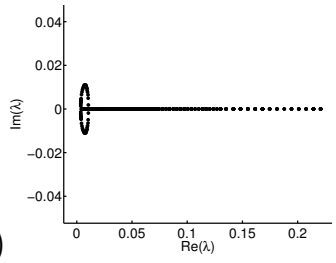

(g)

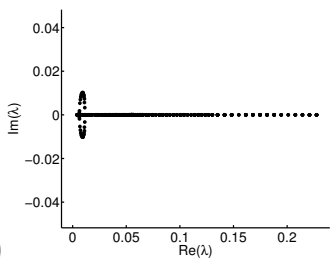

(b)

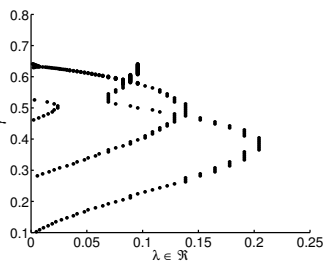

(e)

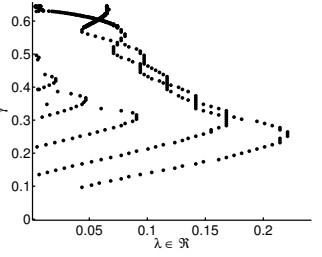

$(h)$

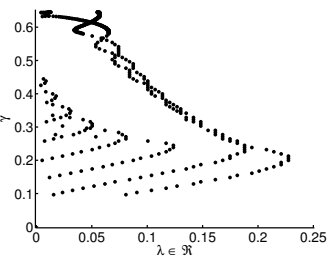

(c)

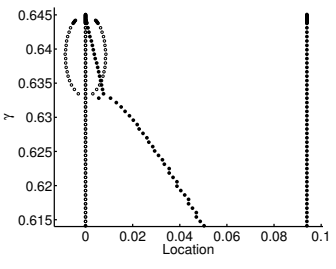

$(f)$

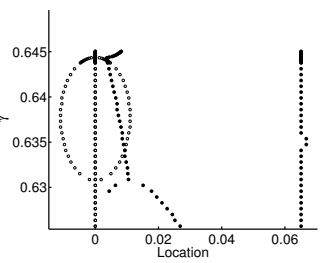

(i)

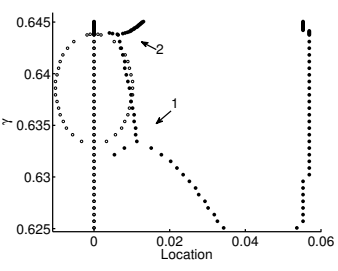

Figure 13. For (a)-(c), $M \approx 2.730$. For (d)-(f), $M \approx 5.8194$, and for (g)-(i), $M \approx 8.9230$. We have in (a), (d), and (g) plots of roots in the complex plane as $\gamma$ varies. In (b), (e), and (h) we plot $\gamma$ against location. In (c), (f), and (i) we plot $\gamma$ against location where a closed dot corresponds to the real part of the root and an open circle corresponds to the imaginary part. In (i) arrows 1 and 2 emphasize respectively where two roots on the real axis collide and split to form a complex conjugate pair and then collide again and split along the real axis, similarly as in (c) and (f). There are 3 roots in (a)-(c), 4 in (d)-(f), and 5 in (g)-(i). 
author thanks the University of Indiana, Bloomington and the third author thanks the École Normale Supérieure, Paris, the University of Paris 13, and the Foundation Sciences Mathématiques de Paris for their hospitality during visits in which this work was partially carried out. Thanks finally to Indiana University University Information Technology Services (UITS) for providing the QUARRY supercomputing environment used in our large-scale parallel Evans computations.

\section{Appendix A. Lopatinski computations for partial EQUATion of STATE}

To compute the Lopatinski condition in terms of the more general relation $p=\hat{p}(\tau, e)$, not necessarily connected with a full equation of state, rewrite 2.8 for smooth solutions as

$$
\begin{aligned}
\tau_{t}-v_{x} & =0, \\
v_{t}+p_{x} & =0, \\
e_{t}+p v_{x} & =0,
\end{aligned}
$$

so that, for $w=(\tau, v, e)^{T}, A_{ \pm}=\left(\begin{array}{ccc}0 & -1 & 0 \\ \hat{p}_{\tau} & 0 & \hat{p}_{e} \\ 0 & p & 0\end{array}\right)_{ \pm}, a_{1}=-c, a_{2}=0, a_{3}=c, c=\sqrt{-\hat{p}_{\tau}+p \hat{p}_{e}}$, and

$$
r_{1}^{+}=\left(\begin{array}{c}
1 \\
c \\
-p
\end{array}\right), \quad r_{2}^{+}=\left(\begin{array}{c}
-\hat{p}_{e} \\
0 \\
\hat{p}_{\tau}
\end{array}\right), \quad r_{3}^{+}=\left(\begin{array}{c}
1 \\
-c \\
-p
\end{array}\right)
$$

so that hyperbolicity corresponds to $\hat{p}_{\tau}-p \hat{p}_{e}<0$. Computing $A_{ \pm}^{0}=\left(\begin{array}{lll}1 & 0 & 0 \\ 0 & 1 & 0 \\ 0 & v & 1\end{array}\right)_{ \pm}$, we obtain

$$
A_{+}^{0} r_{1}^{+}=\left(\begin{array}{c}
1 \\
c \\
v c-p
\end{array}\right), \quad A_{+}^{0} r_{2}^{+}=\left(\begin{array}{c}
-\hat{p}_{e} \\
0 \\
\hat{p}_{\tau}
\end{array}\right), \quad A_{+}^{0} r_{3}^{+}=\left(\begin{array}{c}
1 \\
-c \\
-v c-p
\end{array}\right),
$$

Using 2.12 to compute $[U]=[\tau](1,-\sigma,-p)$, we obtain for a 1-shock

$$
\check{\delta}=[\tau] \operatorname{det}\left(\begin{array}{ccc}
1 & -\hat{p}_{e} & 1 \\
-\sigma & 0 & -c \\
-p & \hat{p}_{\tau} & -v c-p
\end{array}\right),
$$

where all quantities are evaluated at $U_{+}$, or, using $p \hat{p}_{e}-\hat{p}_{\tau}=c^{2}$,

$$
\check{\delta}[\tau]^{-1}=(\sigma-c) c^{2}+\hat{p}_{e} \sigma v c=(\sigma-c) c^{2}+\hat{p}_{e} c[p],
$$

recovering (Lop 1 (noting by homotopy to the small amplitude case that $\check{\delta}>0 \sim$ stability) in the form $\frac{\hat{p}_{e}}{c^{2}}<\frac{-\frac{\sigma}{c}+1}{[p]}$, equivalent to $\left(\overline{L o p}_{1}\right.$ by the relation $\hat{p}_{e}=\frac{\bar{p}_{S}}{\bar{e}_{S}}=\frac{-\bar{e}_{\tau S}}{\bar{e}_{S}}$, where $\bar{p}(\tau, S):=-\bar{e}_{\tau}(\tau, S)$. This yields the alternate forms $\frac{\hat{p}_{e}}{c^{2}}<2[p]$ and $\frac{\hat{p}_{e}}{c^{2}}>1[p]$ of (Strong) and (Weak), convenient for computations in form (2.8) involving only a pressure law $\hat{p}$ and not a complete equation of state. When $\bar{e}_{\tau S}<0$, this is equivalent to Majda's condition 2.14 with $\Gamma=\frac{-\tau \bar{e}_{\tau s}}{\bar{e}_{s}}$ rewritten as $\Gamma=\tau \hat{p}_{e}$.

Remark A.1. As we have seen, the computations carried out here are simpler, if anything, than the computations in entropy coordinates 2.10 . However, what is not clear from this formulation is why the computations simplify so, involving only the quadratic formula for a $3 \times 3$ determinant, a circumstance that originates from the decoupled form of the equations in entropy variables. 


\section{Appendix B. Helmholtz EnERGy AND ASSOCIATED ENERGY FunCtions}

The completion of a pressure law $p=P(T, \tau)$ by a compatible energy law $e=E(T, \tau)$ may be obtained more systematically by referring to a related circle of ideas involving the Helmholtz energy

$$
A:=e-T S,
$$

where $e, T$, and $S$ have their usual meanings. Here, $A$ is the Legendre transform of $e$ with respect to $S, T=e_{S}$; that is, the relation between $e$ and $A$ is analogous to the relation between Hamiltonian and Lagrangian energies in classical mechanics. See [W1] or [MP for background discussion. For a nice dicscussion of the Legendre transform and relations to the principle of least action, see [W2].

The key properties of the Helmoltz energy from our point of view are as follows.

Lemma B.1. Considering $A=\tilde{A}(T, \tau)$, we have

$$
p=-\tilde{A}_{\tau}, \quad S=-\tilde{A}_{T} .
$$

Proof. By direct computation, $\tilde{A}_{T}=\bar{e}_{S}(d S / d T)-T(d S / d T)-S=-S$, verifying the second relation. Similarly,

$$
\tilde{A}_{\tau}=\tilde{e}_{\tau}-T(\partial \tilde{S} / \partial \tau)=\bar{e}_{\tau}+\bar{e}_{S}(\partial \tilde{S} / \partial \tau)-T(\partial \tilde{S} / \partial \tau)=\bar{e}_{\tau},
$$

verifying the first.

Evidently, using (B.2), we may recover $A$ from the pressure law $p=\tilde{p}(T, \tau)$, up to an arbitrary function of $T$, by antidifferentiation in $\tau$. More fundamentally

$$
A=-R T \ln \tilde{Q}
$$

where $\tilde{Q}(T, \tau)$ is the (rescaled) partition function coming from the statistical thermodynamic derivation of the equation of state.

Exactness. To check whether a pair of gas laws $e=\tilde{e}(T, \tau)$ and $p=\tilde{p}(T, \tau)$ are "exact," that is, originate from a complete equation of state $A=\tilde{A}(T, \tau)$, or, equivalently, $e=\bar{e}(\tau, S)$, we may note that differentiating $S=\frac{e-A}{T}$ with respect to $\tau$ yields $S_{\tau}=\frac{e_{\tau}+p}{T}$, hence exactness, $S_{\tau}=p_{T}$, is equivalent to

$$
T p_{T}=e_{\tau}+p
$$

We can use this to determine also exactness of a pair of gas laws in the alternative form $p=\hat{p}(\tau, e)$, $T=\hat{T}(\tau, e)$, using the Implicit Function Theorem to rewrite (B.4) as

$$
T \hat{p}_{e}=p \hat{T}_{e}-\hat{T}_{\tau} .
$$

(Here, we are implicitly assuming $\hat{T}_{e}>0$, so that we may invert $T=\hat{T}(e, \tau)$ to solve for $e=\tilde{e}(T, \tau)$.)

Hyperbolicity and convexity. The hyperbolicity condition may be deduced using $\bar{p}(S, \tau)=$ $\tilde{p}\left(\tau, e_{S}(S, \tau)\right)$ to obtain $\bar{p}_{\tau}=\tilde{p}_{\tau}+\tilde{p}_{T} \bar{e}_{S \tau}=\tilde{p}_{\tau}-\tilde{p}_{T} \bar{p}_{S}=\tilde{p}_{\tau}-\tilde{p}_{T} \tilde{p}_{T}(\partial T / \partial S)$, or, by $S=-\tilde{A}_{T}$, hence $\partial S / \partial T=-\tilde{A}_{T T}$,

$$
\bar{p}_{\tau}=\tilde{p}_{\tau}+\frac{\tilde{p}_{T} \tilde{p}_{T}}{\tilde{A}_{T T}}=\frac{\tilde{A}_{T \tau}^{2}-\tilde{A}_{T T} \tilde{A}_{\tau \tau}}{\tilde{A}_{T T}} .
$$

Thus, hyperbolicity, $\bar{p}_{\tau}<0$, holds when $\tilde{A}_{T T}>0$ and $\tilde{A}$ is convex as a function of $(\tau, T)$, or else $\tilde{A}_{T T}=-\partial S / \partial T=-1 / \bar{e}_{S S}<0$ (as usual) and $\tilde{A}$ is not concave. Convexity of $\bar{e}$ may be computed similarly to be equivalent to $\tilde{A}_{T T}<0$ and $\bar{e}_{S S} \bar{e} \tau \tau-\bar{e}_{S \tau}^{2}=\frac{\tilde{A}_{\tau \tau}}{\tilde{A}_{T T}}>0$, or $\tilde{A}_{\tau \tau}>0$ and $\tilde{A}_{T T}<0$.

Example B.2 (Ideal gas). Here, we have $A=-\tilde{R} T \ln \tau-C \tilde{R} T$, from which we find as usual $\check{e}(T, \tau)=\frac{3}{2} \tilde{R} T$ (monatomic gas law, $\gamma=5 / 3$.) 
Example B.3 (van der Waals gas). From the reference [W1, we find for $\Gamma=2 / 3$,

$$
A=-\tilde{R} T \ln \left((\tau-\tilde{b}) T^{3 / 2}\right)-\frac{\tilde{a}}{\tau}
$$

where the rescaled partition function $\tilde{Q}(T, \tau)$ satisfies $\ln \tilde{Q}(\tau, T)=\ln (\tau-\tilde{b})+\frac{\tilde{a}}{\tilde{R} \tau T}+C \ln T^{3 / 2}$, $C=$ constant, and $S=-A_{T}=\tilde{R}\left(\ln \left((\tau-\tilde{b}) T^{3 / 2}\right)+\frac{5}{2}\right)$. Thus, to obtain $\check{e}$, we can just compute $\check{e}(T, \tau)=A+T s=\frac{3}{2} \tilde{R} T-\frac{\tilde{a}}{\tau}$.

Example B.4 (Redlich-Kwong gas). Here [W1], $A=-\tilde{R} T\left(\ln (\tau-\tilde{b}) T^{3 / 2}\right)-\tilde{a} T^{-1 / 2} \ln (1+\tilde{b} / \tau)$.

\section{Appendix C. Numerical Evans function protocol}

For completeness, we briefly describe in this appendix the procedure followed in the numerical Evans function studies of part II. For further details, see, e.g., [BHZ1, BHRZ, BLeZ, Z4, Z5].

C.1. Profile and Evans function equations. We first recall the convenient general forms derived in BHLyZ1 for profile and Evans function equations of a general class of systems (5.2) arising in continuum mechanics: specifically, systems for which $B$ has block-diagonal structure

$$
B(w)=\left(\begin{array}{cc}
0 & 0 \\
0 & b(w)
\end{array}\right), \quad b \in \mathbb{R}^{r \times r}, \quad \Re \sigma(b)>0,
$$

and, along the traveling-wave profile $\bar{w}$,

$$
\operatorname{det}\left(d f_{11}^{1}-\sigma d f_{11}^{0}\right)(\bar{w}) \neq 0 \text { (hyperbolic noncharacteristicity). }
$$

Example C.1. Navier-Stokes equations (6.1)-(6.2) have form (5.2), (C.1) with $w=(\tau, v, T)$, $f^{0}(w)=\left(\tau, v, \check{e}(\tau, T)+v^{2} / 2\right)^{T}, f^{1}(w)=(-u, \check{p}(\tau, T), v \check{p}(\tau, T))^{T}$, and $b(w)=\tau^{-1}\left(\begin{array}{cc}\mu & 0 \\ v \mu & \kappa\end{array}\right)$.

Example C.2. Designer system (7.2) has form (5.2), (C.1) with $w=(u, v)^{T}=f^{0}(w), r=n$.

C.1.1. Traveling-wave system. By the block structure assumption (C.1), (5.3) decomposes into

$$
\begin{aligned}
0 & =f_{1}^{1}(\bar{w})-f_{1}^{1}\left(U_{-}\right)-\sigma\left(f_{1}^{0}(\bar{w})-f_{1}^{0}\left(U_{-}\right)\right), \\
b(\bar{w}) \bar{w}_{2}^{\prime} & =\tilde{f}_{2}^{1}(\bar{w})-\tilde{f}_{2}^{1}\left(U_{-}\right)-\sigma\left(f_{2}^{0}(\bar{w})-f_{2}^{0}\left(U_{-}\right)\right),
\end{aligned}
$$

where $f_{k}^{j}$ denotes the $k$ th block of $f^{j}$. By the Implicit Function Theorem, C.2 guarantees that the first equation may be solved, locally, for $\bar{w}_{1}$ as a function of $\bar{w}_{2}$, either analytically (preferable) as done here for gas dynamics or numerically, so that the second equation defines a flow in $\bar{w}_{2}$. Viscous shock profiles are computed using the reduced system (C.3b).

C.1.2. Integrated Evans system. Changing to co-moving coordinates $x \rightarrow x-\sigma t$ in which $\bar{w}$ is a steady solution, we obtain the linearized eigenvalue equation

$$
\lambda A^{0} w+\left(A^{1} w\right)^{\prime}=\left(B w^{\prime}\right)^{\prime},
$$

where

$$
A^{0}:=d f^{0}(\bar{w}), \quad A^{1} w:=d f^{1}(\bar{w}) w-\sigma d f^{0}(\bar{w})-\mathrm{d} B(\bar{w})\left(w, \bar{w}_{x}\right), \quad B:=B(\bar{w}) .
$$

Defining $W^{\prime}:=\bar{A}^{0} U$, we thus have, integrating (C.4),

$$
\begin{aligned}
\lambda W+A^{1}\left(A^{0}\right)^{-1} W^{\prime} & =B\left(\left(A^{0}\right)^{-1} W^{\prime}\right)^{\prime},
\end{aligned}
$$


or, setting $Z:=\left(\begin{array}{c}W \\ \left(0, I_{n-r}\right)\left(\bar{A}^{0}\right)^{-1} W^{\prime}\end{array}\right) \in \mathbb{C}^{n+r}$, and solving for $(I, 0)\left(\bar{A}^{0}\right)^{-1} W^{\prime}$ in the first block of (C.6) using again the assumption (C.2) (see BHLyZ1), the first-order integrated Evans system

$$
Z^{\prime}=\mathbf{A}_{\text {int }} Z, \quad \mathbf{A}_{\text {int }}:=\left(\begin{array}{ccc}
-\lambda A_{11}^{0}\left(A_{11}^{1}\right)^{-1} & 0 & A_{12}^{0}-A_{11}^{0}\left(A_{11}^{1}\right)^{-1} A_{12}^{1} \\
-\lambda A_{21}^{0}\left(A_{11}^{1}\right)^{-1} & 0 & A_{22}^{0}-A_{21}^{0}\left(A_{11}^{1}\right)^{-1} A_{12}^{1} \\
-\lambda b^{-1} A_{21}^{1}\left(A_{11}^{1}\right)^{-1} & \lambda b^{-1} & b^{-1}\left(A_{22}^{1}-A_{21}^{1}\left(A_{11}^{1}\right)^{-1} A_{12}^{1}\right)
\end{array}\right) .
$$

The integrated Evans function $\tilde{D}(\lambda)$ is computed as a Wronskian of (C.7).

C.2. Winding number computations. The Evans function is analytic in the closed right-half plane where, away from $\lambda=0$, zeros of the Evans function match eigenvalues of the system in both location and multiplicity. Thus we can determine spectral stability of the system by numerically computing the winding number of the Evans function on a semicircle $B(0, R) \cap\{\Re(\lambda) \geq 0\}$ in the right-half plane with $R$ chosen large enough to ensure that any possible unstable eigenvalues are inside the semicircle. We determine the value of $R$ either analytically by energy estimates as in $[\mathrm{Br}$ or numerically by convergence to theoretically-predicted high-frequency asymptotics

$$
D(\lambda) \sim C_{1} e^{C_{2} \sqrt{\lambda}}
$$

as in HLyZ1, BLZ, BLeZ, BZ1, BZ2. The former method is carried out on a case-by-case basis, the latter method supported automatically in the MATLAB-based numerical Evans function package STABLAB [BHZ2].

Here, we follow the standard practice of solving, not the eigenvalue equations (C.4), but their integrated form (C.6) [Go, ZH, HuZ1, HLZ, thus removing the zero of $D$ occuring at $\lambda=0$ as a result of translational invariance of the underlying system (5.2) (see (5.4)). That is, we compute the interated Evans function $\tilde{D}$ instead of $D$. This allows us to perform well-conditioned winding number computations directly through $\lambda=0$ throughout most of the computational domain, greatly speeding performance- the exception being at or near the precise parameter values at which stability transitions appear, at which this difficulty is unavoidable.

To locate unstable roots when they occur, we use the method of moments as described in Bro, BJNRZ, BZ1]. computing moments of roots within a contour $\Gamma$ by the generalized winding number computation $\sum_{r_{j} \in \Gamma^{\mathrm{int}}} r_{j}^{l}=\frac{1}{2 \pi i} \oint_{\Gamma} \frac{\lambda^{l} \partial_{\lambda} \tilde{D}(\lambda)}{\tilde{D}(\lambda)} d \lambda$, recovering $r_{j}$ by solution of an resulting polynomial equation, and using a divide-and-conquer strategy to keep the number of roots per subcontour small enough for optimal accuracy vs. speed (in practice, one or two).

Standard practice (see, e.g., [BHRZ, HLZ, BLeZ]), is to approximate the traveling wave profile using MATLAB's boundary-value solver bvp6c [HM], an adaptive Lobatto quadrature scheme, with projective boundary conditions, and on a truncated, finite computational domain $[-L, L]$, where the values of approximate plus and minus spatial infinity $L$ are determined experimentally by the requirement that the absolute error between the computed profile values at $x= \pm L$ be within a prescribed tolerance of the actual endstates $U_{ \pm}$at plus and minus spatial infinity. See [Be1, Be2] for theoretical justification and rigorous error bounds for this approach. We follow this approach for the designer system (7.2). However, for our gas-dynamical computations near the large-amplitude limit $S_{-} \rightarrow-\infty$, this becomes impractical due to stiffness/presence of multiple scales in the travelingwave ODE, and we depart from this method in favor of a simple shooting algorithm with a stiff ODE solver (specifically, the adaptive mesh solver ode15s supported in MATLAB).

We then approximate the Evans function $\tilde{D}$ numerically following the approach described in detail in BHZ1, BLeZ, BLZ]. To preserve analyticity of the Evans function, we use the method of Kato [BHZ1, BrZ, GZ, HuZ1, K, ZZ, Z7] to obtain a holomorphic initializing basis with respect to $\lambda$. To evolve in $x$ the manifolds whose determinant yields the Evans function, we use the polar coordinate ("analytic orthogonalization") method described in [HuZ1. This method evolves an 
orthonormal solution basis along with a complex radial equation that maintains the property of analyticity. See [HuZ1, Z7] for theoretical justification and error bounds. To ensure an accurate winding number count, an adaptive mesh in $\lambda$ is used requiring that the relative error of change in $\tilde{D}$ for each step be less than 0.2 . Recall, by Rouché's Theorem, that if the relative variation of $D$ is less than 1.0, then winding number accuracy is preserved. These routines are supported in STABLAB BHZ2].

For gas-dynamical systems (4.1) and (4.3), the Evans function is poorly conditioned due to multiple scales/stiffness of the eigenvalue ODE. In this case, we find it necessary to use MATLAB's adaptive mesh stiff differential equation solver ode15s both to solve for the profile and to perform the Evans function computations, rather than the Runge-Kutta-Fehlberg method encoded in ode 45 that is typically used in Evans function computations.

C.3. Hardware and computational statistics. In this short section we detail computational statistics for some typical values in the numerical studies reported in previous sections. All numerical computations were performed on either a Mac Pro with 2 Quad-Core Intel Xeon processors with speed $2.26 \mathrm{GHz}$, the super computer Quarry at Indiana University, or a MacBook with an Intel Core 2 Duo processor with speed $2.0 \mathrm{GHz}$.

The Evans function computations were carried out using MATLAB's implicit ODE solver, ode15s, with relative error tolerance set at $1 \mathrm{e}-6$ and absolute error set at 1e- 8 . For most of the parameters in our studies, we used MATLAB's boundary value solver bvp5c with relative error set at 1e-6, absolute error set at 1e-8, and the boundary error set at 1e-6. For the few parameters for which the boundary value solver failed, we used a shooting method as described above, with ode15s.

We now relate computational statistics, run on the MacBook, for each example system. In each case, a minimum of $40 \lambda$ points were used on a semi-circular mesh with radius $R=10$. Additional points were added to the mesh adaptively so that the relative change in consecutive contour points varied by no more than 0.2 . The full contour was constructed by using the conjugate symmetry of the Evans function. For $C=10$ and $S_{-}=-4$ in the global model, it took 23.6 seconds to compute the profile using a boundary value solver with a tanh solution as an initial guess. It took 176 seconds compute the Evans function on 52 points. For $S_{-}=-4$ in the local model it took 62.1 seconds to compute the profile using a boundary value solver and a tanh solution for an initial guess. It took 95.5 seconds to compute the Evans function on 63 points. For $S_{-}=-4$ in the stable model it took 7.98 seconds to solve the profile with a boundary value solver initialized with a tanh solution. It took 28.9 seconds to compute the Evans function on 40 points.

To give some perspective on the computational difficulty of the example models, we include data for the isentropic gas model considered in BHRZ with $\gamma=5 / 3$ and $v_{+}=1 e-4$. The boundary value solver took 0.899 seconds using a tanh solution for an initial guess. The Evans function took 27.7 seconds to compute on 40 points. As the above data indicates, solving the profile in the example gas systems considered here is much more difficult than in isentropic gas. The profile was amenable to solve using a boundary value solver for $S_{-}$as small as $S_{-}=-200$ for the global model with $C=10, S_{-}=-8.1$ for the local model, and $S_{-}=-11.5$ for the stable model. When using continuation to solve, we took steps of 0.1 in $S_{-}$.

We also compare computational performance on the Mac Pro with its 8 cores, which is able to utilize parallel computing. For $C=10$ and $S_{-}=-4$ in the global model, it took 16.9 seconds to compute the profile using a boundary value solver with a tanh solution as an initial guess. It took 28.75 seconds compute the Evans function on 52 points. For $S_{-}=-4$ in the local model it took 37.78 seconds to compute the profile using a boundary value solver and a tanh solution for an initial guess. It took 17.97 seconds to compute the Evans function on 63 points. For $S_{-}=-4$ in the stable model it took 5.63 seconds to solve the profile with a boundary value solver initialized with a tanh solution. It took 6.81 seconds to compute the Evans function on 40 points. For the 
isentropic model with $\gamma=5 / 3$ and $v_{+}=1 e-4$, the boundary value solver took 0.5833 seconds using a tanh solution for an initial guess. The Evans function took 6.0929 seconds to compute on 40 points.

\section{REFERENCES}

[AGJ] J. Alexander, R. Gardner and C.K.R.T. Jones, A topological invariant arising in the analysis of traveling waves, J. Reine Angew. Math. 410 (1990) 167-212.

[BHLyZ1] B. Barker, J. Humpherys, G. Lyng, and K. Zumbrun, Balanced flux formulations and efficient multidimensional Evans function computations for continuum-mechanical equations, in preparation.

[BHLyZ2] B. Barker, J. Humpherys, G. Lyng, and K. Zumbrun, Numerical stability analysis of rNS detonations, in preparation.

[BHRZ] B. Barker, J. Humpherys, , K. Rudd, and K. Zumbrun, Stability of viscous shocks in isentropic gas dynamics, Comm. Math. Phys. 281 (2008), no. 1, 231-249.

[BHZ1] B. Barker, J. Humpherys, and K. Zumbrun, One-dimensional stability of parallel shock layers in isentropic magnetohydrodynamics, J. Diff. Eq. 249 (2010), no. 9, 2175-2213.

[BHZ2] B. Barker, J. Humpherys, and K. Zumbrun. STABLAB: A MATLAB-based numerical library for Evans function computation, Available at: http://impact.byu.edu/stablab/

[BJNRZ] B. Barker, M. Johnson, P. Noble, M. Rodrigues, and K. Zumbrun, Nonlinear modulational stability of periodic traveling-wave solutions of the generalized Kuramoto-Sivashinsky equation, preprint (2012).

[BLZ] B. Barker, O. Lafitte, and K. Zumbrun, Existence and stability of viscous shock profiles for 2-D isentropic MHD with infinite electrical resistivity, Acta Math. Sci. Ser. B Engl. Ed. 30 (2010), no. 2, 447-498.

[BLeZ] B. Barker, M. Lewicka and K. Zumbrun, Existence and stability of viscoelastic shock profiles, Arch. Ration. Mech. Anal. 200 (2011), no. 2, 491-532.

[BZ1] B. Barker and K. Zumbrun, Numerical stability of ZND detonations for Majda's model, preprint (2011).

[BZ2] B. Barker and K. Zumbrun, Numerical stability of ZND detonations, in preparation.

[BE] A.A. Barmin and S.A. Egorushkin, Stability of shock waves, Adv. Mech. 15 (1992) No. 1-2, 3-37.

[Ba] G.K. Batchelor. An introduction to fluid dynamics, Cambridge Mathematical Library. Cambridge University Press, Cambridge, paperback edition, 1999.

[BM] J.W. Bates and D.C. Montgomery, The Dyakov-Kontorovich Instability of Shock Waves in Real Gases, Phys. Rev. Letters 84, No. 6 (2000) 1180-1183.

[BeSZ] M. Beck, B. Sandstede, and K. Zumbrun, Nonlinear stability of time-periodic shock waves, to appear, Arch. Rat. Mechanics and Anal.

[B-G] S. Benzoni-Gavage, Private communication, 2002.

[BS] S. Benzoni-Gavage and D. Serre, Multidimensional hyperbolic partial differential equations. First-order systems and applications, Oxford Mathematical Monographs. The Clarendon Press, Oxford University Press, Oxford, 2007. xxvi+508 pp. ISBN: 978-0-19-921123-4; 0-19-921123-X.

[B] H.A. Bethe, On the theory of shock waves for an arbitrary equation of state, [Rep. No. 545, Serial No. NDRC-B-237, Office Sci. Res. Develop., U. S. Army Ballistic Research Laboratory, Aberdeen Proving Ground, MD, 1942]. Classic papers in shock compression science, 421-492, High-press. Shock Compression Condens. Matter, Springer, New York, 1998,

[Be1] W.-J. Beyn, The numerical computation of connecting orbits in dynamical systems, IMA J. Numer. Analysis 9: 379-405 (1990).

[Be2] W.-J. Beyn, Zur stabilit at von differenenverfahren für systeme linearer gewöhnlicher randwertaufgaben, Numer. Math. 29: 209-226 (1978).

[Bi] S. Bianchini, Private communication, 2002.

[BiB] S. Bianchini and A. Bressan, Vanishing viscosity solutions of nonlinear hyperbolic systems, Ann. of Math. (2) 161 (2005), no. 1, 223-342.

[Br] L. Q. Brin, Numerical testing of the stability of viscous shock waves. Math. Comp. 70 (2001) 235, 10711088 .

[Bri] L.J. Briggs, Maximum Superheating of Water as a Measure of Negative Pressure, J. Appl. Phys. 26, No. 8 (1955) 1001-1003.

[Bro] J. C. Bronski. Semiclassical eigenvalue distribution of the zakharov-shabat eigenvalue problem. Physica D, 97:376397, 1996.

[BrZ] L. Brin and K. Zumbrun, Analytically varying eigenvectors and the stability of viscous shock waves. Seventh Workshop on Partial Differential Equations, Part I (Rio de Janeiro, 2001). Mat. Contemp. 22 (2002), 19-32.

[CDM] F. Cirak, R. Deiterding, and S. P. Mauch, Large-scale fluid-structure interaction simulation of viscoplastic and fracturing thin-shells subjected to shocks and detonations, Comput. \& Structures, 85 (2007) 1049-1065. 
[CF] R. Courant and K.O. Friedrichs, Supersonic flow and shock waves, Springer-Verlag, New York (1976) $\mathrm{xvi}+464 \mathrm{pp}$.

[Dr] L. O. Drury, Numerical solution of Orr-Sommerfeld-type equations, J. Comput. Phys. 37 (1980) $133-139$.

[Er1] J. Erpenbeck, Stability of step shocks. Phys. Fluids 5 (1962) no. 10, 1181-1187.

[Er2] J. Erpenbeck, Reply to Comments by Gardner, Phys. Fluids 6 (1963), p. 1368 (one page).

[Er3] J. Erpenbeck. Stability of idealized one-reaction detonations, Phys. Fluids 7 (1964), p.684-696.

[Fo] G.R. Fowles, On the evolutionary condition for stationary plane waves in inert and reactive substances, in Shock induced transitions and phase structures in general media, 93-110, IMA Vol. Math. Appl., 52, Springer, New York, 1993.

[FP] H. Freistühler and R. Plaza, Normal modes and nonlinear stability behaviour of dynamic phase boundaries in elastic materials, Arch. Ration. Mech. Anal. 186 (2007), no. 1, 1-24.

[FS1] H. Freistühler and P. Szmolyan, Spectral stability of small shock waves, Arch. Ration. Mech. Anal. 164 (2002) 287-309.

[FS2] H. Freistühler and P. Szmolyan, Spectral stability of small-amplitude viscous shock waves in several space dimensions. Arch. Ration. Mech. Anal. 195 (2010), no. 2, 353-373.

[G] C. Gardner, Comment on Stability of step shocks, Phys. Fluids 6, 1366 (1963).

[GZ] R.A. Gardner and K. Zumbrun, The gap lemma and geometric criteria for instability of viscous shock profiles, Comm. Pure Appl. Math. 51 (1998), no. 7, 797-855.

[GS] D.B. Giaiotti and F. Stel, The van der Waals equation of state, PhD Thesis, Environmental Fluid Mechanics, Physics of the Atmosphere, University of Trieste, International Center for Theoretical Physics

[Gi] D. Gilbarg, The existence and limit behavior of the one-dimensional shock layer, Amer. J. Math. 73, (1951). $256-274$.

[Go] J. Goodman, Remarks on the stability of viscous shock waves, in: Viscous profiles and numerical methods for shock waves (Raleigh, NC, 1990), 66-72, SIAM, Philadelphia, PA, (1991).

[Gr] K.N.H. Greenidge, Studies in the Physiology of Forest Trees. I. Physical Factors Affecting the Movement of Moisture, American Journal of Botany Vol. 41, No. 10 (Dec., 1954), pp. 807-811.

[GMWZ1] Gues, O., Metivier, G., Williams, M., and Zumbrun, K., Existence and stability of multidimensional shock fronts in the vanishing viscosity limit, Arch. Ration. Mech. Anal. 175 (2005), no. 2, 151-244.

[GMWZ2] Gues, O., Metivier, G., Williams, M., and Zumbrun, K., Paper 4, Navier-Stokes regularization of multidimensional Euler shocks, Ann. Sci. École Norm. Sup. (4) 39 (2006), no. 1, 75-175.

$[\mathrm{HM}] \quad$ N. Hale and D. R. Moore. A sixth-order extension to the matlab package bvp 4 c of $j$. kierzenka and l. shampine, Technical Report NA-08/04, Oxford University Computing Laboratory, May 2008.

[HVPM] K. K. Haller, Y. Ventikos, D. Poulikakos, and P. Monkewitz, Computational study of high-speed liquid droplet impact, J. Appl. Phys. 92(5):2821-2828, Sept 2002.

[H] W. G. Hoover. Structure of a shock-wave front in a liquid, Phys. Rev. Lett. 42(23):1531-1534, Jun 1979.

[HLZ] J. Humpherys, O. Lafitte, and K. Zumbrun, Stability of viscous shock profiles in the high Mach number limit, Comm. Math. Phys. 293 (2010), no. 1, 1-36.

[HLyZ1] J. Humpherys, G. Lyng, and K. Zumbrun, Spectral stability of ideal gas shock layers, Arch. Ration. Mech. Anal. 194 (2009), no. 3, 1029-1079.

[HLyZ2] Humpherys, J., Lyng, G., and Zumbrun, K., Multidimensional spectral stability of large-amplitude NavierStokes shocks, in preparation.

[HuZ1] J. Humpherys and K. Zumbrun, An efficient shooting algorithm for Evans function calculations in large systems, Phys. D 220 (2006), no. 2, 116-126.

[HuZ2] J. Humpherys and K. Zumbrun, Efficient numerical stability analysis of detonation waves in ZND, to appear, Quarterly Appl. Math.

[HuZ3] J. Humpherys and K. Zumbrun, Spectral stability of small amplitude shock profiles for dissipative symmetric hyperbolic-parabolic systems. Z. Angew. Math. Phys. 53 (2002) 20-34.

[IT] D. Igra and K. Takayama, Experimental and numerical study of the initial stages in the interaction process between a planar shock wave and a water column, Preprint.

[IMR] A. Imre, K. Martinás, L.P.N. Rebelo, Thermodynamics of Negative Pressures in Liquids, Journal of NonEquilibrium Thermodynamics. Volume 23, Issue 4 (2009) 351-375.

[K] T. Kato, Perturbation theory for linear operators, Springer-Verlag, Berlin Heidelberg (1885).

[Kaw] S. Kawashima, Systems of a hyperbolic-parabolic composite type, with applications to the equations of magnetohydrodynamics. thesis, Kyoto University (1983).

[KSh] S. Kawashima and Y. Shizuta, On the normal form of the symmetric hyperbolic-parabolic systems associated with the conservation laws. Tohoku Math. J. 40 (1988) 449-464. 
[L] P.D. Lax, Hyperbolic systems of conservation laws and the mathematical theory of shock waves, Conference Board of the Mathematical Sciences Regional Conference Series in Applied Mathematics, No. 11. Society for Industrial and Applied Mathematics, Philadelphia, Pa., 1973. v+48 pp.

[LV] N. Leger and A. Vasseur, Relative entropy and the stability of shocks and contact discontinuities for systems of conservation laws with non-BV perturbations, Arch. Ration. Mech. Anal. 201 (2011), no. 1, 271-302.

[LS] H. I. Lee and D. S. Stewart. Calculation of linear detonation instability: one-dimensional instability of plane detonation, J. Fluid Mech., 216:103-132, 1990.

[M1] A. Majda, The stability of multi-dimensional shock fronts - a new problem for linear hyperbolic equations. Mem. Amer. Math. Soc. 275 (1983).

[M2] A. Majda, The existence of multi-dimensional shock fronts. Mem. Amer. Math. Soc. 281 (1983).

[M3] A. Majda, Compressible fluid flow and systems of conservation laws in several space variables. SpringerVerlag, New York (1984), viii+ 159 pp.

[MaZ1] C. Mascia and K. Zumbrun, Pointwise Green function bounds for shock profiles of systems with real viscosity. Arch. Ration. Mech. Anal. 169 (2003), no. 3, 177-263.

[MaZ2] C. Mascia and K. Zumbrun, Stability of large-amplitude viscous shock profiles of hyperbolic-parabolic systems, Arch. Ration. Mech. Anal. 172 (2004), no. 1, 93-131.

[MP] R. Menikoff and B. Plohr, The Riemann problem for fluid flow of real materials. Rev. Modern Phys. 61 (1989), no. 1, 75-130.

[MZ1] G.Métivier-K.Zumbrun, Symmetrizers and continuity of stable subspaces for parabolic-hyperbolic boundary value problems, Discrete Contin. Dyn. Syst. 11 (2004), no. 1, 205-220.

[MZ2] Metivier, G. and Zumbrun, K., variable multiplicities, Hyperbolic Boundary Value Problems for Symmetric Systems with Variable Multiplicities, J. Diff. Eq. 211 (2005), no. 1, 61-134.

[Mo] G. Mortimer, Physical Chemistry, 2nd Ed., Academic Press, (2000) 1116 pp.

[PW] R. L. Pego-M.I. Weinstein, Eigenvalues, and instabilities of solitary waves. Philos. Trans. Roy. Soc. London Ser. A 340 (1992), 47-94.

[PZ] Plaza, R. and Zumbrun, K., An Evans function approach to spectral stability of small-amplitude shock profiles, J. Disc. and Cont. Dyn. Sys. 10. (2004), 885-924.

[SS] B. Sandstede and A. Scheel, Hopf bifurcation from viscous shock waves, SIAM J. Math. Anal. 39 (2008) 2033-2052.

[Se1] D. Serre, La transition vers l'instabilité pour les ondes de chocs multi-dimensionnelles, Trans. Amer. Math. Soc. 353 (2001) 5071-5093.

[Se2] D. Serre, Systems of conservation laws. 1. Hyperbolicity, entropies, shock waves, Translated from the 1996 French original by I. N. Sneddon. Cambridge University Press, Cambridge, 1999. xxii+263 pp. ISBN: 0-521-58233-4.

[Se3] D. Serre, Systems of conservation laws. 2. Geometric structures, oscillations, and initial-boundary value problems, Translated from the 1996 French original by I. N. Sneddon. Cambridge University Press, Cambridge, 2000. xii+269 pp. ISBN: 0-521-63330-3.

[S] R.G. Smith, The Riemann problem in gas dynamics, Trans. Amer. Math. Soc. 249 (1979), no. 1, 1-50.

[Sm] J. Smoller, Shock waves and reaction-diffusion equations, Second edition, Grundlehren der Mathematischen Wissenschaften [Fundamental Principles of Mathematical Sciences], 258. Springer-Verlag, New York, 1994. xxiv+632 pp. ISBN: 0-387-94259-9.

[TZ1] B. Texier and K. Zumbrun, Relative Poincaré-Hopf bifurcation and galloping instability of traveling waves, Methods Anal. and Appl. 12 (2005), no. 4, 349-380.

[TZ2] B. Texier and K. Zumbrun, Galloping instability of viscous shock waves, Physica D. 237 (2008) $1553-1601$.

[TZ3] B. Texier and K. Zumbrun, Hopf bifurcation of viscous shock waves in gas dynamics and MHD, Arch. Ration. Mech. Anal. 190 (2008) 107-140.

[TZ4] B. Texier and K. Zumbrun, Transition to longitudinal instability of detonation waves is generically associated with Hopf bifurcation to time-periodic galloping solutions, Comm. Math. Phys. 302 (2011), no. 1, $1-51$.

[T] Y. Trakhinin, A complete 2D stability analysis of fast MHD shocks in an ideal gas, Comm. Math. Phys. 236 (2003), no. 1, 65-92.

[Tr] D.H. Trevena, The stretching and superheating of liquids, Contemporary Physics Volume 17, Issue 2, (1976) 109-126.

[W] H. Weyl, Shock Waves in Arbitrary fluids, Comm. Pure and Appl. Math. 2 (1949).

[W1] Van der Waals gas wiki, http://en.wikipedia.org/wiki/Van der Waals equation.

[W2] Least action wiki, http://bado-shanai.net/map\%20of\%20physics/mopLeastAction.htm

[ZMRS] N. Zhao, A. Mentrelli, T. Ruggeri, and M. Sugiyama, Admissible shock waves and shock-induced phase transitions in a van der Waals fluid, Physics of fluids 23 (2011). 
[ZH] K. Zumbrun and P. Howard, Pointwise semigroup methods and stability of viscous shock waves. Indiana Mathematics Journal V47 (1998), 741-871; Errata, Indiana Univ. Math. J. 51 (2002), no. 4, 1017-1021.

[Z1] K. Zumbrun, Stability of large-amplitude shock waves of compressible Navier-Stokes equations, With an appendix by Helge Kristian Jenssen and Gregory Lyng. Handbook of mathematical fluid dynamics. Vol. III, 311-533, North-Holland, Amsterdam, (2004).

[Z2] K. Zumbrun, Planar stability criteria for viscous shock waves of systems with real viscosity, in Hyperbolic Systems of Balance Laws, CIME School lectures notes, P. Marcati ed., Lecture Note in Mathematics 1911, Springer (2004).

[Z3] K. Zumbrun, Multidimensional stability of planar viscous shock waves, Advances in the theory of shock waves, 307-516, Progr. Nonlinear Differential Equations Appl., 47, Birkhäuser Boston, Boston, MA, 2001.

[Z4] K. Zumbrun, Numerical error analysis for Evans function computations: a numerical gap lemma, centeredcoordinate methods, and the unreasonable effectiveness of continuous pdflorthogonalization, preprint (2009).

[Z5] K. Zumbrun, Stability and dynamics of viscous shock waves. Nonlinear conservation laws and applications, 123-167, IMA Vol. Math. Appl., 153, Springer, New York, (2011).

[Z6] K. Zumbrun, Dynamical stability of phase transitions in the p-system with viscosity-capillarity, SIAM J. Appl. Math. 60 (2000), no. 6, 1913-1924 (electronic).

[ZS] K. Zumbrun and D. Serre, Viscous and inviscid stability of multidimensional planar shock fronts, Indiana Univ. Math. J. 48 (1999) 937-992.

[Z7] K. Zumbrun A local greedy algorithm and higher order exensions for global numerical continuation of analytically varying subspaces. Quart. Appl. Math. 68 (2010), no. 3, 557-561.

INDIANA UNIVERSITY, BLOOMINGTON, IN 47405

E-mail address: bhbarker@gmail.com

Universität Konstanz, 78457 Konstanz, Germany

E-mail address: heinrich.freistuehler@uni-konstanz.de

INDIANA UNIVERSITY, BLOOMINGTON, IN 47405

E-mail address: kzumbrun@indiana.edu 